\title{
Multimodal Imaging based on Vibrational Spectroscopies and Mass Spectrometry Imaging applied to Biological Tissue: A Multiscale and Multi-omics Review.
}

Michael Tuck ${ }^{1 *}$, Landry Blanc ${ }^{1 *}$, Rita Touti ${ }^{1}$, Nathan Heath Patterson ${ }^{2}$, Sebastiaan Van Nuffel ${ }^{3}$, Sandrine Villette $^{1}$, Jean-Christophe Taveau ${ }^{1}$, Andreas Römpp ${ }^{4}$, Alain Brunelle ${ }^{5}$, Sophie Lecomte ${ }^{1}$, Nicolas Desbenoit ${ }^{1 \S}$.

1: Institut de Chimie \& Biologie des Membranes \& des Nano-objets, CBMN UMR 5248, CNRS, Université de Bordeaux, 1 Allée Geoffroy Saint-Hilaire, 33600 Pessac, France.

2: Mass Spectrometry Research Center, Department of Biochemistry, Vanderbilt University, Nashville, Tennessee 37232-8575, United States.

3: Materials Research Institute, The Pennsylvania State University, University Park, PA 16802, USA.

4: Bioanalytical Sciences and Food Analysis, University of Bayreuth, Universitätsstraße 30, 95440 Bayreuth, Germany.

5: Laboratoire d’Archéologie Moléculaire et Structurale, LAMS UMR 8220, CNRS, Sorbonne Université, 4 place Jussieu, 75005 Paris, France.

\section{Table of Content}

Context num

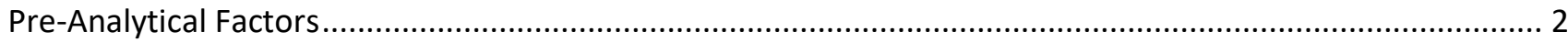

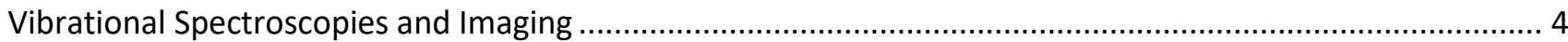

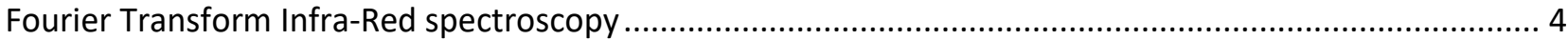

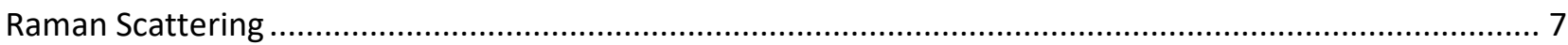

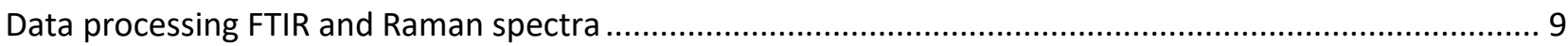

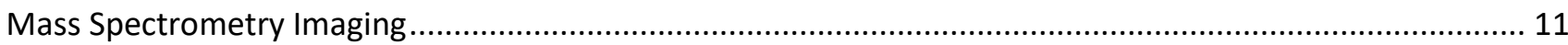

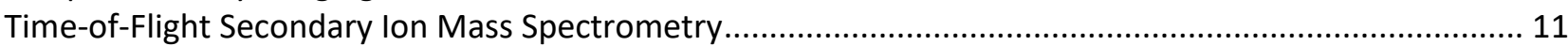

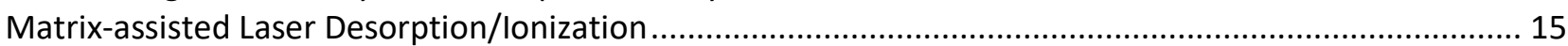

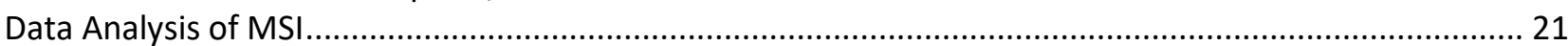

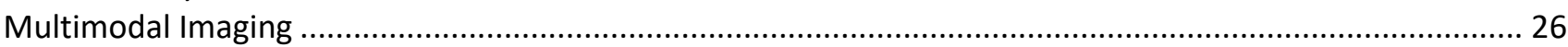

Sample Preparation and Experimental Design for Multimodal Imaging ............................................... 26

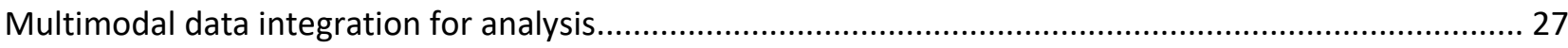

Combining vibrational spectroscopic and mass spectrometry imaging. ................................................. 29

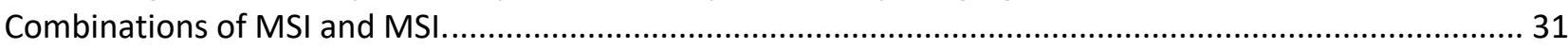

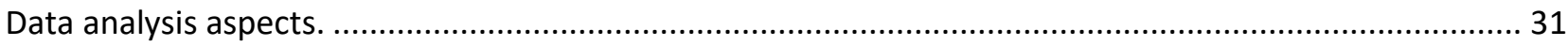

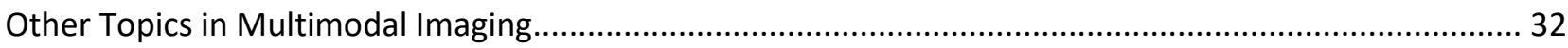

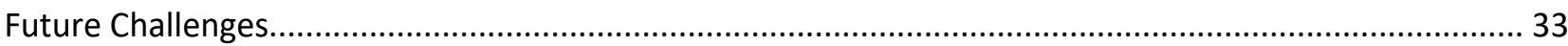

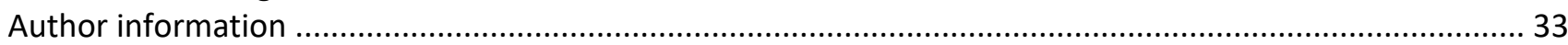

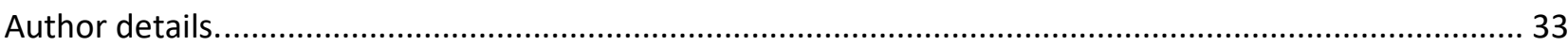

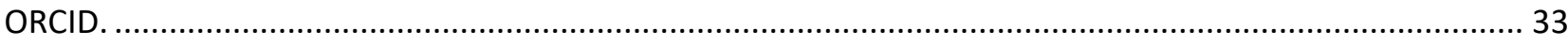

Notes.

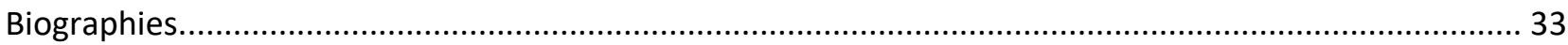

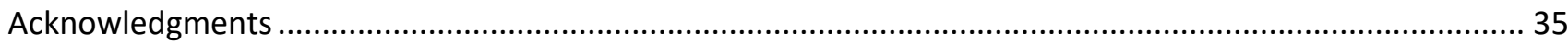

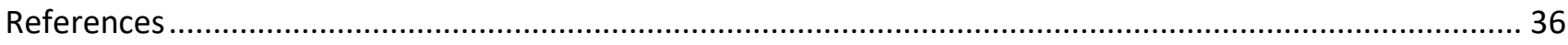




\section{Context}

Molecular imaging has the potential to unearth important spatial and temporal relationships in biological systems, including intercellular signaling as well as environmentally-cued morphological changes. Various chemical imaging techniques show functional groups, molecular weights, etc. but no individual technique to date has the ability to simultaneously access all chemical information. Thus, it is highly attractive to combine information from two or more analytical techniques. Multimodal imaging is a recent, highly effective strategy for acquiring images by combining chemical information from multiplexed platforms. ${ }^{1}$ This emerging integrated imaging approach yields information unattainable from a single method, enabling the evaluation of subtle biochemical changes and opening the way for a quantitative molecular overview of the morphological structure in biological tissues or architecture of cells. As a result, qualitative and quantitative multi-omics investigations have the capability to revolutionize our understanding of disease progression and the healing process. Such a strategy has received increasing attention as it helps to elucidate the complex spatial distribution of biomolecules from the surface of a biological sample while circumventing the specific limitations of an individual imaging technique.

Because multimodal imaging constitutes a vast subject area, emphasis is placed on the well-established vibrational spectroscopies including Fourier transform infra-red (FTIR) ${ }^{2}$ and confocal Raman microscopy (CRM), ${ }^{2}$ as well as mass spectrometry imaging (MSI), also called imaging mass spectrometry, namely time-offlight secondary ion mass spectrometry (ToF-SIMS), ${ }^{3}$ and matrix-assisted laser desorption/ionization (MALDI). ${ }^{4}$ The combination of these label-free and ex-vivo imaging technologies, not fully explored, can be applied either on a single tissue section for direct and unbiased visualization of highly diverse molecular species or in conjunction with a serial section. The strengths and weaknesses of vibrational spectroscopies and MSI, as well as key similarities in workflow, make them highly complementary when gathered in a multimodal imaging approach. Consequently, by adding the high spatial resolution of vibrational spectroscopies with the chemical specificity and sensitivity of $\mathrm{MSI}$, this emerging strategy of multimodal imaging provides new perspectives to decipher the molecular complexity in any biological sample. Based on publication numbers in PubMed, (Figure 1) shows the abundance of different molecular imaging modalities and their evolution over time using keywords related to vibrational spectroscopy, mass spectrometry, their imaging component as well as their corresponding multimodality. Interestingly the trend highlights a constant growth of multimodal imagingrelated articles since the 2010s. As a nascent strategy, and because of the aforementioned benefits, multimodal imaging, independent of the choice of modalities, has the potential to become an increasingly standard next generation analytical approach in the near future.

This review is meant as an introduction for experts in MSI to become acquainted with vibrational spectroscopies and vice versa. This is an entry point for those looking to integrate their analyses in a multimodal fashion and to see how others in the field approach this topic. Although applicable to nearly all ex vivo chemical imaging modalities, this review will focus on combinations of FTIR and Raman spectroscopy with MSI, specifically MALDI and ToF-SIMS. For this purpose, the technologies previously mentioned will be described individually to build a bridge between the sometimes disparate scientific communities. The technological descriptions will help each community understand and assimilate key concepts, foundational knowledge and potential benefits to proper implementation of a multimodal imaging strategy. General topics will be covered from sample preparation to data processing including the latest methodological and technological advances specific to each community. To derive the full benefits of the approach, this review will be illustrated by references focusing on biological and biomedical investigations.

\section{Pre-Analytical Factors}

The tissue sample preparations of various forms of ex vivo molecular imaging techniques share some common points. Biological samples should be harvested with care as to not alter their molecular integrity and with the consideration of all possible future imaging and -omic experiments. This typically involves harvesting tissue, usually from sacrificed animal models or human surgical resections, and quickly flash-freezing to minimize tissue degradation. This can be done by either floating on liquid $N_{2}$, isopentane or gradually freezing with dry ice as to not shatter the tissue. Once frozen, tissues are generally considered acceptable for long-term storage 
in $-80^{\circ} \mathrm{C}$ freezers, although there are caveats to that should be understood. ${ }^{5}$ These fresh frozen (FF) samples are ideal for MSI experiments, vibrational spectroscopies, and are acceptable for histological imaging and pathology, although their storage in expensive freezers can be cost-prohibitive.

Another way of preparing tissue samples is to fix them in formalin to halt protein degradation, and embed them in wax to aid in microtome sectioning in a process known as formalin-fixed paraffin embedding (FFPE). During formalin fixation, reactions causing a web of stable methylene bridges among peptides and proteins occur. ${ }^{6}$ This technique has been popular among histologists for nearly a century; not only does it maintain the spatial integrity of molecules and facilitates sectioning, it also allows indefinite storage at room temperature, providing an economic incentive. Given the benefits and long practice of FFPE, there are hundreds of thousands of samples available, complete with clinical outcomes, even for rare diseases. The downside being that in their current state, they limit the amount of chemical information attainable by $\mathrm{MSI}^{7}$ or vibrational spectroscopy. ${ }^{8}$ Yet, with the allure of accessing libraries of unique samples, efforts have been made to access some of this molecular information with both vibrational spectroscopy and MSI and FFPE samples are widely used for protein, ${ }^{9}$ glycan, ${ }^{10}$ and some lipid ${ }^{11}$ analyses. The process of formalin fixation links proteins and peptides together in a way that they can only partially be reversed. ${ }^{6}$ With $\mathrm{MSI}$, this is typically done with a deparaffinization step followed by a denaturing step known as antigen retrieval. These extreme conditions wash away small molecules and leave mostly large biomolecules in place, the specificities of this which will be described in later sections. In the case of vibrational spectroscopy, paraffin displays strong Raman peaks that usually overlap with the signature of the biological sample. However, paraffin contamination in the vibrational spectrum can be removed by experimental or digital dewaxing approaches. ${ }^{12}$ It is worth noting that dewaxing and fixation steps have been shown to affect lipid analyses and caution should be taken when drawing conclusions based on lipid alterations in FFPE tissue.

The substrate on which the sample will be affixed should be considered carefully as different modalities may require different conductivity and opacity. Important properties to consider when selecting a substrate are the material, spectral background signal and cost. Calcium fluoride $\left(\mathrm{CaF}_{2}\right)$, quartz and fused silica slides are common Raman substrates as they display minimal background interference. However, conventional CRM with high magnification objectives and small pinholes allow for imaging without glass signal contamination, even when imaging a monolayer of cells. ${ }^{13}$ For FTIR, 1-10 $\mu \mathrm{m}$ thick tissue sections are deposited on infrared (IR) transparency windows, usually $\mathrm{CaF}_{2}$ or barium fluoride $\left(\mathrm{BaF}_{2}\right)$ while zinc selenide $(\mathrm{ZnSe})$ can also be used. In MSI, both FF and FFPE, samples are thinly sectioned $(5-20 \mu \mathrm{m})$ with either a cryo-microtome (FF) or microtome (FFPE) and affixed to a substrate. Glass, metal plate or indium-tin oxide coated (ITO) glass slides are utilized in MALDI and ToF-SIMS. It is common for the latter to employ silicon wafers as well. Some tissues do not readily section and a support can help, thus it is common to embed samples prior to sectioning in media such as gelatin or nitrocellulose. ${ }^{14}$ Optimal cutting temperature compound (OCT), popular among histologists, is ill-advised for embedding as the polymer contaminates MS spectra. Once sectioned and mounted on an appropriate substrate, tissues are dried on the bench or in a desiccator box where they await analysis. For Raman, where samples can be much thicker than MSI, relative flatness should be considered as the technique relies on maintaining a consistent focus through the acquisition. Sample flattening is typically achieved with a cryo-microtome or vibratome. Note that before any acquisitions, and independent of the modality, dehydration of tissue sections is required to remove excess water. This is of particular importance when working in FTIR transmittance mode, because water has a broad and intense absorption band at $3400 \mathrm{~cm}^{-1}$. In attenuated total reflection (ATR) FTIR mode, no sample dehydration or slicing should be needed since the signal will not be hampered by water and is depth limited by the technique itself. However, the sample surface properties strongly affect the measured spectra and a smooth, flat surface is suitable to prevent artifacts ${ }^{2}$ and for recognition of structures in visible light. ${ }^{15}$

As an essential step in the workflow, it is useful to have a histological stain to accompany an ex vivo chemical image as it provides a classical, established means to interrogate the morphology of tissue that can orient the analyst or reach a wider biological/clinical community. This can be done after chemical analysis, given that the tissue remains intact and is affixed to a transparent substrate. If not, a serial section can supplement enough morphological information to provide context to the accompanying molecular image. Common stains include hematoxylin and eosin (H\&E) for general purpose staining, periodic-acid Schiff (PAS) used for carbohydrates 
and collagen, and Oil Red $\mathrm{O}$ which stains for fats, among many others. For a recent review, see Clark et Torbenson ${ }^{16}$ and for a one-to-one comparison of H\&E to Raman scattering see Sarri et al. ${ }^{17}$

These pre-analytical sample preparation steps are important to take in to consideration. Proper sample preparation and planning can ensure samples are amendable to multimodal and multi-omic studies that are critical in today's biomedical research. While some molecular imaging modalities are naturally synergistic, others require careful planning and deliberate sample preparation and analysis to effectively combine.

\section{Vibrational Spectroscopies and Imaging}

The vibrational spectroscopic imaging techniques are a label-free methodology that allows the assessment of the distribution of molecular species within a sample. Every molecule is characterized by an intrinsic spectral signature of vibrational modes which can be used to identify, locate and partially quantify specific chemical bonds. There are two major vibrational spectroscopic imaging methods based on two different phenomena: light absorption for FTIR spectroscopy and light scattering for Raman spectroscopy. Both imaging techniques have been shown to be powerful analytical tools in the study of biological materials (cells or tissues). ${ }^{18}$ Raman and FTIR spectroscopies are complementary techniques. Although some vibrational modes may be active in both Raman and IR spectroscopies, these signals arise from different physical processes and have different selection rules. The IR and Raman selection rules are well-known and detailed in the reference book edited by T. M. Theophanides. ${ }^{19}$

\section{Fourier Transform Infra-Red spectroscopy}

Basis of IR signal. The underlying principle of IR spectroscopy is the absorption of photons by a molecule, i.e. the appearance of a band in the IR spectrum results from an act of photon absorption. A photon will be absorbed if its energy matches the difference between the molecule's vibrational energy levels, causing the molecule's excitation. A band is observed on the IR spectrum if the molecular vibration modifies the molecular electric moment. ${ }^{19}$ IR spectroscopy can be separated in two ranges: mid-infrared (MIR) $\left(400-4000 \mathrm{~cm}^{-1}\right)$ and near-infrared (NIR) (4000-12500 $\mathrm{cm}^{-1}$ ) and both can provide specific information about molecular vibrations within functional groups. This review will focus specifically on the "classical" MIR range where the bands are more intense and separated, whereas in NIR they are typically weaker, broader, and overlapping, corresponding to overtones and combinations of fundamental vibrations seen in the MIR. More information about NIR spectroscopy can be found in a recent review. ${ }^{20}$

Assignment of bands observed on FTIR spectrum. One can distinguish three main regions in a MIR spectrum of biological samples: 1) the $800-1800 \mathrm{~cm}^{-1}$ called "fingerprint" region with specific bands of amide bands from proteins, carboxyl groups from of phospholipids and bending vibrational modes of $\mathrm{CH}_{2}$ lipid chains; 2 ) a region free of any biological signatures called the "silent" region at $1800-2700 \mathrm{~cm}^{-1}$; and 3) the $2700-3800$ $\mathrm{cm}^{-1}$ where information comes from stretching vibrations of $\mathrm{CH}$ bonds. In most studies, the entire spectrum is processed for analysis of chemical changes and alterations in tissue morphology. However, since bending and stretching modes originate from the same chemical group, information from those regions can be redundant. Analysis of the fingerprint region alone has been shown to be accurate in several studies. ${ }^{21}$ Despite broader and overlapping bands, the high wavenumber region can also be of interest as it allows for the use of normal glass slides prevented in the fingerprint region because of strong glass absorption. ${ }^{22}$ It has been shown by Mittal et al. that this region can provide accurate histopathology classification of prostate tissues. ${ }^{23}$ In MIR spectroscopy, the wavenumber of the absorption bands can be reliably assigned to the specific chemical functional groups, and the integrated absorbance of a peak is related to the concentration of a molecular component for absorbance mode. This enables identification and quantification of the chemical compounds present in the sample. ${ }^{24}$ In the field of tissue analysis, peptides/proteins, lipids, carbohydrates, nucleic acids or inorganic substances have been explored. In the fingerprint region, the amide I band of proteins (1600-1700 $\mathrm{cm}^{-1}$ ) is of particular interest as its exact frequency value and spectral shape depends on the strength of the intramolecular hydrogen bonds in the peptide backbone. The secondary structure elements of protein can be inferred from the amide I band analyses. ${ }^{25}$ The amide I band of $\alpha$-helices and parallel $\beta$-sheets are observed at 
around $1660 \mathrm{~cm}^{-1}$ and $1630 \mathrm{~cm}^{-1}$, respectively. An antiparallel $\beta$-sheets is identified by two bands at 1620 and $1685 \mathrm{~cm}^{-1}$. The amide I band of an intrinsic disorder protein is expected at $1645 \mathrm{~cm}^{-1}$. Turns are observed at around 1670-1675 cm $\mathrm{cm}^{-1}$. Other major bands are the amide II band $\left(1580-1480 \mathrm{~cm}^{-1}\right)$ used to reveal the presence of proteins, DNA $\left(1250-1080 \mathrm{~cm}^{-1}\right)$, asymmetric phosphate stretching vibrations $\left(\mathrm{V}_{\mathrm{as}} \mathrm{PO}_{2}^{-} ; \sim 1225\right.$ $\left.\mathrm{cm}^{-1}\right)$, carbohydrates $\left(\sim 1155 \mathrm{~cm}^{-1}\right)$, and symmetric phosphate stretching vibrations $\left(\mathrm{v}_{\mathrm{s}} \mathrm{PO}_{2}^{-} ; \sim 1080 \mathrm{~cm}^{-1}\right)$. In the vast majority of studies, the bands are not analyzed one by one, except if a molecule has a very distinctive molecular group in the silent domain ( $\mathrm{CO}$ triple bound or $\mathrm{C}^{2} \mathrm{H}$ ). Numerical tools are used to process the information contained in the spectra.

Chrabaszcz et al. applied FTIR spectroscopic imaging to the identification and visualization of early micrometastasis from breast to lung cancer in a murine model. They showed that FTIR imaging allowed for label-free differentiation and visualization of early micrometastasis foci thanks to differences in spectra between healthy and cancerous cells. ${ }^{26}$ In a recent paper, they used unsupervised hierarchical cluster analysis (UHCA) to differentiate tissue cross-sections into four classes assigned to the cell phenotypes and defined biochemical changes due to metastasis in a qualitative and semi-quantitative manner based on spectral features. ${ }^{27}$ The most pronounced spectral changes associated with the extracellular matrix remodeling process were found in the region below $1400 \mathrm{~cm}^{-1}$ where they observed bands attributed to the amide III mode of lung fibrillar proteins and glycoprotein- and collagen-associated carbohydrate moieties. The results suggested that extracellular matrix remodeling with the progress of the metastasis induced a decrease in the total protein content, as well as an increase of fibrillar proteins depending on the cell type. They also detected alterations in the secondary conformations of proteins and changes in the level of hydroxyproline residues and carbohydrate moieties.

Set-up for MIR spectroscopy. MIR spectrometers are built such that a beam from an IR light source is passed through a sample before reaching a detector. The source is usually an inert solid material that when heated electrically yields thermal emission of broadband light (black-body emission spectrum) with a maximum intensity located in IR region. Recently, high flux IR radiation sources have appeared, that may be based on a tunable diode laser, quantum cascade laser (QCL), ${ }^{28}$ or synchrotron..$^{29}$ MIR spectrometers are based on Fourier Transform (FT) devices, leading to so-called FTIR spectroscopy. The sample is irradiated by a broadband source and the spectral response of the sample is obtained through FT of an interferogram, whose every data point is a function of a moving mirror position and contains information about every infrared frequency from the source. This means that all frequencies are being measured simultaneously, resulting in extremely fast acquisitions. Several sampling geometries exist such as transmission, diffuse reflection and ATR. Transmission mode is typically used for acquisition of the spectra of liquid or sufficiently thin solid samples. Because of the high IR absorption of most biological materials, the effective optical path needs to be in the range of micrometers, typically less than $10 \mu \mathrm{m}$ for hydrated samples, to prevent a complete absorption of radiation while for thicker samples only wavenumber regions featuring relatively weak bands may be acquired reliably. This often implies sample preparation, such as sectioning, is required. On the contrary, if the sample thickness and/or absorption is not high enough, transflectance mode may be used, where light goes through the sample twice thanks to a reflective element upon which the sample is deposited. The ATR mode is based on the total internal reflection (TIR) phenomenon: the IR beam enters a high refractive index ATR crystal and reaches the surface at the critical angle between the crystal and sample. It will thus undergo TIR before going through the collection system. The internal reflection creates an evanescent wave that will probe a few micrometers (0.5$5 \mu \mathrm{m}$ depending on the geometry and on the refractive index ratio $\mathrm{C} / \mathrm{S}$ ) beyond the crystal. By pressing the sample in close contact with the crystal, the evanescent wave can be absorbed by the sample and the collected beam will reflect the absorbance. The ATR-FTIR method is particularly relevant for aqueous media since no saturation of the $-\mathrm{OH}$ bands of water is observed, allowing its contribution to be easily subtracted. A detailed description of ATR system can be found in a recent review by Chan et Kazarian. ${ }^{18}$ The IR sensitive detector converts the light reaching its surface into an electrical signal proportional to the light intensity. Most systems use liquid nitrogen cooled highly sensitive Mercury cadmium telluride detector, although thermoelectric cooling may be found in modern spectrometers. 
FTIR imaging. The basis for spatially resolved spectroscopy is termed microspectroscopy, in which an optical microscope is combined with a spectrometer. This enables acquiring an IR spectrum from an extremely small sample area down to a few micrometers of diameter ${ }^{2}$. A three dimensional data set is obtained after IR imaging, as a two dimensions (2D) spatial array in which each pixel contains a full FTIR absorption spectrum. Two experimental modalities exist for creating the hyperspectral dataset for FTIR imaging: point by point mapping and spectroscopic imaging. In point-by-point mapping, a full spectrum is recorded sequentially from every single point of the sample that is placed on a motorized stage. This mode gives access to better signalto-noise ratio and higher spectral resolution, at the detriment of speed. Indeed, the acquisition time for each spectrum can take up to $10 \mathrm{~s}$, so acquiring a full image can be time consuming, especially for a large area. ${ }^{21}$ In spectroscopic imaging mode, FTIR hyperspectral image is recorded at once using a two-dimensional detector, either an FPA (Focal Plane Array) or a CCD (Charged Couple Device) detector. Spectra from thousands of pixels can be recorded simultaneously across the full range of wavelengths leading to full image acquisition in a few seconds. Hence in situ changes can be characterized and a time-resolved evolution of the sample can follow. While most FTIR imaging studies are performed in transmission or transflectance (reflection and transmission) mode on tissue slices with a microscope coupled to an FTIR spectrometer, Kazarian's group has extensively explored the field of ATR-FTIR imaging. In a well-documented review, ${ }^{18}$ they described the concept of the two modalities of micro- and macro- ATR-FTIR, i.e. with or without a microscope for increasing spatial resolution.

FTIR imaging lateral and axial resolution. One drawback of FTIR imaging is the relatively limited spatial resolution compared to that of Raman or fluorescence microscopies. Indeed, optical diffraction rules set a fundamental limit for spatial resolution of $0.61 \lambda /$ NA named Rayleigh criterion, where $\lambda$ is the illuminating wavelength and the numerical aperture (NA) of the optical system, so one cannot expect much better resolution than $\lambda / 2$, i.e. around $10 \mu \mathrm{m}$ in the $400-4000 \mathrm{~cm}^{-1}(2.5-25 \mu \mathrm{m})$ range, ${ }^{30}$ especially in the fingerprint region. When taking into account optical aberrations, actual resolution is even worse, around $\lambda$ to $3 \lambda^{15}$. The highly collimated synchrotron IR beam is much brighter than conventional Globar sources. This allows acquisition of spectra with excellent signal-to-noise ratio (up to 1000 times greater to bench-top acquired spectra) and diffraction-limited spatial resolution $(2.2 \mu \mathrm{m})$ can thus be attained. ${ }^{29}$ Another possibility to improve resolution is via the use of ATR-FTIR imaging. Since the ATR crystal presents a high refractive index (e.g. 4 for Germanium, Ge), the NA of the optical system increases 4 times, which in return improves the spatial resolution by 4 times. In a recent study, Song and Kazarian have studied colon cancer tissues using a large area Ge crystal coupled with $64 \times 64$ pixels FPA detection. The total field of view was $70 \times 70 \mu \mathrm{m}^{2}$ with a resolution around $6 \mu \mathrm{m} .{ }^{31}$ Using ATR mode in combination with synchrotron radiation, the achieved resolution can be such as $3 \mu \mathrm{m}$ as shown by Vongsvivut et al. ${ }^{30}$ The authors were able to image Plasmodium falciparum parasites in single Red Blood Cells. Despite this improved spatial resolution, ATR imaging can lead to tissue damage, because of the high pressure needed to ensure the good contact between crystal and sample. Moreover, depending on the wavenumber, the depth of penetration of the light will be different. ${ }^{15}$ To achieve three dimensional spatial reconstruction of chemical composition in tissue is a difficult task in FTIR imaging. By nature, transmission or reflectance measurements cannot offer axial resolution better than the thickness of the sample, and the relatively poor axial resolution due to the IR light makes three dimensions (3D) imaging quite deficient even in reflectance mode. In ATR-FTIR imaging mode, one can benefit from the variation of the light's penetration depth, leveraging the angle of incidence to reconstruct $3 \mathrm{D}$ images, ${ }^{15}$ but only on a micrometer range.

FTIR imaging new developments. FTIR imaging at the tissue scale is a relatively mature technique with few breakthrough developments occurring in the last years. Two major axes of further improvement currently investigated are improving the lateral resolution to the nanoscale and decreasing the acquisition time to make the technique clinically suitable. ${ }^{32}$ Atomic force microscopy-based infrared spectroscopy (AFM-IR) is an emerging technique that combines the nanometric resolution of the AFM tip with the identification of functional groups from IR spectroscopy. ${ }^{33}$ In a few words, the tip of an atomic force microscope will locally detect the thermal expansion of a sample resulting from local absorption of IR radiation. This technique has been successfully used for studying bone, ${ }^{34}$ skin, ${ }^{35}$ and hair. ${ }^{36}$ 
In the road to bringing IR imaging to the clinic, it is necessary to improve the speed of data acquisition to allow for imaging of whole biopsies at a rapid rate. ${ }^{32}$ The acquisition of a limited spectral range via a discrete subset of spectral positions, typically less than 30 bands, can often be used to reduce data acquisition time while maintaining the analytical capabilities of IR spectroscopy and imaging. ${ }^{37}$ Mohamed et al. showed that 2D covariance analysis using 4 wavenumbers can identify the 4 main cell types present in breast cancer tissue sections. ${ }^{38}$ They could accurately classify tissue sections recorded by transflectance in the presence of paraffin while the training set was obtained on dewaxed tissue sections by transmission, even when the test set was collected with a different brand of FTIR microscope and a different pixel size. Despite the different recording conditions, separating extracellular matrix from carcinoma spectra was successful, underlying the robustness of this univariate model. These results are very promising for the possibility to achieve very fast data acquisition on $\mathrm{QCL}$ infrared imaging microscopes.

While not compatible with FT technology, this idea has led to the development of discrete frequency infrared microscopy (DF-IR) systems, predominantly using QCL, especially from the Bhargava and Petrich groups with in-house developed QCL IR imaging systems. ${ }^{39}$ In DF-IR, high signal-to-noise ratios can be achieved rapidly by averaging a small spectral range with 100 -fold gains in speed reported. ${ }^{40}$ The very high intensity and directionality of QCL compared to Globar sources make it possible to use uncooled arrays for the detection, which is a particular interest since they are much cheaper and available in much larger formats, improving field of view and time of acquisition. QCL has other advantages such as ease of fabrication, robustness and facilitate polarization studies. ${ }^{41} \mathrm{~A}$ QCL provides tunability over hundreds of $\mathrm{cm}^{-1}$ and commercially available systems consist of multiple modules, to cover the full fingerprint region.

QCLs are not limited to distinct wavenumbers studies but can be used on larger continuous wavenumber regions. Kuepper et al. has presented a feasibility study using a QCL-based infrared microscope with a $480 \times$ 480 pixel FPA detector for rapid and label-free classification of colorectal cancer tissues that provide infrared images with a measuring time of $47 \mathrm{~s}$ for a spectral hypercube of $2 \times 2 \mathrm{~mm}$ field of view with $2 \mathrm{~cm}^{-1}$ resolution from 1800-948 cm $\mathrm{cm}^{-1}{ }^{21}$ They analyzed samples from 110 patients with UICC-Stage II and III colorectal cancer, showing $96 \%$ sensitivity and $100 \%$ specificity as compared to histopathology, the gold standard in routine clinical diagnostics. They successfully reduced the measuring time fitting the pathological workflow with lowest possible intra- and inter-operator variability.

\section{Raman Scattering}

Raman spectroscopy. When a molecule scatters light, most of the photons are elastically scattered which corresponds to the Rayleigh scattering: the energy of the scattered photons is the same as the incident photons. However, a small fraction of light $\left(1 / 10^{7}\right.$ photon) may be inelastically scattered. Raman spectroscopy uses monochromatic light, often in the NIR, visible or UV range to exploit the phenomenon of inelastic scattering, the Raman effect. It results from the interaction of light with vibrational modes associated with chemical bonds within the sample. The Raman effect describes the excitation of photons to virtual energy states and is characterized by a loss (Stokes) or gain (Anti-Stokes) in energy of the scattered photons. This shift in energy carries information regarding the molecular structure. A Raman spectrum is generally displayed by the wavenumber shift from incident to scattered photons against the scattering intensity, so that the $x$-axis will not depend on the excitation wavelength. Raman spectrum fingerprint and high wavenumber regions are rich in information regarding the characterization of biological samples. Like an FTIR spectrum, a unique Raman spectrum can carry information on the biomolecules that constitute the tissue such as lipids, peptides/proteins, nucleic acids, polymers and carbohydrates. One can obtain information about proteomics (structure and identification of proteins), lipidomic (lipid saturation and composition changes), metabolomic (composition and changes of metabolites) and genomic information from Raman spectroscopy. ${ }^{42}$

Confocal Raman Microscopy. To obtain spatially resolved information, spontaneous Raman spectroscopy is most often coupled with a confocal microscope. Such a setup consists of a laser source, a confocal Raman microscope and a diffractive spectrometer equipped with a thermoelectrically-cooled CCD camera as the detection unit. The spatial resolution of a confocal Raman system is generally diffraction-limited at approximatively $\lambda / 2$ as mentioned in the FTIR section and can thus range between hundreds of nanometers 
(for UV and visible excitation wavelengths with high NA objectives) to micrometers (NIR and low NA objectives). In the confocal setup, a pinhole rejects the signal from the out-of-focus region enabling good axial resolution. Coupled with an automated sample positioner, it is possible to record spectra from any sample spot in 3D and to acquire Raman molecular images. The 3D Raman acquisitions are a major benefit when elucidating the molecular complexity of the chemical species in situ.

One of the major advantages of Raman spectroscopy in biological studies is its ability to record label-free, nondestructive spectral information, as in FTIR, and its excellent compatibility with aqueous environments, in opposition to FTIR. Another important advantage is the quasi absence of sample preparation as described previously. Figure 2 details the workflow for tissue analyses by confocal Raman spectroscopy. The use of confocal Raman for biological applications has recently been reviewed by Gomes da Costa et al. ${ }^{43} \mathrm{CRM}$ is used in many different fields such as drug delivery, ${ }^{44}$ diagnosis of diseases such as cancer ${ }^{45}$ or tissue engineering. ${ }^{46}$ In histopathology, Raman imaging, also called hyperspectral Raman imaging, is a valuable complementary tool to detect, locate and delineate diseased tissues. Raman imaging has shown to improve diagnostic accuracy as it reveals a deep molecular understanding of the biochemical changes in affected tissues in comparison with healthy tissue. For example, Acunto et al. have applied Raman imaging to diagnosing and grading chondrogenic tumors. They have shown a progressive degradation of collagen type II, calcification and cellular proliferation in tissues ranging from enchondroma to chondroscarcoma by Raman imaging using unsupervised and supervised statistical tools like principal component analysis (PCA) and linear discriminant analysis (LDA), to be detailed below. By combining histological and Raman spectroscopy results, they provided a comprehensive grading of malignant cartilaginous tumors. ${ }^{47}$ Kopec et al. has shown significant alteration of the chemical composition around blood vessels in the tumor mass of human breast tissue by Raman imaging. ${ }^{45}$ In breast cancer, the development of new blood vessels called angiogenesis is a critical step in cancer's growth, progression and metastasis. The results have shown the importance of the network of collagen, fibroblasts and glycocalyx matrix formed around the blood vessel. Raman spectroscopy is capable of identifying type III collagen fibrils without PAS staining. Thanks to the high spatial and spectral resolution provided by Raman imaging, the authors were able to demonstrate the location and distribution of the epithelial glycocalyx. Also, they have shown that oncogenically transformed cells exhibit enhanced lactic acid, and glycogen activity that can be easily monitored by Raman imaging. ${ }^{45}$ To enhance spectral quality, Raman imaging may require long acquisition times. Many efforts were deployed by researchers to improve data acquisition rate for spontaneous Raman tools such as CRM. ${ }^{48}$

Advanced Raman technologies. Compressive Raman Technology (CRT) is a major innovation in this field. This technology selects only interesting frequency regions when recording a hyperspectral image instead of acquiring a complete vibrational Raman spectrum per pixel, which is the case in conventional methods. Scotté et al. compared their CRT build spectrometer with conventional state-of-the-art hyperspectral Raman systems equipped with $C C D$ and EMCCD (Electron Multiplying CCD) detectors. They have demonstrated that CRT with fast scanning scheme is $\times 100$ times faster than the CCD based system and $\times 10$ times faster than the EMCCD based system. A $100 \times 100$ pixels image requires 100 seconds for a CCD system, 10 seconds for EMCCD system and less than 1 second for a CRT system. ${ }^{49}$

It is well known that the efficiency of Raman scattering is very low which means a great number of Raman spectra must be acquired to achieve signal quality. During such long acquisition, degradation of the sample and alteration in molecular distribution may occur. Advanced Raman technologies such as Coherent Raman Spectroscopy (CRS) have emerged to overcome the weaknesses related to spontaneous Raman methodologies in terms of speed of acquisition and intensity of Raman signal. CRS offers greater signal and speed over conventional spontaneous Raman methods. CRS imaging is carried out using high intensity excitation laser, usually NIR, with short pico- to femtosecond pulses which make it a non-destructive technique. There are two main CRS imaging techniques used for tissues imaging: Coherent Anti-Stokes Raman Spectroscopy (CARS) and Stimulated Raman Scattering (SRS) microscopy. The two techniques are comprehensively reviewed elsewhere. ${ }^{50}$ They are complementary processes occurring simultaneously within the sample. These techniques rely on rather complex instrumentation requiring two laser light fields called "pump" and "stokes". The difference of frequency between the two lasers must be tuned to match a molecular vibration or a set of 
molecular vibrations. The frequency difference between the two lasers creates a coherent condition, which result in a significant increase of the Raman signal compared to the conventional spontaneous Raman. The frequency difference must be adjusted sequentially to match each specific molecular vibration, which makes it a slow approach. New advances in fiber technology have emerged allowing faster tuning, up to two orders of magnitude compared to state of the art reference light source, enabling Raman imaging of several bands at high speed and high spatial, spectral selectivity. ${ }^{51}$ Although CARS and SRS present numerous advantages, their resolution is still diffraction limited. New technologies are developing to overcome this issue. For example, Gong et al. recently presented work on saturated Stimulated-Raman-scattering (SSRS) microscopy to break the diffraction limit for far field super resolution imaging in biological systems. ${ }^{52}$ For CARS, Gong et al. reported a higher-order coherent anti-Stokes Raman imaging to break the diffraction limitation. ${ }^{53}$

\section{Data processing FTIR and Raman spectra}

Visualization. Visualization of the information contained in the vibrational 3D dataset call for a reduction in dimensionality to construct a meaningful two-dimensional image. Simple chemical images can be generated by plotting the integrated absorbance of a spectral band but much more advanced analysis of image are used..$^{54,55}$

Pre-processing. The recorded spectra often require processing in order to reduce the effects from undesirable signals such as background signal due to instrumental effects and/or sample preparations. ${ }^{56}$ For background correction, a large variety of algorithms exists belonging - for the majority - to one of these three families: (i) polynomial fitting, (ii) wavelet transformation, and (iii) first- and second-derivatives. Other algorithms based on frequency-domain filtering, PCA, and artificial neural networks (ANN) may also be used. ${ }^{57}$ On the other hand, the noise present in the high frequency components can be reduced by smoothing techniques like Savitzky-Golay filters, ${ }^{58}$ and kernel smoothing based derivatives. Finally, normalization is an important preprocessing step, the aim of which is to homogenize the intensity levels of the spectra in order to subsequently compare them. The simplest methods use the total sum or the min-max intensity values. ${ }^{59}$

Spectral processing. The usual pipeline for processing spectra (as detailed in Butler et $a l .{ }^{60}$ ) is composed of (i) an enhancement step followed by (ii) a processing step using machine learning (ML) techniques in order to classify spectra or compute clusters of similar spectra. In all of these steps, PCA has proven most popular for processing Raman/IR data. Indeed, PCA reduces the spectral information in each pixel by finding the correlated variables as a set of principal components ranked by those that hold the most variability in signal. Thus, it is used for reducing data complexity for interpretation and may be combined with other ML techniques to improve performance.

Univariate analysis. The simplest approach for exploring data is a univariate peak analysis by imaging the intensities of a single Raman peak. ${ }^{44,46,61}$ However, the univariate methodology may, in some cases, lead to limited molecular specificity in complex tissues due to the overlapping of component signatures. To override this limitation, some effort has been made to develop probes for FTIR imaging through the same pathways as used in fluorescence imaging for example. ${ }^{62}$ Those probes fall into several categories. First, one can add a small vibrational tag to a molecule of interest, like an azide bond, that will exhibit a single, narrow and strong IR band in the cell-silent window. Specific probes have also been developed like metal-carbonyl complexes that show intense $\mathrm{CO}$ absorption bands in this same range. ${ }^{63}$ Deuterium-labeled probes have also been developed for in situ tracking of metabolic activities with subcellular resolution in membranes, ${ }^{64}$ cells and multicellular organisms. ${ }^{62}$ Molecules such as alkyne groups, nitriles, conjugated structures and ring structures containing heteroatoms display a unique molecular signature in the silent region between 2000 to $2700 \mathrm{~cm}^{-1}$. It is also possible to use a univariate methodology to image the distribution of a component by means of isotope labeling allowing unique vibrational signatures of components within tissue. The most common isotope labeling is to exchange carbon-hydrogen bonds for carbon-deuterium bonds, which result in a shift in to the silent region. ${ }^{44,65}$ Others labels have recently been developed including the so-called "Carbow", a class of polyyne-based materials allowing multiplexed imaging of numerous labeled molecules by Hu et al. ${ }^{66}$ 
Multivariate analysis and Machine Learning. When univariate tools are not useful, multivariate statistical analysis can be used to extract valuable and accurate information for specific component. Some preliminary steps are required prior to using $\mathrm{ML}$ techniques. First, the input spectroscopy data must be arranged in a matrix. Thus, the Raman/IR data must be converted in order to have one spectrum per row and each column corresponds to a wavenumber, or Raman Shift. The data describe a hyperspace of $\mathrm{N}$ dimensions where $\mathrm{N}$ is the number of wavenumbers, also called features, composing the spectra. Second, a critical problem arising in ML is known as "the curse of dimensionality". In a high-dimensional hyperspace, the data will become sparse and it will be difficult for the ML program to find out similarities (or statistical significance) between the data. Thus, one of the preliminary steps for most ML projects is to reduce the number of features by extracting the most relevant ones. PCA is one of the most popular techniques extracting principal components based on variables in the data. Other techniques like linear discriminant analysis (LDA) and partial least squares (PLS) regression are popular methods for feature selection/extraction (see review of Trevisan et al. ${ }^{67}$ ). For example, Teh et al. ${ }^{68}$ have used a combination of PCA and LDA for identifying dysplasia from gastric mucosa tissue. Thanks to the availability of widely spread open-source ML packages (Scikit-learn, PyTorch, Tensorflow, e1071, etc. in R) and of dedicated Raman/FTIR software (see a software list in Butler et al. ${ }^{60}$ ), these multivariate methods were extensively used to process the Raman/IR data. Some of these techniques require a training phase from a labeled dataset to build a supervised model whereas others are unsupervised methods.

The unsupervised techniques such as k-means and Hierarchical Ascendant Classification (HAC) were used to process the Raman/IR data. HAC creates a hierarchy of clusters from an algorithm and renders its results as a dendrogram. $\mathrm{k}$-means partitions all spectra in to $\mathrm{k}$ clusters (defined by the user) based on similarity derived from distance metrics (e.g. Euclidean distance is the most common but Manhattan, Mahalanobis, Minkowski distances may be used). This iterative algorithm is calculating at each cycle, gravity centers of each cluster and aggregating the closest data to the updated gravity centers. The process stops when there is no longer movement in the clusters. The results are often represented as a segmented image where the spectra belonging to the same clusters are rendered as same colors. This technique can give insights into regions of the image that have similar or different compositions. Gupta et al. used k-means clustering to characterize composition changes in a calcified cartilage and subchondral bone plate ${ }^{61}$ They found tissue-specific changes as a function of histopathological osteoarthritis severity.

In the supervised methods, the first step is to feed the learner with a training dataset (labeled spectra) in order to build the model before using this model to predict labels on new spectra. Among these methods, Raman/IR data have been successfully processed by support vector machines (SVM) ${ }^{69}$ random forest (based on decision trees) ${ }^{70}$ ANN. $^{71}$ More recently, deep learning was applied in FTIR histology by using convolution neural networks (CNN). ${ }^{72}$ Finally, whatever ML method is used, it is important to evaluate the quality of the model. It is often done by calculating the confusion matrix - containing the true/false positives and negatives - used to compute various parameters like the sensitivity, specificity, and/or accuracy. Moreover, a graphical representation of the sensitivity vs. specificity called receiver operator characteristics (ROC) curve may be convenient to visualize the model quality.

Quantification. The non-negative constrained least squares (NCLS) approach is a powerful supervised methodology for the unmixing of spectral data. This technique relies on an imposed constraint to avoid negative abundances of biomolecules. The advantage of this computational analysis remains in the estimation of the contribution of each component in the collected signal from the sample based on a set of spectral references. Thus, it is possible to estimate the abundance fractions of each constituents. ${ }^{73} \mathrm{It}$ is worth noting that the analysis performed on tissue sections remains semi-quantitative because the relation between abundance of a given vibrational band and the true concentrations remains challenging in Raman spectroscopy. Miloudi et al. ${ }^{74}$ used the NCLS approach to estimate the contribution of specific molecules at each point of spectral maps to deliver semi-quantitative images representing the abundance of drug (Delipidol) in skin layers. Another powerful methodology is the multivariate curve resolution (MCR). This statistical tool is applied to deconvolve each Raman pixel into its pure components and associated abundances using the alternating least squares algorithm. ${ }^{75} \mathrm{MCR}$ offers then estimates of the concentrations of 
components in a tissue in contrast to PCA. Albro et al. applied MCR to decompose the fingerprint range spectra to the component spectra of glycosaminoglycan (GAG), collagen, and water, which elucidate the concentration profile of each constituent throughout the tissue. ${ }^{76}$ However, a limitation of PCA is that it does not estimate the concentration of pure components. PCA is often combined with a supervised method like. Acunto et al. used the combination of PCA and LDA to differentiate between tissues, cells and extracellular matrix, and between collagen and non-collagenous components. ${ }^{47}$

\section{Mass Spectrometry Imaging}

In recent years, MSI has risen as a powerful tool to address scientific questions in the biomedical field. The medical and biological disciplines have hitherto relied on microscopy techniques which require fluorescence labeling. Due to spectral overlap, one is usually limited to 3-5 fluorescent labels for a single imaging experiment. MSI, on the other hand, allows for label-free chemical imaging. Moreover, unlike scanning electron microscopy with energy dispersive spectroscopy (SEM-EDS) and Raman microscopy, which respectively provide elemental and chemical bond information, MSI provides more molecular information measuring mass-to-charge ratio $(\mathrm{m} / \mathrm{z})$. In other words, MSI can map the distribution of proteins, carbohydrates, lipids, metabolites and drugs, not simultaneously like vibrational spectroscopy but with high chemically specific, in tissues and cells without antibodies or isotopic tracers.

\section{Time-of-Flight Secondary Ion Mass Spectrometry}

An MSI technique well positioned for cell and tissue analysis is ToF-SIMS because it has the highest spatial resolution of all MSI ionization sources and can detect many biochemical species with high sensitivity. ToFSIMS can detect both inorganic and organic molecules, and it is sensitive enough to perform trace-element analyses. ToF-SIMS is particularly good at imaging lipids, and it also allows high-spatial resolution imaging of nucleic acids, amino acids, metabolites, drugs, toxins, and elements ${ }^{3}$. ToF-SIMS for biological research is a recent development, however. Initially, ToF-SIMS was considered an 'expensive toy for physicists'. From the late 70 s onwards, the technique was heavily used by the semiconductor industry. In the 90s, the technique started being used to investigate organic materials such as plastics. And finally, in the early 2000 s the first ToFSIMS images of biological systems were published thanks to the development of cluster ion beams. Many publications since then have focused on tissue and single cell imaging, but for far too long biological ToF-SIMS research has been merely demonstrative. With the recent technological developments, it is finally time to push for answers to research questions in chemical biology and medicine.

Basic principles. There are several articles and books that describe the principles of ToF-SIMS in detail. ${ }^{77-79}$ However, this review shall briefly describe the workings of a typical ToF-SIMS instrument. Starting with the first half of the acronym: time-of-flight (ToF): a time-of-flight mass analyzer accelerates the ions by applying a static electric field with a potential difference of roughly $2000 \mathrm{~V}$. Both positive and negative ions can be extracted by switching the potential, referred to as positive or negative polarity. This potential energy is converted into kinetic energy and accelerates the ions towards the extractor. After this, the ions drift in a field free tube towards the detector. The velocity of each ion in the drift tube will be dependent on their mass. Lighter ions have higher velocities and arrive at the detector first, whereas heavier ions have lower velocities and arrive at the detector last. This travel time in the order of $\mu$ s is recorded by the detector and can be converted into a mass-to-charge ratio $(\mathrm{m} / \mathrm{z})$ using the following formula: $\frac{m}{z}=2 \mathrm{eV}\left(\frac{\mathrm{t}}{\mathrm{D}}\right)^{2}$, with $\mathrm{m}$ the mass of the ion, $z$ the number of charges of the ion, e the electric charge of an electron, $V$ the acceleration potential, $t$ the flight time and $D$ the length of the flight path. The length of the drift tube is usually about $2 \mathrm{~m}$ long, which means the pressure in our system needs to be low enough to ensure the ions a mean free path of at least that distance. This, and the primary ion gun, is why ToF-SIMS is an ultra-high vacuum technique with main chamber pressures of $10^{-9} \mathrm{mbar}$. The mass resolution will depend on three uncertainties: the time of ion formation, its place in the extraction field and its initial kinetic energy. To reduce the time interval of the ion formation, short ionization pulses are required ( $<1 \mathrm{~ns}$ ). To reduce the uncertainty of the place of ion formation in the extraction field, a flat surface is required. Surface topography has a negative effect on the obtainable mass resolution 
and a flat surface within a few $\mu \mathrm{m}$ is optimal for ToF-SIMS imaging. To reduce the effects of a spread in initial kinetic energy, a high extraction voltage of $2000 \mathrm{~V}$ is applied, and either a reflectron or triple focusing timeof-flight (TRIFT) analyzer is used. A linear ToF only has a mass resolution of 300-400 but a reflectron or TRIFT analyzer can have a mass resolution of 10000 or more.

The second part of the acronym is the ion formation method: secondary ion mass spectrometry (SIMS). It is possible to emit ions from a solid surface using a focused beam of primary ions accelerated with an energy of several keV or tens of keV. Upon impact, a collision cascade takes places which causes molecules of the sample to transition to the gas phase and ionize. SIMS is thus a so-called desorption-ionization method and because primary ions are used to generate ions, the latter are called secondary ions. The emitted secondary ions can be positively or negatively charged and are subsequently extracted to the mass analyzer using a static electric field. Because this focused primary ion beam has a small diameter, it can be rastered over the sample surface to generate an image where every pixel represents an entire mass spectrum. In other words, ToF-SIMS image data is hyperspectral. The ion images showing the intensity of specific ions per pixel effectively visualize the distribution of chemical components at the surface. The spatial or lateral resolution of the 2D images is determined by the diameter of the primary ion beam because the pixel size needs to match the size of the beam spot.

A frequently used primary ion source is the liquid metal ion gun (LMIG) because of its small beam spot and high current. A Bismuth LMIG allows one to select $\mathrm{Bi}^{+}, \mathrm{Bi}_{3}{ }^{+}$and $\mathrm{Bi}_{3}{ }^{2+}$ primary ions. Cluster ions such as $\mathrm{Bi}_{3}{ }^{+}$have a lower energy per incident atom in an ion cluster which limits the depth of the collision cascade and improves density of energy deposited near beneath the surface, and thus the secondary ion emission yield. ${ }^{80}$ The beam diameter of the Bi liquid metal ion gun can be focused down to $150 \mathrm{~nm} \cdot{ }^{81}$ However, because short ionization pulses are required $(<10 \mathrm{~ns})$ for optimal mass resolution, the ion beam is pulsed with deflector plates and often bunched. Bunching means the primary ions in the vicinity of the back-reflector plate are accelerated so they catch up with the other ions arrive at the sample simultaneously. This creates very short, intense pulses but broadens the kinetic energy distribution, leading to a 1-2 $\mu \mathrm{m}$ beam spot. This means that there is a tradeoff between mass and lateral resolution in a typical ToF-SIMS instrument such as the IONTOF ToF-SIMS $V$ or the PHI nanoToF II. The primary ion dose (ions $/ \mathrm{cm}^{2}$ ) is also an important parameter because it leads to two distinct analysis modes: static SIMS and dynamic SIMS. The keV energy of the primary ions is sufficient to break any chemical bond. The collision damage of a keV primary ion is estimated to stretch an area of several $\mathrm{nm}$ around its trajectory and the molecular structures within this volume will no longer match those of the original sample. ${ }^{82}$ By limiting the number of primary ions to avoid hitting the same area more than once, the mass spectra retain molecular information. This is the so-called static limit, which is determined empirically and estimated at $10^{13}$ primary ions $/ \mathrm{cm}^{2}$ for organic samples. In the case of dynamic SIMS, fast erosion of the surface occurs so depth profiling becomes a possibility, but only elemental information is obtained. Not only secondary ions are emitted from the sample surface during the primary ion bombardment, but also an excess of electrons, which causes positive charging of dielectric samples. This charging changes the local extraction voltage, which disrupts the ion extraction. An electron flood gun compensates the positive charging via a beam of low energy electrons during the mass separation when the extraction voltage is absent.

The above describes a pulsed primary ion source instrument. A different instrument design such as the IONOPTIKA J105 does not use a pulsed primary ion source but a direct current primary ion source and pulsed analyzer. ${ }^{83}$ In the pulsed analyzer design the secondary ions pass continuously into the analyzer and are bunched in time and then pulsed into a reflectron analyzer. Consequently, there is no such trade-off between mass and lateral resolution and no dependence on topography or charging.

ToF-SIMS mass spectra are complex but have the following characteristics: at high $m / z$, adduct ions such as $[\mathrm{M}+\mathrm{H}]^{+},[\mathrm{M}+\mathrm{Na}]^{+},[\mathrm{M}+\mathrm{K}]^{+}$, etc. and occasionally $\mathrm{M}^{+}$are detected; a large amount of fragment ions (but depends heavily on the used conditions, e.g. primary ions, energy, sample, etc.); and both organic and inorganic analytes are ionized. These observations can be rationalized as a combination of electron ionization and adduct ionization in the selvedge as described by the desorption-ionization model, put forth by Cooks and Busch. ${ }^{84} \mathrm{~A}$ bottleneck of ToF-SIMS is the low secondary ion yields obtained and a lot of research has been conducted to improve these secondary ion yields. This is also why ToF mass analyzers with high transmission 
are especially suited for static SIMS in addition to being a panoramic technique that detects all chemical signals simultaneously with a wide detectable mass range from 1 to $10 \mathrm{kDa}$.

Sample preparation. An excellent review of the workflow for ToF-SIMS involving biological applications was recently published by Massonnet and Heeren. ${ }^{85}$ The first steps of sample preparation, such as fixation/cryofixation or tissue sectioning, are already described in pre-analytical factors section. However, because the sample preparation is such a crucial step in ToF-SIMS experiments, the following will provide a brief overview and highlight the areas where ToF-SIMS biological sample preparation can differ from that of other techniques.

Due to the ultra-high vacuum, the high water content of cells and tissues complicates the sample preparation of biological materials for ToF-SIMS imaging. Luckily, the field of electron microscopy (EM) has been developing methods to analyze biological samples under high (or even ultra-high) vacuum conditions as far back as the $1950 \mathrm{~s} .{ }^{86}$ However, it is important to note that whereas it is paramount that the sample preparation does not induce significant physical changes to obtain meaningful electron microscopy results, ToF-SIMS has the additional requirement that the sample preparation does not induce significant chemical changes in the sample. There are two ways to introduce biological samples to the vacuum chamber, namely in a dry state or in a frozen state. Most biological sample preparation techniques for ToF-SIMS involve dehydration of the sample, because a dry sample can be kept at room temperature, allowing convenient sample handling and the use of complementary techniques such as optical microscopy or atomic force microscopy prior to ToF-SIMS analysis. Dehydration induces drastic compositional changes in the sample due to the complete removal of volatile components and subjects the samples to mechanical stresses, however. Alternatively, the sample can be imaged in a frozen-hydrated state by performing the ToF-SIMS measurement with a cryogenically cooled stage at a temperature $<-120{ }^{\circ} \mathrm{C}$ below which recrystallization does not occur. ${ }^{87}$ This is referred to as cryoToF-SIMS and is conceptually analogous to cryo-EM. Cryo-ToF-SIMS allows the sample to be measured in as native a state as possible. There is also some evidence that higher secondary ion yields are obtained for higher mass ions, when studying biological samples in a frozen-hydrated state. ${ }^{88}$

A lot of efforts are being made to see if the low secondary ion yield - a major bottleneck of ToF-SIMS imaging - can be improved upon by different sample preparation techniques. One such method is metal assisted (MetA) SIMS, where nanolayers of gold or silver are deposited onto the sample surface. ${ }^{89}$ Some success has also been achieved by matrix enhanced (ME) SIMS using traditional MALDI matrices, ${ }^{90}$ and it has been demonstrated it is even possible to detect intact peptides using ME-SIMS. ${ }^{91}$ Nevertheless, the secondary ion emission only increases effectively in the positive polarity. In addition, it was recently shown that reactive vapor exposure to trifluoracetic acid also improves the secondary ion yield. ${ }^{92}$ Although progress has been made, the precise mechanisms by which MetA-SIMS, ME-SIMS and other sample preparation techniques improve the secondary ion yield are unfortunately not completely understood, and up to now, the use of polyatomic projectiles remains the best way to maximize the secondary ion emission in comparisons to these sample treatments.

\section{Technological advances.}

Dual Beam Depth Profiling. To improve depth profiling and 3D SIMS measurements, it is common to use two separate beams: a high-energy analysis beam and a low-energy sputtering beam. This allows the user to decouple the secondary ion production from the sputtering event. The high-energy analysis beam must have a small spot size and needs to operate below the static limit to avoid too much sample damage. The sputtering beam, however, needs to operate at higher currents but lower energy to avoid subsurface damage. lons such as fullerenes $\left(\mathrm{C}_{60}{ }^{+}\right)$or massive argon clusters $\left(\mathrm{Ar}_{n}{ }^{+}, \mathrm{n}=500\right.$ to 5000$)$ make for more appropriate sputtering beams than monatomic ions, because of their larger size, shallower implantation depth and higher sputter yields causing less residual chemical damage. Over the past 10 years massive argon cluster beams have been shown to outperform $\mathrm{C}_{60}{ }^{+}$beams for depth profiling organics. ${ }^{93}$ Under tightly controlled conditions using an Irganox 1010 model system Seah et al. were able to achieve a depth resolution of 4-5 nm with an argon cluster beam. ${ }^{94}$ Practically, this means that if equipped with a sputter gun such as an Argon gas cluster ion beam, 3D chemical imaging becomes possible with a depth resolution down to $5 \mathrm{~nm}$. This makes ToF-SIMS a chemical 
imaging technique with a lateral resolution comparable to that of a conventional optical microscope and a depth resolution better than can be attained via conventional confocal microscopy. This has allowed for 3D imaging of single cells, ${ }^{95}$ tissues, ${ }^{96,97}$ and biofilms ${ }^{98}$ with high spatial resolution.

Delayed Extraction. Delayed extraction (DE) is a technique that has been used in MALDI-ToF instruments for at least two decades. ${ }^{99}$ DE means the ions are first produced in a weak electrical field and extracted after a predetermined time delay by application of a high voltage pulse. Ions with greater initial kinetic energy will have travelled closer to the extractor than slower ions, which means the slower ions will receive extra potential energy to catch up to the ones with greater initial kinetic energy at the detector. Given the similarities between a ToF-SIMS system and a MALDI-ToF system, it is surprising this capability was not developed until recently. Vanbellingen et al. demonstrated DE in a ToF-SIMS instrument. ${ }^{100}$ In a typical ToF-SIMS instrument there is a trade-off between mass and lateral resolution because the shorter the ion beam pulses for high mass resolution are obtained via bunching which causes the ion beam to broaden. Using $D E$, it is possible to use an unbunched beam for a higher spatial resolution without compromising the mass resolution. This development has allowed the investigation of smaller features in biological materials with a sub- $\mu \mathrm{m}$ analysis beam with reasonable mass resolution. ${ }^{101}$ Recently, it was shown possible to visualize the copper distribution in cells, ${ }^{102}$ and the 3D distribution of $\mathrm{Ag}$ nanoparticles in cells ${ }^{103}$ thanks to the combination of ToF-SIMS and DE.

Tandem MS. One problem of using ToF-SIMS is the ambiguity when assigning a molecule to a certain mass peak. In a typical ToF-SIMS instrument one must rely on the mass and isotopic pattern of an ion and, with some luck, there are positive correlations with lower mass fragments to help identify its structure, which leaves ample ambiguity. A simple and pioneering idea was to capitalize on post-source decay (PSD) as in MALDI, but this method can lead to incomplete and/or mixed fragment ion spectra, and thus was not pursued. ${ }^{104}$ The solution is ToF-SIMS instruments with tandem MS capabilities as was already the case for many MALDI-ToF instruments more than a decade ago. Tandem MS makes it possible to select an ion and send it through a gas collision cell to induce fragmentation of the selected ion. The result is a second mass spectrum of these fragment ions, which constitute a 'pure' mass spectrum of that parent ion if there are no isobaric interferences. Because these ions are generated through collision-induced dissociation (CID) and therefore not subject to matrix effects, it is also possible to use direct spectrum-to-spectrum matching against a reference library of previously observed MS/MS. This is a fairly new development in ToF-SIMS and the three main ToF-SIMS instrument manufacturers - IONOPTIKA, PHI and IONTOF - have gone for different approaches. The first ToF-SIMS instrument to demonstrate tandem MS capabilities was the IONOPTIKA J105 through collision of the secondary ions with (argon) gas in a field free region before the reflectron but after the exit of the buncher that samples the secondary ion stream. ${ }^{105}$ Each precursor and its corresponding product ions continue to travel at the same velocity and are selected using a timed ion gate, i.e. the MS/MS can be considered to be performed in a ToF/ToF configuration. In 2016, PHI built an instrument where a precursor ion of interested can be deflected into a CID cell. ${ }^{106}$ The resulting fragment ions are mass separated in a linear ToF and counted at a second pulse counting detector, producing the MS/MS spectrum. A great advantage here is that MS/MS and traditional ToF-SIMS data are collected simultaneously from the same analytical area in a single experiment, allowing for a high imaging speed and MS/MS imaging. Around the same time, the IONTOF Hybrid SIMS (or OrbiSIMS) was launched, which was developed at the National Physical Laboratory (NPL) in collaboration with IONTOF and Thermo Fisher Scientific as well as experts in drug discovery at GlaxoSmithKline and pharmaceutical science at the University of Nottingham. ${ }^{107}$ This instrument combines a conventional ToFSIMS instrument with an Orbitrap ${ }^{\mathrm{TM}}$, which allows for MS/MS with an incredibly high mass resolution up to 240000 at $\mathrm{m} / \mathrm{z} 200$ and a mass accuracy below $1 \mathrm{ppm}$. This kind of mass resolution and accuracy allows one to determine the chemical formula of each fragment ion in the MS/MS spectrum without any ambiguity. Several of the new ToF-SIMS instruments with tandem MS capabilities have now been in use for a few years and the first publications are starting to emerge. Bruinen et al. mapped the spatial distribution of $\alpha$-tocopherol in 2D and 3D using cultured cells and thin tissue sections using the PHI nanoToF II ToF-SIMS Parallel Imaging $\mathrm{MS} / \mathrm{MS}$ instrument. ${ }^{108} \mathrm{Fu}$ et al. were able to characterize and localize of bioactive $\gamma$-lactones in tropical wood species S. rubra (Figure 3) ${ }^{109}$ Shi et al. used PHI's new instrument to confirm the identity of diacylglycerols and sphingomyelins in arteries undergoing restenosis. ${ }^{110} \mathrm{NPL}$ recently published several papers where they demonstrate the capabilities of the OrbiSIMS instrument. Aoyagi et al. identified that the perialgal vacuoles of 
P. bursaria with symbiotic C. variabilis contains oligosaccharides. ${ }^{111}$ Zhang et al. and Newell et al. also demonstrated the first use of cryo-OrbiSIMS for the 3D molecular imaging of a $P$. aeruginosa biofilm in its native state, ${ }^{112}$ and to localize semi-volatile organic compounds in biological tissues, ${ }^{113}$ respectively. Other groups have also recently published papers that incorporated IONTOF's Hybrid SIMS instrument. Schaepe et al. compared the use of ToF-SIMS, atmospheric pressure scanning microprobe matrix-assisted laser desorption/ionization (AP-SMALDI) Orbitrap MSI, and OrbiSIMS for the chemical imaging of lipids in native human bone sections. ${ }^{114}$ Van Nuffel et al. combined MALDI-ToF, ToF-SIMS, and Hybrid SIMS to identify markers for pulmonary arterial hypertension in human lung tissue ${ }^{115}$.

Quantification. ToF-SIMS is not considered quantitative, especially in the case of molecular imaging below the static limit, due to varying ionization efficiencies which are compound specific and potential non-linearity of the ion signal induced by the aforementioned matrix effect as well as topography, sample charging and detector saturation. Apart from careful sample preparation and mounting, there are technological solutions to the problem of field effects caused by topography and sample charging. For instance, these field effects can be reduced via the use of an extraction delay in instrument designs with pulsed primary ions and the mass spectrometer is expected to be approximately independent of the local environment of the sample in ToFSIMS instruments with a pulsed analyzer design such as the IONOPTIKA J105. Detector saturation can be avoided or at least minimized during data collection and dead time corrections can also be used to reduce non-linearity. A more fundamental issue is the matrix effect, which is still poorly understood and has defied any description beyond phenomenology for many years. Using good reference materials, it is usually possible to produce linear calibration curves, but the fact that the emission probability of any secondary ion in SIMS depends on its environment seriously complicates quantitative analysis in static SIMS. ${ }^{116}$ Very often one must make do with relative measurements when investigating biological samples. By carefully segmenting the ToFSIMS images to ensure ion signals are only selected from similar chemical environments, followed by a normalization step to account for instrumental variation between measurements, it is possible to compare the relative ion intensities for molecular species of interest of different cells and tissues, keeping in mind the very low experimental variability of ToF-SIMS measurements, even on biological samples. ${ }^{117}$ Often ToF-SIMS imaging is used to identify a compound that is more abundant in a tissue or cell and subsequently LC-MS/MS is used to obtain quantitative information regarding that compound. ${ }^{118}$ At this point, it is usually possible to construct a linear calibration curve. It is also possible to obtain relative quantitative information using isotopically labels. For example, to investigate omega-3 and - 6 fatty acids and their lipid turnover in PC12 cell membranes, Philipsen et al. used isotopically labeled substrates to determine relative quantitative information of incorporated products. ${ }^{119}$ Similar approaches can be used when investigating elemental species in biological samples. Again, we are often limited to a comparison of relative ion intensities. ${ }^{120}$ However, Kern et al. were able to obtain a linear calibration curve for $\mathrm{Sr}^{2+}$ in bone by comparing its relative signal intensity in ToF-SIMS against its content determined using inductively coupled plasma optical emission spectrometry. ${ }^{121}$ Finally, the ion implantation method has been used extensively for SIMS quantification of dopants in the semiconductor industry. Smith et al. have demonstrated it is possible to quantify the chlorine containing insecticide permethrin in polymer fibers with ion implanted standards. ${ }^{122}$ It would be interesting if this could be applied to biological matrices.

\section{Matrix-assisted Laser Desorption/Ionization}

MALDI-MSI. While MSI can be performed with many ion sources, MALDI-MSI has been the most popular to date. MALDI-MSI begins by applying a matrix to a substrate, most commonly a 10 to $20 \mu \mathrm{m}$ thick section of biological tissue. MALDI matrices usually share similar properties: a low molecular weight, the ability to receive or donate a proton, conjugated double bonds for a strong optical absorption, and commonly functionalized with polar groups to be used in aqueous solutions. While the exact mechanism of this physical process is still a matter of ongoing research, ${ }^{123}$ matrix is thought to extract analytes from tissue surface, absorb energy from a laser that then desorbs and ionizes analytes in the gas phase (Figure 4). MALDI lasers, such as nitrogen $\left(\mathrm{N}_{2}\right)$ or frequency multiplied neodymium-doped yttrium aluminum garnet (Nd:YAG) lasers typically operate in the 
ultraviolet range but can also be applied in NIR wavelengths with Nd:YAG lasers. In the gas phase, ionized molecules can be introduced to a mass spectrometric analyzer through a vacuum system or an ion guide/funnel. Analytes can then be detected by a specific mass analyzer, such as the rapid ToFs, molecularly specific ion traps/quadrupoles or the high mass resolving power FT based mass analyzers, such as Fouriertransform ion cyclotron resonance (FT-ICR) mass spectrometers or Orbitraps. The laser pulse starts the MALDI event resulting in a mass spectrum that includes ionized small molecules and matrix, metabolites, and up to larger molecules like proteins. By rastering a MALDI laser across the surface of a tissue, collecting spectra at every position (every MSI pixel), one can construct ion maps of $m / z$ values and generate a portrait of thousands of molecules in situ.

While diversity of sample preparation has been an asset to MALDI-MSI, the variance in it, technical intricacies and continuous method development to better target classes of molecules has been a drawback that continues to stifle its progress into the clinic and broader application as well as introduce concerns about reproducibility.

Sample preparation for lipids, metabolites, and drugs. Sample preparation is an essential part of MALDI-MSI. No single method exists to cover all analyte classes and there are always compromises related to the biomolecules to be detected. One of the biggest challenges of MALDI-MSI sample preparation is analyte delocalization and preservation of the in vivo distribution of biomolecules of interest.

After sample collection, the previously described pre-analytical measures are taken to avoid molecular degradation and delocalization. Once the sample is mounted onto a substrate, a specific sample preparation for the MALDI-MSI experiment is performed. The critical choice is the MALDI matrix and its method of application. The matrix must respond to different imperatives: good absorption at the wavelength of the irradiation laser and efficiently induce analyte ionization while limiting fragmentation. The development of new MALDI matrices is still ongoing; a review by Calvano et al. gives an overview of the latest advancements in the development of MALDI matrices for low molecular weight compounds. ${ }^{124}$ According to data from the Metaspace2020 website (https://metaspace2020.eu/datasets/summary?tab=Summary) collecting parameters of more than 4000 MALDI-MSI experiments, the most frequently used MALDI matrices by this community is 2,5-dihydroxybenzoic acid (DHB) and $\alpha$-cyano-4-hydroxycinnamic acid (CHCA) for positive ion mode. These matrices are popular because they allow analysis of a wide range of compounds such as lipids, drugs, or peptides. 9-amino acridine (9AA) and 1,5-diaminonaphthalene (1,5-DAN) are the two most common matrices for negative mode according to Metaspace2020. These matrices have a broad application for low mass molecules, such as metabolites, or lipids. However, the sensitivity for distinct classes of biomolecules can vary when employing different MALDI matrices and some studies have worked to decipher the analyte specificity of commonly applied matrices. ${ }^{125}$

Following matrix choice, its application is paramount and parameters have to be considered such as crystal size, homogeneity of matrix deposition, analyte extraction, and application reproducibility. The size of the matrix crystals (the smaller the better) ${ }^{126}$ and the thickness of the matrix layer on the surface of the sample will have an impact on the lateral resolution and analyte sensitivity. Unfortunately, no universal matrix application exists and those presented below have their strengths and weaknesses. By using a sublimation apparatus one can achieve homogeneous deposition, and fine matrix crystals ${ }^{98}$ thought to be necessary for imaging at high spatial resolutions, at the cost of analyte extraction as there is no solvent interfacing with the surface of the tissue and the matrix. ${ }^{128}$ This process is done by heating matrix under vacuum and chilling the substrate so matrix molecules in the gas phase condense on to the tissue. To overcome the lack of a solvent, a recrystallization step can be performed if necessary. ${ }^{127}$

Matrix can be sprayed on to the tissue as well, either by a painter's airbrush, ${ }^{129}$ home-built apparatus, ${ }^{130,131}$ or commercially-available robotic sprayers like the HTX Sprayer (HTX Technologies, LLC, NC, USA), SunCollect (SunChrome, Friedrichsdorf, Germany) or SMALDIPrep (TransMIT, Giessen, Germany). This technique uses a solvent to apply matrix, which can be optimized to extract specific analytes. Depending on solvent mixture and spray "wetness", molecule extraction is optimized at the risk of delocalization. An appropriate balance needs to be found between too "wet" and too "dry", which may not extract analytes efficiently. 
Reproducibility is a concern for manual spraying, especially from person to person; automatic sprayers fix this issue by controlling variables such as spray distance, nozzle temperature, pressure conditions and substrate temperature and removing the human variable.

Sample preparation for peptides/proteins, glycans, and polysaccharides. Since the completion of the Human Genome Project, focus has shifted toward the proteome as it became clear that these molecules play a more dynamic role in the cell than their DNA coding indicates. With post-translational modifications (PTMs) such as methylation and glycosylation, differential protein abundances and 3D conformations, it became important to gain an understanding of the proteome in situ. This spatial context would yield a better understanding of how proteins were affecting their microenvironment and how that was shaping the physiological processes of the cell. MALDI-MSI is a well-suited analytical technique for delving into such questions yet the physical limitations and nature of large molecules has to be considered.

One way to interrogate the proteome in tissue using MALDI-MSI is to look for endogenous intact proteins. To begin, tissues thaw-mounted on to a substrate have to be washed to remove small molecules such as lipids and endogenous salts. By removing the readily ionizable lipids, proteins no longer have to compete for charge and can be better detected in spectra. Most commonly this is done by washing in Carnoy solution, a mixture of ethanol, chloroform, and acetic acid. ${ }^{127}$ In addition to removing salts and lipids, the tissue is considered fixed, or that the biochemical reactions, including enzymatic activity, have stopped and the molecules are now static.

There are a handful of matrices commonly preferred for intact protein analysis including sinapinic acid (SA) and CHCA and each have their own technical considerations to keep in mind. For instance, 2,5dihydroxyacetophenone (2,5-DHA) has been heralded for its sensitivity and wide coverage of molecular space, however the volatile matrix is not particularly stable under vacuum or at increased temperatures, common ion source conditions for most MALDI-ToF instruments used in high mass range ( $>5000 \mathrm{~m} / \mathrm{z}$ ) proteomic MALDIMSI experiments. This means less time that the matrix would be optimal, or functional, in many MALDI sources. To address this issue, Yang et al. investigated ketone-based derivatives of DHA, synthesizing (E)-4(2,5-dihydroxyphenyl)but-3-en-2-one (2,5-cDHA). ${ }^{132}$ This work demonstrates that improving MALDI matrices is a valuable amelioration of MALDI-MSI and may have a significant impact on protein MSI.

Two factors that make the analysis of large molecules by MALDI-MSI difficult are the lack of a separation technique and the low charge states of ions generated by MALDI ionization. The latter limits the detection of proteins beyond $12 \mathrm{kDa}$ with FT-ICRs and $30 \mathrm{kDa}$ with ToFs. ${ }^{133}$ With most proteins and protein complexes much larger than the mass range of the instrument, techniques are needed to transmit larger ions. Traditionally, ToF instruments have better figures of merit for ion transmission yet recently, Prentice et al. utilized ion funnel technology of a commercial FT-ICR mass spectrometer to improve the mass range while imaging intact proteins. ${ }^{134}$ By adjusting the source region pressure from 2.9 Torr to $750 \mathrm{mTorr}$, they were able to analyze intact protein standards up to $24000 \mathrm{~m} / \mathrm{z}$ and up to $22000 \mathrm{~m} / \mathrm{z}$ on mouse brain and kidney tissue sections, drastically improving the functional mass range for the detection of proteins. This improvement of ion transmission came with caveats that the authors concede are prohibitive. To isotopically resolve the proteins, a transient of 4.47 seconds was needed to acquire $\sim 41,000$ resolving power at $17,000 \mathrm{~m} / \mathrm{z}$ resulting in a 28 hour image acquisition with a $100 \mu \mathrm{m}$ pixel size. To overcome this, the authors took a multimodal approach and performed MSI to H\&E stained microscopy predictive image fusion to be described in the multimodal section. ${ }^{135}$ This resulted in the prediction of ion distributions at $10 \mu \mathrm{m}$ from $100 \mu \mathrm{m}$ MSI image acquisition data with quantifiably good reconstruction scores.

Because MALDI-MSI generates mostly low charge state ions, much of the proteome remains too large to detect with mass spectrometry. To overcome this and elucidate more proteins, researchers can perform an enzymatic digestion to breakdown proteins into their corresponding peptides in what is known as bottom-up or shotgun proteomics. Here, the resulting peptides are analyzed by MALDI-MSI then identified and attributed to the origin protein, which will be discussed in the data analysis section. Peptides are much more amenable to MS and MSI than proteins and bottom-up proteomics is now a well-established technique. ${ }^{136}$ Also important, FFPE tissues can also be utilized with bottom-up MALDI-MSI, as described earlier. Initially, in situ bottom-up proteomics was done in a histology-directed fashion; that is, using a serial section to determine foci or 
histology of interest and analyzing in these spots. This is because enzyme was typically deposited using robotic spotters that limit analyte delocalization. ${ }^{137}$ As informative as this technique is, the field has moved toward spraying trypsin directly on tissue and performing a traditional MALDI-MSI analysis of peptides. ${ }^{138}$

Many bottom-up MALDI-MSI experiments are conducted on ToF-MS instruments because of their high mass range and high throughput, however, they lack the mass resolving power necessary to identify molecules from complex mixtures. Switching to FTMS analyzers provides mass accuracies greater than $3 \mathrm{ppm} .{ }^{139}$ Recently, Huber et al. reported high mass resolving power and high spatial resolution data for the analysis of tryptic peptides using an atmospheric pressure ionization source (AP-SMALDI10, TransMIT, Germany) coupled to an Orbitrap (Thermo Fisher, Germany). ${ }^{139}$ The authors reported the number of peptides detected is similar at 25 $\mu \mathrm{m}$ and $50 \mu \mathrm{m}$ spatial resolution, concluding that the application of the trypsin and ability to identify peptides are the limiting challenges to analyze at higher spatial resolutions but that cellular resolution is in sight. ${ }^{139}$ Trypsin is a commonly used protease as it cleaves a variety of amino acid sites to cover a wide range of chemical space while being selective enough to keep large peptides that can be traced back to their protein of origin. However, there are many proteases that can be employed, capitalizing on their biochemical properties to gain different analyte specificities. Angel et al. recently employed matrix metalloproteinases to analyze elastin and collagen. These ECM proteins have evaded traditional and MS proteomics as they are highly insoluble, have many PTMs and the superstructural organization of the proteins prevents trypsin from reaching cleavage sites. ${ }^{9}$ The authors analyzed FFPE human liver, human heart valves, and porcine heart valves to visualize the in situ distribution of collagen and elastin via COLase-3 and MMP12. By performing bottom-up proteomics with trypsin on serial sections, the ECM proteins were visualized as well as the wide coverage of peptides provided by trypsin. Immunohistochemistry was also performed as an orthogonal validation of the position of elastin and collagen.

$\mathrm{N}$-linked glycans have been another target of bottom-up MALDI-MSI in recent years as post-translational glycosylation has shown to be indicative of tumor growth and cancer progression. ${ }^{140}$ Researchers sought to identify these biomarkers and their localizations in tissue. By using peptide $\mathrm{N}$-glycosidase $\mathrm{F}$ enzyme, researchers were able to cleave glycan biomarkers from their protein carrier and detect them with MALDIMSI in FFPE tissue. ${ }^{10}$ One challenge analyzing glycans is a tendency for in-source decay. Therefore, Holst et al. performed an in situ derivatization of the labile sialic acid group to stabilize the glycan as it underwent mass spectrometric analysis, increasing sensitivity. ${ }^{141}$ Protein glycosylation is involved in many cellular processes and MALDI-MSI has been employed to interrogate other conditions, such as radiation-induced lung injuries, where researchers recently identified changes in $\mathrm{N}$-glycans as a function of time post-exposure localized to specific histology. ${ }^{142}$ In a multimodal approach, Heijs et al. performed PGNase digestion then tryptic digestion on the same tissue section. ${ }^{143}$ There is a concern that the additional sample preparation would cause the delocalization of peptides, however, the researchers demonstrate multiple highly localized peptides. Arnaud et al. took a similar bottom-up approach to enzymatically digest the polysaccharide cell wall of corn stalks by deacetylating with sodium hydroxide and treating with combination of endoxylanase and lichenase. ${ }^{144}$ This work was important in elucidating mechanisms behind the degradation of the cell wall, important for the biofuel industry.

Despite the many studies investigating tissue preparation, ${ }^{145}$ there is no one-size-fits-all approach to the matter and method development is the responsibility of the individual researcher, complicating reproducibility. The multitude of different sample preparations continues to stifle the wider implementation of MALDI-MSI.

Quantitation. MALDI-MSI is primarily used for qualitative analysis because of issues with tissue/matrix heterogeneity, ionic suppression, and analyte extraction disrupt the accurate quantitation of analytes. Over the past decade, researchers have developed methods to minimize these disturbances in order to turn MALDIMSI quantitative (Figure 4). Reviews from Rzagaliski et al. ${ }^{146}$ in 2017, or Tobias \& Hummon ${ }^{147}$ in 2020 have focused on this aspect of MSI. Buchberger et al. ${ }^{4}$ separate quantification in 2 topics: relative quantitation and the emerging absolute quantitation. In most cases, the comparison with established Liquid chromatographymass spectrometry (LC-MS) quantitation obtained from serial sections is still performed. 
Relative quantitation (or semi-quantitation). In relative quantitation, the main idea is to be able to compare as confidently as possible the amount of a given analyte in different area, ${ }^{148}$ or different tissues. Many parameters can disturb analyte signal intensity, such as tissue matrices and local salt concentration, ${ }^{149}$ complicating direct comparisons from different areas. To alleviate this problem, researchers developed different pixel normalization strategies to compensate for signal perturbations. Nearly all MSI software tools include normalization capabilities during pre- or post- data processing. One of the most common pixel normalization strategies is total ion current (TIC), ${ }^{149}$ dividing each peak by the sum intensity of the whole spectra. Still, for a non-targeted approach, another strategy for semi-quantitation is to use a reference peak, typically a matrix peak or a peak from an endogenous molecule known to be homogenous among the tissue. ${ }^{150}$ In this case, internal standards might be used as reference peaks. These standards should be chemically similar to compounds of interest and exogenous to avoid crosstalk with endogenous spectra. ${ }^{151} \mathrm{Hamm}$ et al. demonstrate another approach for exogenous analytes, like drugs, by calculating a tissue extinction coefficient (TEC). ${ }^{152}$ Here, a "pseudo internal standard" (a solution of the targeted analyte), is sprayed on a control section. Then, the TEC of specific tissue areas (e.g. brain white matter vs brain grey matter) is calculated by dividing the average intensity of the analyte signal from the specific tissue area by the average intensity from a reference region (of-tissue). For targeted approaches, the gold standard is the use of a stable isotope-labeled (SIL) analog of analytes of interest ${ }^{153,154}$ applied homogenously on tissue (Figure 4), sometimes during matrix application. This SIL standard retains chemical properties and mimics the ionization efficacy of the analyte of interest but presents as a shifted $\mathrm{m} / \mathrm{z}$ ratio, detectable by MS. Purchasing SIL standards can be expensive.

Absolute quantitation. In order to be able to determine an analyte concentration per volume (or weight) of tissue, the creation of a calibration curve and normalization by internal standards are required. Three calibration curve strategies have been developed by researchers. ${ }^{146}$

The "in-solution" method consists of spotting different concentrations of standard on plate, close to sample section and analyzing. This method is easy to implement and has been used for various analytes, ${ }^{155}$ but does not consider biological matrix effect on extraction yield or ion efficiency and could lead to a misevaluation of analyte concentration. However, combination of the in-solution method with TEC normalization tends to take into account such biological matrix effects. ${ }^{152}$

The "on-tissue" method relies on spotting standard curve directly on a reference tissue. ${ }^{156}$ In case of dosing exogenous analyte (like pharmaceuticals), a standard curve can be made directly with the compound of interest on a control or untreated tissue. In the case of endogenous analytes (lipid or metabolite), standards curves are made with a SIL analog and can be deposit on serial sections. A second SIL standard should be used for normalization. This method takes into account matrix ionization effects and ion suppression but its ability to compensate analyte extraction is still debated. ${ }^{147}$

Finally, the "in tissue" method intends to compensate matrix ionization effects, ion suppression, and analyte extraction by spiking standard curve directly into a mimetic tissue. ${ }^{157}$ In Barry et al., tissue homogenates containing serial concentrations of spiked internal standard(s) are frozen one after the other into a mold. ${ }^{158}$ Then this "standard tissue" can be sectioned and thaw-mounted close to tissue of interest in order to analyze the sample and standard curve together. However, it has been shown that tissue homogenate of a given type of tissue is suitable only for the same type of tissue. Quantitation on different tissue types may therefore not be reliable, likely due to protein binding. ${ }^{159}$ Consequently, quantitative MSI experiments involving different types of tissue (like brain, tumor, or Tuberculosis granuloma) might require as many tissue mimetic models to truly match each region.

Correlation with LC-MS. After a conventional extraction step, LC-MS allows absolute quantitation of targeted analytes at the cost of spatial information. MSI is regularly used in conjunction with LC-MS, on adjacent tissue sections. Once the absolute amount of analyte is determined for a section, an approximate concentration in the MSI section is discerned. This method makes it possible to compare concentration among tissue sections. Absolute quantitation by LC-MS guided by MSI is also a valuable option. The idea is to determine in advance the distribution of target analytes in sample to dissect the regions of interest (ROI), manually at macro-scale or by laser capture microdissection (LCM). MSI and dissection, usually performed on adjacent aligned sections, ${ }^{160}$ can also be performed on the same tissue section. ${ }^{161}$ Liquid extraction surface analysis (LESA), a burgeoning tool for quantitation, will be discussed later in the context of protein identification. 
New technical and methodological processes. One reason for MALDI-MSI's continued popularity over the last $20+$ years is its iterative amelioration. The MALDI-MSI community has built upon itself and borrowed from other scientific disciplines to deliver an ever improving technology. These incremental improvements come in the form of advances in spatial resolution, throughput, and figures of merit such as analyte sensitivity and specificity. Recently, many groups have taken to creative new technical processes to either improve certain facets of MALDI-MSI, like spatial resolution, or to circumvent fundamental obstacles such as the lack of a chemical separation technique.

MALDI sources and mass analyzers. The nascent days of MALDI-MSI, like its predecessor SIMS imaging, relied on existing ToF-MS as these instruments provided quality mass separation with high ion transmission efficiency. ${ }^{162}$ By the late-nineties, commercial MALDI-ToFs could be modified for imaging purposes, resulting in 1-2 minute per pixel manually acquired laser shots. ${ }^{163,164}$ Breakthroughs in spatial resolution came via alterations in laser geometry. ${ }^{165,166}$ Instrumentation companies have been in step with these advancements with most commercially available sources now promising 10 to $20 \mu \mathrm{m}$ lateral spatial resolution routinely, which can often be tuned further to 7 to 2 micron. ${ }^{166,167}$ Cellular and subcellular MALDI-MSI resolution is a milestone that was recognized in the early days of the technique and has been recently realized by several groups. ${ }^{166,168}$ Another improvement in source design is the demonstration of atmospheric pressure (AP) sources being comparable to vacuum ones. ${ }^{169}$ Consequently, AP-MALDI sources can handle volatile compounds/matrix during long image acquisitions. ${ }^{166}$

Of course, ToF based MS lacked the mass accuracy and specificity for informed molecular identifications. MALDI-MSI suffered from a lack of hyphenated techniques like tandem MS (MS/MS). Groups began adding MS/MS capabilities to their imaging. Fourier transform mass spectrometry (FT-MS) combined with MALDI-MSI was an important development as it provided unparalleled mass accuracy and mass resolving power. This allowed for the imaging of complex matrices with the isotopic separation needed to differentiate proteins or to identify lower mass compounds from chemical matrix interference. ${ }^{170}$

Post-Ionization. With questions of ionization efficiency, a technological leap has been achieved with the prospects of post-ionization techniques. The very recent plasma ionization enhancement ${ }^{171}$ demonstrates signal improvements of over three orders of magnitude for some compounds. On other hand, the difficult to overlook MALDI-2 post-ionization technique ${ }^{172}$ is more available now that it is commercially available. ${ }^{173}$ MALDI-2 works by adding a secondary laser perpendicular to the initial MALDI plume event and timing a laser pulse such that any neutrals or molecules in the gas phase but not ionized will become charged and directed towards the mass analyzer. ${ }^{172}$ This combination of MALDI events can increase sensitivity by 100 -fold for some molecular species. ${ }^{172,174}$ Niehaus et al. ${ }^{168}$ and Spivey et al. ${ }^{175}$ applied MALDI-2 to a transmission geometry laser orientation, ${ }^{165}$ which is capable of ultra-high spatial resolutions at the substantial cost of analyte sensitivity. However, with the addition of MALDI-2, analyte sensitivity can be recovered while obtaining spatial resolutions between $600 \mathrm{~nm}$ and $1000 \mathrm{~nm}$.

Some of the lipid classes that benefit most from MALDI-2 are the ones whose sensitivity is affected by ion suppression, like glycerophospholipids. ${ }^{176}$ Concerned with ion suppression effects and misleading MALDI-MSI heat map images, Eiersbrock et al. sought to quantify lipid content by performing MALDI-2-MSI in conjunction with LCM and quantitative nano- high-pressure liquid chromatography (HPLC) Electrospray ionization (ESI) of the same tissue section. ${ }^{177}$ The researchers undersampled while performing MALDI-2-MSI by employing a 30 $\mu \mathrm{m}$ lateral step but a laser ablation size of only $15 \mu \mathrm{m}$. This allowed for the LCM lipid extraction and quantification of areas of white matter and grey matter in mouse brain. With both MALDI and MALDI-2, discrepancies were found between the MSI sensitivity and the lipid content quantified with ESI for nearly all investigated lipid classes. The presence of phospholipids, even in small quantities, was detrimental to glycophosolipids sensitivity in positive mode. It was also observed that local fluctuations in salt composition, such as in the white and grey matter of mammalian brain tissue, was independent from ion species suggesting it is not a main contributor to signal intensity bias. ${ }^{177}$

Ionization efficiency. Some improvement lies with MALDI's low ionization efficiency. Several groups have taken clever approaches to improve the sensitivity of either the entire spectrum, specific analytes or classes of analytes. For instance, chemical derivatization has been applied to various compounds like 
neuropeptides, ${ }^{160}$, neurotransmitters, ${ }^{163}$ metabolites, ${ }^{182}$ etc. This allows molecules to be detected more readily than they would be without such modifications by adding a readily ionizable functional group. ${ }^{183}$ In a different approach, Prentice et al. characterized the FT-ICR method of continuous accumulation of selected ions (CASI) and demonstrated selected ion ejection using a quadrupole mass filter with MALDI-MSI. ${ }^{184}$ These techniques have improved MALDI-MSI by addressing sensitivity and dynamic range. For further reading on improvements in MALDI-MSI as it pertains to sensitivity and specificity, see the recent review by Gilmore et al. $^{78}$

Ion Mobility. Yet, an increase in sensitivity, or mass resolving power, would not alleviate issues of isobaric species in MALDI-MSI. Unlike ex situ MS techniques like LC-MS, MSI lacks a chemical separation event prior to mass spectrometric analysis. Therefore, many groups have begun to incorporate ion mobility MS (IM-MS) to separate species based on their collision cross-section (CCS). ${ }^{173,185}$ There are different technical approaches to performing IM-MS, ${ }^{186}$ but the idea is to pass a packet of ions through a slightly pressurized drift tube and allow air resistance to separate isobaric species based on their CCS, or structural "floppiness". By exiting the drift tube at different times, two species with the same $\mathrm{m} / \mathrm{z}$ can be distinguished. In a recent MSI example involving trapped IM-MS (TIMS), Djambazova et al. showed a $271 \%$ increase in molecular species detected between $\mathrm{m} / \mathrm{z}$ $680-880$ by applying a 650 ms separation. ${ }^{187}$ Of course, this adds another dimension to the already highly dimensional MSI data and tools need to be developed to visualize it. Yet, having two points of data, CCS and $\mathrm{m} / \mathrm{z}$, databases would provide more structural information than MS without IM-MS. ${ }^{185}$ Another interesting challenge as IM-MS becomes more integral to MALDI-MSI is inconsistencies among researchers in how they report their IM-MS data, and even debate over what is actually measured. ${ }^{186} \mathrm{~A}$ multi-institutional guide for researchers reporting IM-MS data was recently written by Gabelica et al. ${ }^{186}$

Recently, the combination of these exciting MSI advancements, IM-MS and MALDI-2, was demonstrated by Soltwisch et al. ${ }^{185}$ They report a $40 \%$ average increase in detectable peaks on rat brain tissue with a signal-tonoise ratio $\geq 20$. To illustrate the advantage of using IM-MS with MALDI-2, a mass window of only 1 Da between $\mathrm{m} / \mathrm{z} 810$ and $\mathrm{m} / \mathrm{z} 811$ detects nine features compared to just five with mass spectrometric analysis alone. The authors note a significant impact on acquisition speeds, however; an IM-MS ramp time of 800 ms decreased data acquisition from 33 pixels/s to just 1.2 pixels/s.

Acquisition speed. Practically, the throughput of these instruments has to be considered. Advancements in stage speed and data acquisition are essential for the broader application of MALDI-MSI and timely analysis is requisite. ${ }^{188}$ Some improvements include increasing the repetition rates up to $10 \mathrm{kHz}$ for Nd:YAG lasers and switching from a discrete pixel mode to continuous raster mode. ${ }^{189}$ With this combination, MALDI-MSI at a rate of 50 pixels/second has been achieved. ${ }^{190}$ Barré et al. recently demonstrated high-speed data acquisition with MALDI-2 post-ionization using a modified source in conjunction with web-based control software allowing for $10 \mathrm{pixel} / \mathrm{second}$ images. Researchers also detail issues with IM-MS drift-time and aliasing effects. In addition, the modified optics allowed for cleaning of the source without venting the instrument, limiting downtime. ${ }^{191}$

The ease in which MALDI-MSI can be amended has kept it relevant in biomedical research, and other analytical fields over the last 25 years. The advances in this technique have made it more alluring to researchers as it approaches its potential as a molecular microscope, articulated in its inception. Also alluring is how MALDIMSI lends itself to multimodal techniques, whether it is imaging with other MSI modalities, other molecular imaging techniques or microscopy.

\section{Data Analysis of MSI}

MSI generates large amounts of multidimensional data (from GB to hundreds of GB) that can be cumbersome for users. A typical ToF-SIMS mass spectrum, for instance, contains around 1000 mass peaks and an average 3D ToF-SIMS image consists of $256 \times 256 \times 256$ voxels, which means the data set is comprised of more than 16 billion data points. The size of the data sets often makes data analysis computationally intractable. Fortunately, there are different analysis methods available to researchers; some are commercial while others are free and open-source (FOSS) or developed in-house. Data processing among the different MSI techniques 
share a common workflow: a signal processing step, data visualization, statistical analysis, molecular identification and validation with an orthogonal technique. ${ }^{192}$ For recent reviews on data analysis pipelines, see Alexandrov, ${ }^{192}$ Verbeeck, ${ }^{193}$ and Buchberger et al. ${ }^{4}$

Preprocessing. Before data analysis, preprocessing can help to reduce experimental variance induced by tissue matrix effect or measurement artifacts. Preprocessing can also be useful to reduce the size of the data in order to be handled more efficiently by computational tools and save processing time. As described by Norris et al., ${ }^{194}$ preprocessing steps such as baseline correction or spectral alignment should be applied to ensure accurate data analysis. For spectral alignment, instead of being performed during preprocessing, another common approach is to use an internal lock mass to perform real-time calibration during acquisition. ${ }^{195}$ This internal lock mass could be a background ion (like a matrix peak) or an exogenous analyte applied with matrix deposition. More recently, Brochu et al., developed a virtual lock masse algorithm for spectral alignment useful for large datasets acquired in single or multiple batches. ${ }^{196}$ Following these preprocessing steps, spectral normalization is performed to compensate pixel-to-pixel peak intensity variations due to environmental and experimental error, ${ }^{197}$ as described in the quantitation section.

Spectral centroiding (often called peak picking) ${ }^{192,198}$ is a method to reduce the spectral dimension and compress data size by a 10x factor. It is commonly performed on-the-fly on FTMS instruments as they can generate large datasets and immediately having a lower dimensional representation is useful. In mass spectrometry, the $m / z$ measurement of the ions will be organized in a peak with a Gaussian shape, or "profile", more or less narrow depending on the mass spectrometer resolution and performance. Centroiding is a procedure to select the central $m / z$ value for each Gaussian peak and to calculate the area under the peak as the centroid intensity. Peaks are turned into a discrete $m / z$ with zero line widths, known as "centroid". With less information describing the signal, the file size will be significantly smaller. Large datasets could still be tedious to handle by computational tools for multivariate analysis, especially for datasets which are too large to be stored on a computer's random access memory (RAM). In that case, one could perform binning, spatially, spectrally or both to reduce the size of the dataset at the loss of spectral/spatial information. However, researchers developed now memory efficient algorithm to avoid to have to discard information from datasets. ${ }^{199}$

imzML - a common data format for MSI data. Standardized data formats greatly facilitate the handling and processing of large datasets. A common standard for mass spectrometry data (mzML) was developed within the framework of the Human Proteome Organization (HUPO). ${ }^{200} \mathrm{~A}$ more specialized data format for MSI has been established within the framework of the EU-funded project COMPUTIS (www.computis.org): the imzML format. ${ }^{201,202}$ All relevant information on imzML including technical specifications, available software tools and implementation examples are provided on the website www.imzml.org.

imzML data is divided into two files. The metadata such is stored in an $\mathrm{xml} \mathrm{file} \mathrm{(*.imzml)} \mathrm{which} \mathrm{is} \mathrm{based} \mathrm{on} \mathrm{the}$ structure of $\mathrm{mzML}$. In addition $\mathrm{mzML}$, the controlled vocabulary of imzML includes imaging-specific information such as number of pixels, $x / y$ image dimensions and stage step size. The mass spectral data of imzML is stored in a separate binary file $\left({ }^{*}\right.$. .ibd) efficiently storing large data sets. Data in the binary ${ }^{*}$.ibd file can be stored in two different ways. 'CONTINOUS' is used for data that share the same $m / z$ axis for all mass spectra, typically the case for unprocessed ToF-MS data. In contrast, peak-picked data and data from FTMS typically have a different $m / z$ array for each spectrum and are stored in the 'PROCESSED' mode. This approach was chosen as it allows for efficient storage of data of varying structure. The two files (*.imzml and *.ibd) are linked by a universal unique identifier (UUID) which ensures that matching files can be distinguished.

imzML data can be obtained by converting proprietary data into imzML with a dedicated converter, e.g. "imzML converter" 203 and the "RAW Converter". ${ }^{202}$ The concept of imzML is also actively supported by major MS vendors such as Bruker Daltonics, Shimadzu, Waters Corporation and Thermo Scientific which include imzML export options in their respective MSI tools. 
Commercial software tools compatible with imzMLare available and briefly described below, such as Multimaging, MALDIVision, ${ }^{204}$ and SCILS. ${ }^{205}$ A major step towards the acceptance of imzML was the development of open source software. Selected tools are discussed in more detail in the Visualization section below. Each of these tools has specific features. Consequently, imzML provides more flexibility in data processing as the user is no longer limited to proprietary software and can choose the processing option that best fits their purpose.

An important aspect to insure interoperability is the adherence to the data format specifications. In order to facilitate this process, a validator was developed which compares the structure of a given imzML data set with the specifications. ${ }^{206}$ The result is a detailed list of deviations from the standard specifications. The tool can automatically correct for some common problems, such as negative offset values. It also allows users to manually edit and fill in missing metadata in the *.imzML file. The tool also allows users to validate an imzML data set against minimum reporting guidelines. This could be used for in-house requirements or for community-wide guidelines, like the minimum reporting guidelines for MSI, which have been proposed as part of COST Action BM1104. ${ }^{207}$

The data format imzML also enables efficient and easy exchange of MSI data. Full datasets can be shared between collaborators without loss of information which occurs if only sharing images. Results from different instrument platforms can be displayed with identical parameters in one software package for easier comparison. This is demonstrated by a multicenter study on mouse brain sections that included six different instrument platforms. ${ }^{208}$ Instrumentation included different ionization sources such as MALDI and SIMS as well as different mass analyzers such as ToFs, FT-ICRs and Orbitraps from four different vendors. Comparing this data using the respective proprietary software for each instrument is difficult as color scale and interpolation differ significantly. In this study, all data sets were converted to imzML and then displayed in the open source software MSiReader. ${ }^{209}$ As a result, all datasets can be displayed with identical settings for color scale, interpolation and normalization (Figure 5). It also allows all collaboration partners to view and analyze the full data, i.e. mass spectra, facilitating a more detailed discussion of results.

Another possibility for sharing data is the implementation of $i m z M L$ for a public data repository, resulting in the first 'open data' example for MSI. ${ }^{210}$ In other fields, such as genomics and proteomics, raw data is often required as part of a publication, but this is not yet the case for MSI. Reviewers and interested colleagues could reproduce the results of published studies. These 'open' datasets can also be used as example files to develop and test new analysis algorithms. A major development with respect to data sharing is Metaspace. ${ }^{211}$ imzML is an important step towards more efficient collaboration as well as more transparent reporting of results in MSI. In several examples imzML files have been used to combine data from multimodal approaches. ${ }^{212}$ This could also be expanded to vibrational spectroscopy data as shown by the recent data format Raman2imzML. ${ }^{213}$

Visualization. In MSI, several processing steps are needed to obtain a heat map image reflecting the spatial distribution of various molecules throughout a tissue with their relative abundance. Dedicated software is required to handle visualization of this type of dataset. Researchers with different software needs, using different proprietary means of formatting raw data have introduced a lack of uniformity in the field. Numerous MSI visualization and data analysis software are open-source and/or free or commercially available. See Weiskirchen et al. review for a description and evaluation of the various packages dedicated to $\mathrm{MSI}^{214}$ Recently, some efforts have been made to design more user-friendly software requiring less programming knowledge yet programming skills in a chosen code language could be necessary for deeper statistical analysis or an automated data analysis pipeline. As for the images themselves, intensity is often presented with a rainbow color scale which can be misleading as the dynamic range of the scale is too large. ${ }^{215}$ Therefore some software contains other color scheme options, such as viridis. This is important as data analysis and interpretation can be the limiting factor in the throughput of MSI experiments. Some of them are briefly described below. 
One free and open-source software for MSI visualization is MSiReader. ${ }^{216}$ It is built on the MATLAB programming language and incorporates different features for visualization and data analysis, such as a histology overlay tool, normalization options, an annotation tool, an ROI selection and exportation tool for quantification, among others. More recently SpectralAnalysis, ${ }^{217}$ another MATLAB open-source software has been released, which includes multiple collections of algorithms for preprocessing and subsequent multivariate analysis of spectral imaging data. Several data formats are supported such as the well-established imzML, IONTOF export (ToF-SIMS), as well as the ASCIl export from Raman spectroscopy. SpectralAnalysis is capable of handling multiple spectral imaging modalities making it a potential platform to develop and integrate multimodal imaging data. Cardinal, written with the R programming language, is an open-source software for visualization and statistical analysis of MSI experiments. ${ }^{218}$ It allows for multivariate statistical modeling for image segmentation, inference, and model-based visualization as well as providing a foundation for developers to design and implement new methods of computational and statistical analysis. Another opensource R-based software, MassPix, ${ }^{219}$ allows visualization, performs multivariate statistics, and provides putative lipid annotations. METASPACE2020 is a free web-based application for visualization and identification. High mass resolution datasets are required to allow the software to interrogate metabolite databases (HMBD, Lipid Map, etc.). It also incorporates interesting features like a colocalization tool to identify compounds that share same distribution ${ }^{220}$ or an off-sample tool to define sample shape and exclude off sample signals. ${ }^{221}$ It is also a bank of MSI experiment as imzML datasets have to be uploaded, publicly or privately, into METASPACE servers. Datasets (*.imzML and *.ibd files) can then be shared with the METASPACE community. For quantification, msIQuant ${ }^{222}$ is a free software able to handle very large data sets (>50 GB), with different quantitation tools such as automated standard curve generation. Finally, different commercial software like High Definition Imaging (Waters), Multimaging (Imabiotech), IMAGEREVEAL MS (Shimadzu), MALDIVision (PREMIER Biosoft), SCiLS (Bruker), and SurfaceLab (IONTOF) have been developed. They provide user-friendly interfaces with complete data analysis pipelines such as visualization, image registration tools, spatial segmentation, quantification, and/or statistical analysis.

Automated computational and statistical analysis. Validation of statistical differences between samples, or intra-sample, is beneficial in many applications. Choice of the proper univariate analysis will depend on the MSI dataset and the hypothesis of the experiment. A t-test demonstrates quantitative differences for a specific $\mathrm{m} / \mathrm{z}$ between two different samples, ${ }^{223}$ or regions within a tissue section, where an analysis of variance (ANOVA) test can be used to compare three or more regions. ${ }^{148}$ Both of these tests require data to have a Gaussian distribution, but in biological samples, such distributions are not systematically obtained and a nonparametric test like the Mann-Whitney U-Test should then be performed. ${ }^{224}$ Significant differences between samples of a given $\mathrm{m} / \mathrm{z}$ do not imply that this it can be used as biomarker to distinguish different conditions. Specific diagnostic tests such as ROC curve analysis should be applied to determine the statistical difference. ${ }^{225}$ Guo et al. employed such strategies to reveal potential biomarkers of thyroids tumors. ${ }^{224}$ In many cases, a single $\mathrm{m} / \mathrm{z}$ is not enough to properly identify different conditions. A multivariate analysis is required to generate signal fingerprints by combining multiple $\mathrm{m} / \mathrm{z}$ (or biomarkers) and evaluate them in a single ROC curve. ${ }^{226}$

Because of its established history and availability, the most widely applied multivariate analysis for MSI is PCA. ${ }^{227}$ PCA has proven useful for segmenting image data and finding linear correlations between mass peaks. ${ }^{228}$ It works well for exploratory data analysis because it does not assume an underlying causal model, and usually forms an excellent starting point prior to applying other methods to analyze MSI data. ${ }^{229}$ Other multivariate analysis methods that are related to PCA and have been applied to MSI data include PLS, ${ }^{230}$ PCA with partitioning clustering, hierarchical clustering and genetic algorithm analysis, ${ }^{231}$ non-negative matrix factorization (NMF), ${ }^{232} \mathrm{MCR},{ }^{233}$ and maximum autocorrelation factors. ${ }^{234}$ Other multivariate techniques that are often used in conjunction with these dimension reduction techniques include k-means ${ }^{235}$ and hierarchical 
cluster analysis. ${ }^{236}$ ToF-SIMS mass spectra are also notoriously complex and library approaches have so far failed mainly due to its complex ion formation mechanisms. ${ }^{237}$ Furthermore, biological systems are a very complex mixture of molecules that contain the same chemical blocks. For these reasons, multivariate analysis (MVA) tools have been adopted since the early 2000 s to reduce the complexity of the data sets. Graham et al. provide a summary of ToF-SIMS studies that have been carried out using at least one multivariate analysis method. ${ }^{229}$

Over time, researchers have developed various unsupervised data analysis methods; see Verbeeck et al. ${ }^{193}$ for a detailed review on the latest advances. However, these linear multivariate analysis methods that are aimed at data reduction and blind signal separation do not tend to work well for classification problems. ${ }^{238}$ For this reason, people have started exploring supervised ML algorithms such as random forest, ${ }^{115}$ and unsupervised ${ }^{193}$ ML algorithms to analyze MSI data. ToF-SIMS data has been successfully analyzed using various ANNs ${ }^{239}$ in the form of self-organizing maps (SOMs), ${ }^{240}$ or ANNs in combination with t-distributed stochastic neighbor embedding (t-SNE). ${ }^{241}$ The use of ML algorithms makes it possible to reveal chemical differences in MSI data with much greater ease and less human bias but MSI in general is behind the curve when it comes to advanced data analysis compared to other fields. Just like the ToF-SIMS field has been able to learn from the electron microscopy community in terms of sample preparation, it will be necessary for MSI to learn from computer scientists and engineers who have already been applying these techniques for decades.

Biomolecule identification/validation. Once statistical tools have nominated potential $\mathrm{m} / \mathrm{z}$ features of interest, attention turns to towards their identification and validation. Depending on the analyte, there is a multitude of technologies available to transform an $\mathrm{m} / \mathrm{z}$ from a molecular feature to a molecule with biological significance.

Metabolite identification has many challenges to overcome. To compare with proteomics, fragments are not predictable nor are they indicative of the whole metabolite. They are also recurrent among species and therefore unattributable to their biological origin. With these challenges, and a lack of a genetic template, researchers in the metabolomics field began to classify metabolite identification based on experimental rigor. In a recent iteration of this system, Schrimpe-Rutledge et al. describe metabolite identification in five levels of confidence. ${ }^{242}$ This progression of increasing confidence begins with an $m / z$ feature of any mass accuracy in level 5, followed by identifications with a molecular formula in level 4, levels 2 and 3 implementing orthogonal information such MS/MS or retention time while referencing a database and finally, a level 1 identification by confirming structure with a reference standard. In a first step, a widely used approach for metabolite annotation is to apply $\mathrm{m} / \mathrm{z}$ matching, or matching peaks of interest against a molecular database. Various MS databases can be used for annotation such as lipidsMaps, ${ }^{243}$ SwissLipids, ${ }^{244}$ or Human Metabolome Database. ${ }^{245}$ METLIN ${ }^{246}$ more of a technology platform than a regular MS and MS/MS database as it also includes tools for stable-isotopic data and for fragment similarity searches. Other programs such as mummichog ${ }^{247}$ provide a network analysis to predict metabolic relatedness.

However, it has been shown that many $m / z$ features with sub-ppm error mass accuracies had multiple possible identifications. ${ }^{211}$ As crucial as high mass accuracy may be, especially concerning MSI, it alone cannot be used for the validation of an $\mathrm{m} / \mathrm{z}$ feature. Recently, METASPACE developers introduced an FDR-controlled metabolite annotation tool using a metabolite-signal match score. ${ }^{211}$ This tool uses theoretically predicted properties of a metabolite, such as its fine isotopic pattern, and matches it against both spectral and spatial information from MSI data, allowing for a higher confidence identification. To reach a better level of metabolite identification, it is necessary to perform more dedicated approaches like targeted MS/MS imaging or untargeted data-dependent MS/MS imaging. ${ }^{56} \mathrm{~A}$ more common approach is to perform $\mathrm{MS}^{1} \mathrm{MSI}$ and an orthogonal data-dependent LC-MS/MS experiment (typically on a serial section) to annotate unknown molecular features of an image.

Protein identification has several advantages over metabolite identification, namely, there is a genetic code that can be referenced. Proteins also have predictable gas-phase fragmentation patterns, ${ }^{248}$ and enzymatic 
mechanisms, ${ }^{249}$ allowing for searches from in silico databases such as Protalizer, ${ }^{250} \mathrm{MASCOT},{ }^{251}$ and UniProt. ${ }^{252}$ Proteins also benefit from a calculable false discovery rate. In addition, there are many techniques established for the offline validation of identifications. These techniques range in complexity from simple manual extractions, to technologies such as robotic LESA, ${ }^{253}$ hydrogel extraction, ${ }^{254}$ and LCM. ${ }^{255}$ These techniques are not trivial and come with their own challenges and limitations. Ryan et al. cover these protein identification strategies in a recent review. ${ }^{256}$

This illustrates the importance of orthogonal techniques with MSI to validate possible identifications and highlights the potential for techniques such as IM-MS to bring additional information to an MSI experiment, necessary for addressing biological hypotheses.

\section{Multimodal Imaging}

With an understanding of the chemical imaging techniques described above, there is moreover an understanding of their limitations. The physical properties behind these techniques and the information they provide can supplement others in a multimodal workflow.

There are three themes concerning ex vivo multimodal, or correlative or integrated, imaging that appears in the literature. Experiments can be thought of as physically multimodal, multimodal by experimental design and/or computationally multimodal. The physically multimodal involves an analysis of two or more techniques that occurs over the same tissue space; whether it is the same tissue coordinates because one mode has little effect on the subsequent mode, or with offset pixels/sub-sampling. The multimodal by experimental design utilizes the features from one mode and complements it with another, obtaining a dataset more informative beyond any single technology. Lastly, generating computationally combined images from two modes of analysis is another form of multimodal imaging. This registration can range in complexity, from a simple manual overlay of two images with different data to image sharpening, otherwise known as image fusion. With so many methods available to obtain a multimodal dataset, this review will focus on instances of researchers performing a combination of vibrational spectroscopy and MSI and a combination of MSI and MSI, particularly MALDI and ToF-SIMS. Through these examples, recurring themes of image registration and data integration is approached and the utility of multimodal imaging will emerge.

\section{Sample Preparation and Experimental Design for Multimodal Imaging}

There are several reasons to pursue different multimodal strategies. Figure 6 portrays a loose workflow as to how to approach a multimodal experiment. Some instances can be practical in nature, such as for considerations in throughput and costs. To address this, a multimodal experiment can be conducted where one modality is used to guide the other. Such guided acquisition strategies, commonly seen in microscopy with histology-directed workflows, ${ }^{148,257}$ could be applied with chemical imaging techniques, like those described earlier in this review. In most cases, the more implementable technique would be employed to guide a more cumbersome technique. This can be seen when doing a low-resolution image to determine an $\mathrm{ROI}$ requiring higher spatial resolution or more fidelity. It could also be a more advance guidance, like Rabe et al. performing a segmentation of brain tissue based on FTIR image to guide a MALDI-FT-ICR-MSI analysis, reducing acquisition time and load by nearly $98 \%$ (Figure 7,1 ). ${ }^{258}$

Three critical factors concerning the sample preparation of multimodal imaging is the use of a serial section, the level of destructiveness of the complementary modalities and the order of operations in which the analyses occur. Incorporating data from a serial section can be beneficial in a multitude of ways, such as by guiding analysis, providing increased figures of merit such as mass, or spatial resolution, or by facilitating registration, among others. The destructiveness one modality has on the other is another important reason to use a serial section, or close neighbor. Yet, there are levels of destructiveness that are acceptable for same section analysis given the application. For instance, some of the intricacies of MALDI-MSI described earlier, such as washing or matrix/enzyme application, could be thought of as detrimental to another chemical analysis unless the analyte (in terms of sensitivity or delocalization) or morphology is unaffected by the involved sample preparation. This is just one example of acceptable destructiveness. On the flipside, there are issues that perhaps warrant more study in to their affects, such as tissue morphing caused by the ultrahigh 
vacuum of ToF-SIMS imaging. Finally, the order in which these analyses occur should be carefully considered. For instance, in the case of combining FTIR and MALDI-MSI, it is generally thought that FTIR has little effect on the tissue section and would therefore be the first analysis. Yet, Neumann et al. interrogated several different sample preparations and orders of analyses, determining that in their case, analyzing lipids with MALDI-MSI followed by FTIR resulted in the most robust chemical information. ${ }^{259}$ Despite the involved sample preparation of MALDI-MSI, the effectiveness of the unintuitive order of techniques is reflected in their results. As MSI approaches microscopic resolutions, the slightest delocalization will have to be at the forefront of experimental design. This will affect the decision whether to analyze a serial section or the same tissue section, to be covered in the context of specific technologies. As is the case with each of the analytical techniques described thus far, considerations in sample preparation and experimental design should begin with the biological question.

Note that two levels of alignment should be considered related to the biological question. The first one, is the traditional image alignment allowing a simple overlay between images provided by two modalities, as in information between the modalities can be mined using spatial cues or signal cues, or a combination of both. The second level implies raw data alignment called below data integration and allowing access to advanced data processing such as quantitative signal correlation, image sharpening, multimodal classification, and unsupervised multimodal analysis.

Writing this in the present, this section is purposely vague knowing that a new multimodality of techniques thought to be incompatible is likely on the horizon. In keeping, there are fundamental aspects of multimodal imaging, namely image registration and data integration, that are applicable to nearly all ex vivo techniques.

\section{Multimodal data integration for analysis}

The combination of imaging modalities into integrated multimodal datasets opens possibilities along many lines of research. Several analysis factors are implicated in successful multimodal integration. First, image registration (spatial mapping of pixels between modalities) from two or more imaging datasets, is fundamental. Second, once registered, data integration can be uni- or bi-directional, as in information between the modalities can be mined using spatial cues or signal cues, or a combination of both. Finally, visualization of the multimodal data using image overlays or multimodal data analysis plots is critical in the feedback loop ensuring quality registration and subsequent data integration and as well as in interpreting the multimodal imaging results for biological relevance.

Image registration for multimodal workflows. The registration of pixels in one image to those of another links the modalities for integration. Registration of multimodal data can be challenging (Figure 8) because some modalities have very different characteristics (Figure 8.A), can be multiscale (Figure 8.B) (i.e., one dataset sampled at $1 \mu \mathrm{m} /$ pixel and another at $100 \mu \mathrm{m} /$ pixel), and lastly, quantitative evaluation of registration accuracy is not always straightforward. Image characteristics are influential on registration because in automated or manual approaches, one typically selects one representative image from each modality. In spectral imaging, the spectral bands (in MS, the $\mathrm{m} / \mathrm{z}$ bins of the data, in vibrational modalities the $\mathrm{cm}^{-1}$ bins) must be compressed to a single representative image and the decision is not trivial when the detected material and signal can be disparate. In imaging modalities with highly spatially specific signal, this can be even more challenging as it may be that only a small portion of the image contains signal useful for image registration and the corresponding modality may not have signal in these same areas. This dilemma is demonstrated in Figure 8.A where the MSI of rat kidney shows narrow, spatially specific images in kidney sub-regions but the H\&E shows broad staining across the kidney and a combination of stain color and morphology is used to delineate regions. Multi-scale data presents another challenge with the "footprint" of a pixel in one modality sometimes being many hundreds of pixels in another. For instance, many MSI approaches are much less resolved than other complimentary techniques. Figure 8.B shows a theoretical example where an H\&E is sampled at $1 \mu \mathrm{m} / \mathrm{pixel}$ and how what lies beneath a single pixel varies from 10-200 $\mu \mathrm{m} / \mathrm{pixel}$. At $10 \mu \mathrm{m} / \mathrm{pixel}$, only 1 or 2 cells are visible, but at $200 \mu \mathrm{m} / \mathrm{pixel}$, there are possibly hundreds of cells, potentially confounding registration with very different information. These considerations, along with quality assessment, have an effect on how registration is to be performed. Quality assessment is most often qualitative and done by 
subjective evaluation of overlays. For larger structures this is sufficient as many multimodal data will have unmistakable correlation in gross morphology, but suffers at finer resolutions. ${ }^{260,261}$

The most basic practical aspect in registration is the transformation model that computes how pixels are moved from one image to another. Linear models such as rigid (rotation, translation), similarity (scale, rotation, translation), ${ }^{262}$ and affine (scale, shear, rotation, translation) ${ }^{263}$ are appropriate for modalities that can be captured on a single tissue or very close neighboring section such as a histological stain combined with MSI. Advanced non-linear alignments are preferable for very high accuracy registration between images from serial sections where morphology does not change uniformly across the tissue. Most vendor and open source software for MSI allow linear image registration, ${ }^{216}$ mostly built to align MSI with microscopy but if other modalities can be successfully formatted they can also be implemented.

Another registration consideration is the automation of alignment. Image registration in most software is constructed by placing corresponding fiducial "landmarks" based on features of the two images that appear similar to an observer. Software then fits a transformation that minimizes the spatial error between the two sets of landmark points, constrained by the model type (rigid, similarity, affine, non-linear methods). Automated approaches are common in medical imaging, ${ }^{264}$ and have crept into the ex vivo chemical imaging space. Yet, full deployment in chemical imaging has been slower, possibly due the variety of existent software, adapting them from clinical medical image formats to chemical image formats, and the robustness ex vivo data. For instance, most medical images are stored as image matrices, whereas MSI formats are often stored as spectra with an attached matrix of $x$, y positions to form an image map, as described earlier. Vibrational spectroscopy has a similar problem in that the data is not necessary formatted in common image formats. In automatic image registration, image intensity signals or automatically defined descriptors (image keypoints) are used to find commonalities between imaging techniques and are optimized using advanced algorithms that match the descriptors between the images using a transformation model. ${ }^{265}$ Many researchers have incorporated automated registration into their chemical images and have shown impressive multimodal integration as a result, $258,266,267$ but there remains limited direct adoption in open source image analysis software for chemical imaging.

Visualization of modalities. Visualization of multimodality data is important to validate multimodal observations and hypotheses. The paramount task for multimodal image data that provides the most easily actionable information is the image overlay. Overlaying modalities is possible when images are in the same plane. One can then toggle on/off between multiple images, or change the opacity of the individual images to see both modalities simultaneously. Despite the conceptual simplicity of image overlay, the principal challenges faced for most end-users without bioinformatic expertise in this visualization task are software related. Most software is tailored towards a single data type and may allow some additional imaging data to be incorporated, but usually it must be structured in a standard image format (.tiff, .jpeg, .png, etc.). Some recent work, however, has been advanced that provides a single platform for analysis of multiple spectral datasets. ${ }^{217}$ In multimodal integrations using mixed chemical modalities, this usually means that overlay images must be statically generated within a scientific programming environment (such as Matlab, Python or $\mathrm{R}$ ) or static image files must be merged in scientific imaging software like Fiji or commercial non-scientific software like Adobe Photoshop. These convoluted processes lengthen the feedback loop between analysis and visualization of multimodal data but numerous data formatting issues must be answered across many modalities and such a decision is beyond the scope of this review.

Multimodal data integration. After registration of data modalities, the integration can proceed. The simplest workflow is one in which only qualitative spatial cues from one modality drive analysis of another. This integration is applied when expert annotation on one modality provides information on a second (Figure 7.2) or one simply observes trends between the two images qualitatively. In the common example of microscopyMSI integration, one could look at an H\&E stained microscopy image and use histological knowledge to characterize an ROI as "normal" and "diseased" which in turn inform MSI data mining and discovery of chemical signals associated with these regions. ${ }^{268-270}$ Such approaches can also be bi-directional where, in this 
example, microscopy areas that are underneath a grouping of interesting MSI pixels can be used to determine what histological structures are associated with a given signal. ${ }^{271,272}$ Oftentimes these approaches rely on domain expertise such as histopathology to mark or recognize relevant cellular areas on stained microscope images.

In a signal-only approach, direct signal correlations from overlaying pixels of the two modalities are sought. In combinations of vibrational spectroscopy and MSI, this would be looking at which vibrational bands mathematically correlate to MSI peaks. This approach has frequently been useful to find correlations between multiple MSI experiments. ${ }^{273}$ Statistical modeling such as linear correlations can be fit between two sets of registered multimodal pixels to find quantitative associations between a signal in one modality to a signal in another. Multi-scale data is particularly challenging for this integration as a pixel in one modality may overlay many in another, necessitating reducing or expanding one of the sets of pixels before fitting. Lastly, approaches that integrate both spatial information and signal information into a condensed analysis are becoming more common. This more advanced scenario will essentially mine spatial information from the modality and combine it with direct signal correlation (Figure 9). Some image fusion approaches, ${ }^{135,259,274,275}$ and approaches using cell morphology and intensity information, ${ }^{276,277}$ are examples of this approach. Image fusion itself is an important and growing topic (Figure 10.1).

\section{Combining vibrational spectroscopic and mass spectrometry imaging.}

Registration aspects. Vibrational imaging modalities complement the highly specific molecular profiling of mass spectrometric imaging with less specific, but informative chemical images at potentially very high lateral resolution. Qualitative multimodal data examination, where one foregoes image registration, has been successful in a number of applications and is a very simple means to analyze multimodal imaging data. Researchers simply make observations about common features in the multimodal data based on the images. For instance, Raman microscopy has been used to differentiate quinoline isomers in biofilms in previously imaged MALDI-MSI without the need to perform image registration. ${ }^{278}$ Similar qualitative multimodal studies have been successful in combining Raman and ToF-SIMS to study Drosophilia melanogaster development and combining ToF-SIMS, synchrotron-FTIR, synchrotron-UV imaging to study human liver cirrhosis with all three modalities from a single section. ${ }^{279}$ In these limited cases, researchers simply infer correlation from images without calculating relevant metrics.

In more advanced and automated approaches linking vibrational spectroscopy and MSI, image registration is almost always required to prepare modalities. In this domain, fiducial landmark registration using linear transformations (rigid or affine) has been the process of choice to link the modalities. As previously outlined, this approach is most appropriate when modalities are acquired from the same section as is typical in combined vibrational spectroscopy - MSI. Bocklitz et. al. reported a control point registration scheme in multiple publications linking Raman imaging and MSI. ${ }^{280,281}$ They used a mixture of MSI ion and PCA score images as templates to find structural matches between modalities and used the pixel-wise mean Raman image for a template. In all cases the Raman images were at a higher spatial resolution than the MSI images and the transformation and subsequent interpolation of the Raman pixels to MSI coordinate space was used to create a new lower spatial resolution Raman image for analysis. A similar registration approach has been applied to study 3D cell cultures with Raman and MALDI MSI. ${ }^{282}$ QCL-IR and MALDI-MSI have been combined for predictive fusion using a combination of fiducial landmark manual image registration (using PCA derived images for both modalities) and automated image registration processes on the manually initialized transform. ${ }^{259}$ Manual initial registration addresses a common issue in automated registration where computational optimizations become stuck in local minima that are poorly aligned. By starting automated alignment with roughly matched images, improvement to the manual alignment from computational registration is more likely to succeed. Further, the approach described by Neumann et. al. deals with the multiscale problem by upsampling the MSI data to the micron level resolution of QCL-IR which enables predictive fusion for higher spatial resolution. Desorption Electrospray lonization (DESI) MSI and Raman imaging from the same tissue section were combined using a fiducial landmark procedure generating an affine transformation. ${ }^{283}$ Balbekoba et. al. showed the use of microscopy as an intermediate modality for image registration where both FTIR and Laser Ablation Inductively Coupled Plasma (LA-ICP) MS images were 
registered using an affine transformation by fiducial landmarks to a common microscopy image, then the spectral modalities were combined. Such an approach is useful if the target spectral image modalities have highly spatially specific but disparate content where finding corresponding landmarks is difficult. Another very powerful approach is the addition of material to act as fiducials that is visible in the signal of both modalities. Lanni et. al. use this approach to register $\mathrm{C}_{60}$-ToF-SIMS and CRM, adding a chemical microdroplet array visible to both modalities to serve as alignment fiducials. ${ }^{284}$

In total, vibrational spectroscopy in combination with MSI techniques has had limited advancement to automated alignment approaches. However, landmark based image registration has been successful in a broad range of studies with different analytical samples including cell cultures, biofilms, and tissues. With the number of studies emerging as proof of concepts combining these two techniques, and the ever increasing acquisition speed of instrumentation, a stress will eventually be placed on faster multimodal data integration where automated alignment could play a role.

Vibrational spectroscopy and MSI data visualization and integration. Virtually all articles cited in the registration section use static images generated through workflows created with common programming languages, hinting at a possible lack of software that handles interactive image blending after registration.

Data analysis approaches vary and as discussed, a fraction of the studies employed no data mining at all, only qualitative comparisons. However, other studies have deployed a variety of data mining workflows to analyze their multimodal data. In a study of mouse brain combining MALDI-MSI and Raman microscopy, intensity threshold from MSI ion images were used to mask brain regions and mean Raman spectra were extracted so vertical Raman shifts between regions could then be computed. ${ }^{280}$ In this case, the approach did not manually define spatial regions through image annotations from either data before computation but rather used the MSI data itself to define regions. Expert annotation driven data analysis and classification were performed in a pilot study incorporating Raman microscopy and MALDI-MSI from larynx carcinoma. ${ }^{280}$ Here spatial annotations on a registered H\&E microscopy image were used to find MSI and Raman signals associated with histopathologically relevant regions. In turn, Raman and MSI were evaluated for their predictive power of the annotated regions and each served to cross-check the other modality, ultimately demonstrating one modality to determine tissue type (Raman) and the other to investigate changes within each tissue type (MALDI-MSI). Several studies combing vibrational spectroscopy and MSI have used an approach of spectral correlation after registration without expert annotation (Figure 9.1). ${ }^{282,283,285,286}$

Fusion of vibrational spectroscopy and MSI has been achieved with different approaches. Fusion has been performed via PCA for combined Raman and MSI data with great care taken to weight the different spectral data to account for the different dynamic ranges of each data, highlighting an important consideration when combining multimodal data from entirely different sensors (Figure 9.2). ${ }^{285}$ One study using IR and MALDI-MSI utilized high resolution IR to sharpen the MSI image to a higher spatial resolution, using a mid-level fusion approach where Laplacian image pyramids are used to transfer high frequency information from the IR data to a predicted MSI image (Figure 10.2). ${ }^{259}$ They further fused the data by combining principal components from the two modalities, further performing another PCA of the combined, and applied this final analysis to find associated IR and MSI signals. Fused LA-ICP-MS and FTIR data was used for supervised ML in cerebral ischemia using PLS - discriminant analysis and random decision forest algorithms trained from labels on microscopy images. ${ }^{55}$ The authors demonstrate increased discriminative power to distinguish brain regions when combining data.

Apparent from this review of the literature in combining MSI and vibrational spectroscopy is that most data integration solutions require some programming experience to develop and implement. This can be a barrier to many labs as there is complexity in all tasks: data formatting, image registration, modality specific preprocessing, and finally combined data integration and analysis. As such, there is still need for user-friendly software that can ingest multiple data types then combine and analyze them. As mentioned previously, tool was recently developed to address the formatting issue using imzML as a container for Raman microscopy imaging data. ${ }^{213}$ 


\section{Combinations of MSI and MSI.}

Registration aspects. Much like combinations of vibrational spectroscopy and MSI, combinations of MSI modalities are spectral. The principle registration challenges described above persist, namely the choice of a representative image for registration and potential for multi-scale data. Some approaches, however, do not require registration. These approaches generally collect multiple modalities using offsets to pixel coordinates or polarity switching. Thomas et. al. demonstrated 1,5-DAN MALDI matrix as performant for collecting both positive and negative ionization MALDI-MSI data. ${ }^{287} \mathrm{~A}$ checkerboard pattern of coordinates is collected with each modality shifted by a set $\mu \mathrm{m}$ offset in each dimension. This approach was used in conjunction with PLS regression and combined modality data showed utility for analyzing tissue pathology. ${ }^{268}$ Similar data has been collected with on-line polarity switching, eliminating the need for a checkerboard pattern. ${ }^{288,289}$ Others have acquired data multiple times on the same points, varying laser power or polarity, resulting in data that is inherently registered. ${ }^{290-292}$ Again, one group printed different matrices in an array across tissues to collect multiple acquisitions that were inherently registered. ${ }^{293}$ Another group qualitatively evaluated registration by marking up common ROI from two datasets achieved by the sequential digestion of tissue. ${ }^{143}$ An approach to minimize issues from registered data such as the effects of image interpolation on underlying spectral data, is to forego interpolated imaging data in analysis as recently shown by Race et. al. ${ }^{275}$

As described earlier, with MALDI-MSI the choice of matrix partially determines what analytes are ionized and detected. Due to the lack of a "one-size-fits-all" MALDI matrix, combining data from multiple MALDI MSI acquisitions has been demonstrated by many. An early study did multiple MALDI preparations, washes and acquisitions with different matrices to collect multimodal data with the authors using Fiji for manual alignment of static images. ${ }^{294}$ The recently developed RegCombIMS is an R package ${ }^{295}$ extending Cardinal ${ }^{218}$ functionality to register, combine, and analyze multiple MSI datasets for MALDI-MALDI multimodality. The authors demonstrate integration of 4 sequential MALDI-MSI datasets from serial sections collecting lipid data in positive mode, negative mode, using silver-assisted imaging and intact protein imaging. Here, automated registration occurs, incorporating non-linear models through a microscopy intermediate image where each MSI image is computationally registered to a microscopy image on a tissue section between the other modalities. The software offers the user to choose any ion image, PCA score image, or segmentation image from the MSI data to achieve registration. A similar approach employing automated registration of microscopy images, previously registered with MALDI MSI, was used to mine data across modalities while avoiding all interpolation simply by matching pixels to one another and determining their degree of overlap. This lead to the decision of which pixel pairs to use for model fitting. ${ }^{260}$ This method addresses the multi-scale problem by mapping the MALDI-MSI to a higher resolution modality that drives registration and also addresses the need to select a representative image by performing all multimodal registration through monomodal microscopy images.

Combinations of ToF-SIMS and MALDI-MSI is interesting because the contrasting information generated by each while both still providing linkable MS data. Registration of these two data can be challenging because the multi-scale nature. ToF-SIMS is usually sampled at very fine spatial resolutions relative to MALDI-MSI. Microscopy has been shown to be a useful intermediate to achieve these registrations using fiducial landmark approaches between an ion image and the microscope image generating linear transformation models. ${ }^{212}$ In a methodological study, PCA score images of MALDI-MSI and SIMS were used to find a manual registration of the two modalities after the SIMS image (16,000 pixels in $\mathrm{x}$ ) was downsampled to MALDI MSI resolution (48 pixels in $x$ ), reducing the spatial resolution of the SIMS image by a factor of $\sim 300{ }^{296}$ Extrinsic, added gold sputtered fiducials on the tissue surface have been another approach to combine ToF-SIMS and MALDI MSI. ${ }^{297}$ Here, gold sputtered fiducials produce a signal in both modalities that can be used to make fiducial landmarks and build a linear transformation. Borodinov et al. used an etched fiducial in the tissue visible in both MALDI and ToF-SIMS that was automatically identified through a computer vision approach. This etched fiducial was then matched to its counterpart in the ToF-SIMS image automatically and an affine transformation matrix was generated. ${ }^{298}$

Data analysis aspects. Analysis of combined MS and MS data is similar to combinations of vibrational spectroscopy and MSI. Comparisons in this domain are typically aimed at identifying molecular species from 
the modalities that follow a similar pattern, either through uni- or multi-variate analysis. Some studies have been purely qualitative and only examine the observable spatial patterns in images without quantifying the signal relations present. ${ }^{114,212,290,291,299}$ Other studies have looked for pixel-wise mathematical correlation between variables (MS signals). ${ }^{260}$ Overall, studies have focused on spectral-spectral signal analysis regimes in combinations of MS and MS. Combining MALDI-MSI datasets and performing spatial segmentation (spectral clustering, or grouping of spectra into a set number of groups based on their similarity) of the data has been shown and the contribution of multiple datasets examined. ${ }^{295}$ In a fusion application of ToF-SIMS and MALDI$\mathrm{MSI}$, correlated NMF factors were used to find both spectral and spatial factors from multimodal data to predictively sharpen chemical images (Figure 10.1). ${ }^{275}$ Borodinov et al. followed a similar approach linking NMF factors from ToF-SIMS and MALDI-MSI after registration but followed this linking with canonical correlation analysis of the NMF factors to find a set of components that show the multivariate links between data. The authors also described a NMF approach for image sharpening (Figure 10.3). ${ }^{298}$

Overall, most of the studies combining MSI modalities have used qualitative comparisons of the combined data. The more advanced cases use scripting and extensions to existing software to perform advanced data analysis. In either case, these advanced scenarios require some degree of programming experience, highlighting the need for a full open source software solution for multimodal data mining. Visualization was not touched upon for the discussed articles because in every instance, images were statically generated and interacted with. In the case of analyses where data is captured at the exact same positions, dynamic visualization may be possible.

\section{Other Topics in Multimodal Imaging}

Many other chemical imaging techniques have been studied in a multimodal fashion. Microscopy may be the most common as most all ex vivo chemical imaging techniques are related back to a histological stain in the biomedical field. But there are many studies correlating chemical images to immunofluorescence studies and increasing interests in autofluorescence microscopy ${ }^{260}$ with one study even leveraging autofluorescence data to register immunofluorescence and MSI data. ${ }^{300}$ Electron microscopy has also shown to be complementary with ToF-SIMS and has been employed to help evaluate MALDI-MSI crystal size. There have also been studies that correlated ToF-SIMS with electron microscopy, atomic force microscopy and $\mathrm{x}$-ray photoelectric spectroscopy. ${ }^{301,302}$

There being several ionization sources designed for MSI, there are many instances of their correlation with other modalities. For instance, DESI-MSI has paired nicely with MALDI-MSI, yet interestingly limited work has been done combining it with ToF-SIMS or vibrational spectroscopies. Another ionization technique for MSI is LA-ICP, which a review of its integration with other modalities was recently written by Perry et al. ${ }^{303}$ LESA has been another ex vivo technique that has benefited from multimodal imaging, which has aided identification of proteins offline at higher spatial resolutions. ${ }^{304}$

In vivo imaging modalities are another important aspect in biomedical imaging and have been combined in a multimodal fashion amongst themselves. ${ }^{305}$ Correlating them with ex vivo studies is vital. Groups have incorporated in vivo multimodal imaging such as magnetic resonance imaging and positron emission tomography with MSI, such as MALDI and SIMS, ${ }^{306}$ and vibrational spectroscopies.

There is also opportunity to address physical mechanisms behind some of the chemical imaging processes with multimodal imaging. For instance, Van Nuffel et al. interrogated the MALDI process with SIMS imaging. ${ }^{96}$ With a better understanding of the underlying analytical technique employed, there is a better understanding of the biomedical study being conducted with said technique. This is a valuable research investment as it can pinpoint limitations and test many of the assumptions in multimodal imaging, thereby improving sample preparations.

Artificial intelligence (AI), through $\mathrm{ML}$ and deep learning, is taking on an increasingly important role in $\mathrm{MSI}^{193}$ and vibrational spectroscopies, ${ }^{307-309}$ particularly as it pertains to data analysis. Also there is increasing interests in image analysis through $\mathrm{Al}$ which could be impactful in pathology. ${ }^{310}$ And as pathology embraces 
ML in image analysis, multimodal imaging could be more incorporated in to these workflows as the handling of multimodal datasets becomes more ubiquitous.

\title{
Future Challenges
}

The vibrational spectroscopy and MSI communities have seen a great deal of technological advancements that have attracted broader scientific attention from biologists and clinicians. Yet, the combination of these highly complementary analytical techniques, still in its infancy, has value that has yet to be discovered. Just as clinicians and pathologists are now seeing the ability of these techniques alone, soon the potential of multimodal imaging will become more difficult to overlook. But for that to happen, there are some issues that would need to be addressed. There is a need for data analysis software capable of handling and combining such highly dimensional and diverse data. Also, for the workflow to be more widely implemented there is a need for accurate image registration that does not require advanced programming knowledge. Lastly, tools to visualize and interrogate multimodal data will be needed, particularly so other scientific communities can easily access the data generated in order to study their tissue of interest and drive their hypotheses. By addressing these issues, multimodal imaging can have more impact on important biological questions of the day, such as disease progression.

\section{Author information}

\section{Author details.}

* Authors contributed equally.

$\S$ Corresponding Author, E-mail: $\underline{\text { n.desbenoit@cbmn.u-bordeaux.fr }}$

\section{ORCID.}

\author{
Michael Tuck 0000-0002-1888-1323 \\ Landry Blanc : 0000-0001-5622-356X \\ Nathan Heath Patterson : 0000-0002-0064-1583 \\ Sebastiaan Van Nuffel: 0000-0003-1251-3236 \\ Andreas Römpp: 0000-0001-8384-9250 \\ Alain Brunelle: 0000-0001-6526-6481 \\ Sophie Lecomte: 0000-0001-8310-4849 \\ Nicolas Desbenoit: 0000-0002-2242-941X
}

Notes. The authors declare no conflict of interest and no competing financial interest.

\section{Biographies.}

Michael Tuck. Michael Tuck is a PhD candidate for the Centre National de la Recherche at the Universite de Bordeaux (Bordeaux, France). He graduated with a Bachelor's of Science from the University of Tennessee (Knoxville, TN, USA) in 2012 studying biochemistry and molecular biology. He gained mass spectrometry experience in the environmental and forensic fields before joining the Mass Spectrometry Resource Center led by Richard Caprioli at Vanderbilt University (Nashville, TN, USA). As an analyst in the Tissue Imaging Core, he conducted MSI experiments to study complex biological questions. By 2018, he began to apply multimodal imaging approaches to study infectious disease and kidney disease. Since 2020, under the direction of Dr. Nicolas Desbenoit, Michael is focused on multimodal imaging, combining Raman spectroscopy and MALDI MSI, to study tuberculosis.

Landry Blanc, Ph.D. Landry Blanc is a post-doctorate researcher at the Institute of Chemistry \& Biology of Membranes \& Nano-objects (CBMN, UMR 5248, University of Bordeaux). Graduated from Institut Nationale des Sciences Appliquées (INSA) in Toulouse in 2010, he started then a PhD at Institut de Pharmacologie et de 
Biologie Structurale in Toulouse on Tuberculosis (TB) host-pathogen interactions with Jerome Nigou and defended his thesis in 2014. He joined Veronique Dartois group at Rutgers University, NJ, USA to for a postdoctorate on PK/PD studies of anti-TB drugs thanks to Mass Spectrometry and Mass Spectrometry Imaging in 2015. In 2019, he joined CBMN (Bordeaux) thanks to a 2-year fellowship from Fondation pour la Recherche Médicale (FRM). Throughout his career, he acquired a strong expertise in MALDI MSI and its application in biological and medical research.

Rita Touti, Ph.D. Rita Touti is a Research Scientist at L'Oréal. She is developing alternative methodologies to characterize the performance of solar products. After completing MSc degree at ENSCBP engineering graduate school, she obtained her PhD funded by Ceva Santé Animale at the University of Bordeaux. She has worked on the evaluation of the penetration of therapeutic formulations through skin (in vitro, ex vivo and in vivo) using Raman and AP-SMALDI imaging methodologies.

Nathan "Heath" Patterson, Ph.D. Heath Patterson is a Research Instructor in the Department of Biochemistry and part of the Mass Spectrometry Research Center at Vanderbilt University. He received his Ph.D. from the University of Montréal, working under Professor Pierre Chaurand to develop wet lab and computational approaches to analyze complex MALDI MSI data. His main research interests are integration of multimodal data, especially image registration of multiple imaging modalities in 2 and 3 dimensions, and development of open source scientific software to facilitate deployment of computational methods.

Sebastiaan Van Nuffel, Ph.D. Sebastiaan Van Nuffel is an Assistant Research Professor at the Materials Research Institute of the Pennsylvania State University. He obtained his PhD degree at the School of Pharmacy of the University of Nottingham in the UK after completing BSc and MSc degrees in Chemistry at the University of Antwerp in Belgium. After his PhD, he worked as a postdoc for Dr. Alain Brunelle at the French National Center for Scientific Research (CNRS) in 2017 and 2018. Throughout his scientific career, he has built up an extensive expertise in MSI with a special emphasis on time-of-flight secondary ion mass spectrometry and its application in biological and medical research.

Sandrine Villette, Ph.D. Sandrine Villette obtained her PhD degree from the Paris VI Pierre et Marie CurieUniversity in 2004 at the interface between Physics, Chemistry and Biology after completing an engineering degree at the Institut d'Optique Graduate School in Orsay in 2000. She developed a specialization in optics for biology research with a strong emphasis on spectroscopy throughout her scientific career and joined the CNRS in 2007 as a research engineer. She moved in 2017 in the Institute of Chemistry and Biology of Membranes and Nano-objects in Bordeaux to join the "Spectroscopy and imaging of membrane assemblies" team managed by Sophie Lecomte where she is in charge of the spectroscopic facility including FTIR and Raman spectrometers and imagers.

Jean-Christophe Taveau, Ph.D. Jean-Christophe Taveau is a Professor in bio-informatics at the Faculty of Biology, University of Bordeaux, France since 2004. He obtained his PhD degree at the University of Paris VI, Pierre et Marie Curie. After his PhD, he was Assistant Professor in the faculty of Pharmacy in University François Rabelais, Tours. During his career, he mainly worked on single-particle reconstruction and tomography in cryo-electron microscopy in the topics of invertebrate respiratory pigments (Faculty of Pharmacy, University François Rabelais, Tours, France) and of bacterial efflux pumps (Institute of Chemistry \& Biology of Membranes \& Nano-objects (CBMN), CNRS-UMR 5248, University of Bordeaux, France). Because these techniques are strongly based on statistics, he gained expertise in ML methods in the fields of structural bio-informatics and of bio-imaging.

Andreas Römpp, Ph.D. Andreas Römpp is Full Professor and Chair of Bioanalytical Sciences and Food Analysis at University of Bayreuth, Germany. He received his PhD from the University of Mainz for a dissertation at the Max Planck Institute for Chemistry. For the last 20 years, he has worked on method development for high resolution mass spectrometry. In MSI, he has focused on improving the spatial resolution while maintaining - 
or improving - the reliability of compound identification (MSI with high resolution in mass and space). He has always been very interested in collaborative activities such as multicenter studies, reporting guidelines, e.g. in the framework of COST Action BSM1104. For several years, he has pushed and coordinated the common data format imzML. Current projects in MSI are ranging from food analysis to drug development.

Alain Brunelle, Ph.D. Dr. Brunelle obtained his Ph.D. from the Paris-Sud University in Orsay, France, in 1990 under the supervision of Dr. Yvon Le Beyec. He is a Director of Research at the CNRS. Until 2002, his main research interests were centered on the study of the interactions of energetic particles (ions, clusters, molecules, photons) with matter. He particularly studied secondary ion emission and sputtering under the impact of gold clusters having energies ranging from a few keV to several tens of MeV. Between 2002 and 2018 he developed a project of biological MSI by cluster-ToF-SIMS and MALDI-ToF. He moved in 2019 to the Laboratory of Molecular and Structural Archeology (CNRS and Sorbonne University, Paris) with a new project which aims to use ToF-SIMS imaging in the field of cultural heritage, in particular old painting samples.

Sophie Lecomte, Ph.D. She is director of research at CNRS. She received her Ph.D. from the UPMC in Paris (1995). After postdoctoral research at the University of Strathclyde (Glasgow, UK; 1996) and the Max Planck Institut fer Strahlenchemie (Melheim an der Ruhr, Germany; 1996-1998), she joined the Laboratoire de Dynamique, Interactions et Réactivité (LADIR) in Paris as a CNRS researcher (1998). In 2007, she moved to the Institute of Chemistry and Biology of Membranes and Nanoobjects (CBMN) in Bordeaux. She is the leader of the group "Spectroscopy and imaging of membrane assemblies". She has a strong background in vibrational spectroscopy and imaging of biomolecules (ATR-FTIR, PM-IRRAS, Raman, SERS, TERS, NanoIR). Her current research focuses on self-assembling processes of amyloid peptides implicated in Alzheimer diseases.

Nicolas Desbenoit, Ph.D. Dr. N. Desbenoit received his PhD degree in 2009 at Paris Descartes University. After his PhD, N. Desbenoit worked as a postdoc within groups of international renown and leaders in the field of MSI: Dr. A. Brunelle (CNRS-ICSN, France), Prof. Dr. B. Spengler (Justus-Liebig University, Germany), and Prof. Dr. A. Römpp (University of Bayreuth, Germany) and gained a strong expertise in MSI (ToF-SIMS, MALDI-MS) of xenobiotics, drugs and lipids, in the development of multimodal workflow combining vibrational spectroscopies (FTIR, Raman), as well as imzML-based data processing and its applications in biological and biomedical research. Since January 2017, he is "Associate Scientist" (CR-CNRS) at the Institute of Chemistry \& Biology of Membranes \& Nano-objects (CBMN, UMR 5248, University of Bordeaux).

\section{Acknowledgments}

This work was financially supported by the Agence National de la Recherche (France, Grants ANR-19-CE190010601 "MultiRaMaS", ANR-2015-CE29-0007 “DEFIMAGE”), the CNRS (International Emerging Actions (IEA) "MultIRaMaS"). Landry BLANC received funding from the "Fondation pour la Recherche Médicale" (ARF201809007123). 


\section{References}

1. Masyuko, R., Lanni, E. J., Sweedler, J. V. \& Bohn, P. W. Correlated imaging - a grand challenge in chemical analysis. Analyst 138, 1924 (2013).

2. Larkin, P. Infrared and Raman Spectroscopy 2nd Edition. (2018).

3. Gilmore, I. S. SIMS of organics-Advances in 2D and 3D imaging and future outlook. J. Vac. Sci. Technol. A Vacuum, Surfaces, Film. 31, 050819 (2013).

4. Buchberger, A. R., Delaney, K., Johnson, J. \& Li, L. Mass Spectrometry Imaging: A Review of Emerging Advancements and Future Insights. Anal. Chem. 90, 240-265 (2018).

5. Patterson, N. H., Thomas, A. \& Chaurand, P. Monitoring time-dependent degradation of phospholipids in sectioned tissues by MALDI imaging mass spectrometry. J. Mass Spectrom. 49, 622-627 (2014).

6. Giusti, L., Angeloni, C. \& Lucacchini, A. Update on proteomic studies of formalin-fixed paraffin-embedded tissues. Expert Rev. Proteomics 16, 513-520 (2019).

7. Longuespée, R., Casadonte, R., Schwamborn, K. \& Kriegsmann, M. Proteomics in Pathology: The Special Issue. PROTEOMICS Clin. Appl. 13, 1800167 (2019).

8. Gaifulina, R. et al. Label-free Raman spectroscopic imaging to extract morphological and chemical information from a formalin-fixed, paraffin-embedded rat colon tissue section. Int. J. Exp. Pathol. 97, 337-350 (2016).

9. Angel, P. M. et al. Mapping Extracellular Matrix Proteins in Formalin-Fixed, Paraffin-Embedded Tissues by MALDI Imaging Mass Spectrometry. (2018) doi:10.1021/acs.jproteome.7b00713.

10. Scott, D. A. et al. Specific N-linked glycosylation patterns in areas of necrosis in tumor tissues. Int. J. Mass Spectrom. 437, 6976 (2019).

11. Carter, C. L., Jones, J. W., Farese, A. M., MacVittie, T. J. \& Kane, M. A. Inflation-Fixation Method for Lipidomic Mapping of Lung Biopsies by Matrix Assisted Laser Desorption/Ionization-Mass Spectrometry Imaging. Anal. Chem. 88, 4788-4794 (2016).

12. Gaifulina, R. et al. Rapid and complete paraffin removal from human tissue sections delivers enhanced Raman spectroscopic and histopathological analysis. Analyst 145, 1499-1510 (2020).

13. Kallepitis, C. et al. Quantitative volumetric Raman imaging of three dimensional cell cultures. Nat. Commun. 8, 14843 (2017)

14. Dannhorn, A. et al. Universal sample preparation unlocking multimodal molecular tissue imaging. Anal. Chem. 92, acs.analchem.0c00826 (2020).

15. Song, C. L. \& Kazarian, S. G. Three-dimensional depth profiling of prostate tissue by micro ATR-FTIR spectroscopic imaging with variable angles of incidence. Analyst 144, 2954-2964 (2019).

16. Clark, I. \& Torbenson, M. S. Immunohistochemistry and Special Stains in Medical Liver Pathology. Adv. Anat. Pathol. 24, 99109 (2017).

17. Sarri, B. et al. Stimulated Raman histology: one to one comparison with standard hematoxylin and eosin staining. Biomed. Opt. Express 10, 5378 (2019)

18. Andrew Chan, K. L. \& Kazarian, S. G. Attenuated total reflection Fourier-transform infrared (ATR-FTIR) imaging of tissues and live cells. Chem. Soc. Rev. 45, 1850-1864 (2016).

19. Institute, N. A. S. (Athens). Infrared and Raman Spectroscopy of Biological Molecules. (Springer NetherlandsT. Theophanides (Editor), 1979).

20. Pasquini, C. Near infrared spectroscopy: A mature analytical technique with new perspectives - A review. Anal. Chim. Acto 1026, 8-36 (2018).

21. Kuepper, C. et al. Quantum Cascade Laser-Based Infrared Microscopy for Label-Free and Automated Cancer Classification in Tissue Sections. Sci. Rep. 8, 1-10 (2018).

22. Bassan, P. et al. Transmission FT-IR chemical imaging on glass substrates: Applications in infrared spectral histopathology. Anal. Chem. 86, 1648-1653 (2014).

23. Pilling, M. J. et al. Infrared spectral histopathology using haematoxylin and eosin (H\&E) stained glass slides: a major step forward towards clinical translation. Analyst 142, 1258-1268 (2017).

24. Pena, A.-M. M. et al. Imaging and quantifying drug delivery in skin - Part 2: Fluorescence andvibrational spectroscopic imaging methods. Adv. Drug Deliv. Rev. (2020) doi:10.1016/j.addr.2020.03.003.

25. Siebert, F. \& Hildebrandt, P. Vibrational Spectroscopy in Life Science. Vib. Spectrosc. (2007).

26. Chrabaszcz, K. et al. Label-free FTIR spectroscopy detects and visualizes the early stage of pulmonary micrometastasis seeded from breast carcinoma. Biochim. Biophys. Acta - Mol. Basis Dis. 1864, 3574-3584 (2018).

27. Chrabaszcz, K. et al. Tracking extracellular matrix remodeling in lungs induced by breast cancer metastasis. Fourier transform infrared spectroscopic studies. Molecules 25, (2020).

28. Kimber, J. A. \& Kazarian, S. G. Spectroscopic imaging of biomaterials and biological systems with FTIR microscopy or with quantum cascade lasers. Anal. Bioanal. Chem. 409, 5813-5820 (2017).

29. Odularu, A. T. Worthwhile Relevance of Infrared Spectroscopy in Characterization of Samples and Concept of Infrared Spectroscopy-Based Synchrotron Radiation. 2020, (2020).

30. Vongsvivut, J. et al. Synchrotron macro ATR-FTIR microspectroscopy for high-resolution chemical mapping of single cells. Analyst 144, 3226-3238 (2019).

31. Song, C. L. \& Kazarian, S. G. Micro ATR-FTIR spectroscopic imaging of colon biopsies with a large area Ge crystal. Spectrochimica Acta - Part A: Molecular and Biomolecular Spectroscopy vol. 228 (2020).

32. Finlayson, D., Rinaldi, C. \& Baker, M. J. Is Infrared Spectroscopy Ready for the Clinic? Anal. Chem. 91, 12117-12128 (2019). 
33. Dazzi, A. \& Prater, C. B. AFM-IR: Technology and applications in nanoscale infrared spectroscopy and chemical imaging. Chem. Rev. 117, 5146-5173 (2017).

34. Kemel, K. et al. Nanoscale investigation of human skin and study of skin penetration of Janus nanoparticles. International Journal of Pharmaceutics vol. 579 (2020).

35. Marcott, C. et al. Nanoscale infrared (IR) spectroscopy and imaging of structural lipids in human stratum corneum using an atomic force microscope to directly detect absorbed light from a tunable IR laser source. Exp. Dermatol. 22, 419-421 (2013). Pienpinijtham, P., Thammacharoen, C., Naranitad, S. \& Ekgasit, S. Analysis of cosmetic residues on a single human hair by ATR FT-IR microspectroscopy. Spectrochim. Acta - Part A Mol. Biomol. Spectrosc. 197, 230-236 (2018).

37. Bhargava, R., Fernandez, D. C., Hewitt, S. M. \& Levin, I. W. High throughput assessment of cells and tissues: Bayesian classification of spectral metrics from infrared vibrational spectroscopic imaging data. Biochim. Biophys. Acta - Biomembr. 1758, 830-845 (2006).

38. Ali, M. H. et al. A simple model for cell type recognition using 2D-correlation analysis of FTIR images from breast cancer tissue. J. Mol. Struct. 1163, 472-479 (2018).

39. Kole, M. R., Reddy, R. K., Schulmerich, M. V., Gelber, M. K. \& Bhargava, R. Discrete frequency infrared microspectroscopy and imaging with a tunable quantum cascade laser. Anal. Chem. 84, 10366-10372 (2012).

40. Tiwari, S. et al. Towards Translation of Discrete Frequency Infrared Spectroscopic Imaging for Digital Histopathology of Clinical Biopsy Samples. Anal. Chem. 88, 10183-10190 (2016).

41. Wrobel, T. P., Mukherjee, P. \& Bhargava, R. Rapid visualization of macromolecular orientation by discrete frequency midinfrared spectroscopic imaging. Analyst 142, 75-79 (2017).

42. Movasaghi, Z., Rehman, S. \& Rehman, I. U. Raman spectroscopy of biological tissues. Appl. Spectrosc. Rev. 42, 493-541 (2007).

43. Gomes da Costa, S., Richter, A., Schmidt, U., Breuninger, S. \& Hollricher, O. Confocal Raman microscopy in life sciences. Morphologie 103, 11-16 (2019).

44. Touti, R., Noun, M., Guimberteau, F., Lecomte, S. \& Faure, C. What is the fate of multi-lamellar liposomes of controlled size, charge and elasticity in artificial and animal skin? Eur. J. Pharm. Biopharm. 151, 18-31 (2020).

45. Kopeć, M. \& Abramczyk, H. Angiogenesis - a crucial step in breast cancer growth, progression and dissemination by Raman imaging. Spectrochim. Acta - Part A Mol. Biomol. Spectrosc. 198, 338-345 (2018).

46. De Bleye, C. et al. Raman imaging as a new analytical tool for the quality control of the monitoring of osteogenic differentiation in forming 3D bone tissue. J. Pharm. Biomed. Anal. 186, (2020).

47. D'Acunto, M., Gaeta, R., Capanna, R. \& Franchi, A. Contribution of Raman Spectroscopy to Diagnosis and Grading of Chondrogenic Tumors. Sci. Rep. 10, 2155 (2020).

48. Soldevila, F., Dong, J., Tajahuerce, E., Gigan, S. \& de Aguiar, H. B. Fast compressive Raman bio-imaging via matrix completion. Optica 6, 341 (2019).

49. Scotté, C. et al. Assessment of Compressive Raman versus Hyperspectral Raman for Microcalcification Chemical Imaging. Anal. Chem. 90, 7197-7203 (2018).

50. Krafft, C. et al. Label-Free Molecular Imaging of Biological Cells and Tissues by Linear and Nonlinear Raman Spectroscopic Approaches. Angew. Chemie Int. Ed. 56, 4392-4430 (2017).

51. Brinkmann, M. et al. Portable all-fiber dual-output widely tunable light source for coherent Raman imaging. Biomed. Opt. Express 10, 4437 (2019).

52. Gong, L., Zheng, W., Ma, Y. \& Huang, Z. Saturated Stimulated-Raman-Scattering Microscopy for Far-Field Superresolution Vibrational Imaging. Phys. Rev. Appl. 11, 1 (2019).

53. Gong, L., Zheng, W., Ma, Y. \& Huang, Z. Higher-order coherent anti-Stokes Raman scattering microscopy realizes label-free super-resolution vibrational imaging. Nat. Photonics 14, 115-122 (2020).

54. Ingham, J. et al. A novel FTIR analysis method for rapid high-confidence discrimination of esophageal cancer. Infrared Phys. Technol. 102, (2019).

55. Balbekova, A. et al. Fourier Transform Infrared (FT-IR) and Laser Ablation Inductively Coupled Plasma-Mass Spectrometry (LAICP-MS) Imaging of Cerebral Ischemia: Combined Analysis of Rat Brain Thin Cuts Toward Improved Tissue Classification. Appl. Spectrosc. 72, 241-250 (2018).

56. Hansen, R. L. \& Lee, Y. J. Overlapping MALDI-Mass Spectrometry Imaging for In-Parallel MS and MS/MS Data Acquisition without Sacrificing Spatial Resolution. J. Am. Soc. Mass Spectrom. 28, 1910-1918 (2017).

57. Schulze, G. et al. Investigation of Selected Baseline Removal Techniques as Candidates for Automated Implementation. Appl. Spectrosc. 59, 545-574 (2005).

58. Savitzky, A. \& Golay, M. J. E. Smoothing and Differentiation of Data by Simplified Least Squares Procedures. Anal. Chem. 36, 1627-1639 (1964).

59. Gautam, R., Vanga, S., Ariese, F. \& Umapathy, S. Review of multidimensional data processing approaches for Raman and infrared spectroscopy. EPJ Tech. Instrum. 2, 8 (2015).

60. Butler, H. J. et al. Using Raman spectroscopy to characterize biological materials. Nat. Protoc. 11, 664-687 (2016).

61. Das Gupta, S. et al. Raman microspectroscopic analysis of the tissue-specific composition of the human osteochondral junction in osteoarthritis: A pilot study. Acta Biomater. 106, 145-155 (2020).

62. Shi, L. et al. Mid-infrared metabolic imaging with vibrational probes. Nat. Methods 17, 844-851 (2020).

63. Clède, S. \& Policar, C. Metal-carbonyl units for vibrational and luminescence imaging: Towards multimodality. Chem. - A Eur. J. 21, 942-958 (2015).

64. Kästner, B. et al. Infrared Nanospectroscopy of Phospholipid and Surfactin Monolayer Domains. ACS Omega 3, 4141-4147 (2018). 
65. Zhang, L. et al. Spectral tracing of deuterium for imaging glucose metabolism. Nat. Biomed. Eng. 3, 402-413 (2019).

66. Hu, F. et al. Supermultiplexed optical imaging and barcoding with engineered polyynes. Nat. Methods 15, 194-200 (2018).

67. Trevisan, J., Angelov, P. P., Carmichael, P. L., Scott, A. D. \& Martin, F. L. Extracting biological information with computational analysis of Fourier-transform infrared (FTIR) biospectroscopy datasets: current practices to future perspectives. Analyst 137, 3202-3215 (2012).

68. Teh, S. K. et al. Diagnostic potential of near-infrared Raman spectroscopy in the stomach: differentiating dysplasia from normal tissue. Br. J. Cancer 98, 457-465 (2008).

69. Sattlecker, M., Bessant, C., Smith, J. \& Stone, N. Investigation of support vector machines and Raman spectroscopy for lymph node diagnostics. Analyst 135, 895-901 (2010).

70. Song, C. L., Vardaki, M. Z., Goldin, R. D. \& Kazarian, S. G. Fourier transform infrared spectroscopic imaging of colon tissues: evaluating the significance of amide I and $\mathrm{C}-\mathrm{H}$ stretching bands in diagnostic applications with machine learning. Anal. Bioanal. Chem. 411, 6969-6981 (2019).

71. Mayerich, D. et al. Stain-less staining for computed histopathology. Technology 3, 27-31 (2015).

72. Berisha, S. et al. Deep learning for FTIR histology: leveraging spatial and spectral features with convolutional neural networks. Analyst 144, 1642-1653 (2019).

73. Gobinet, C. et al. Digital Dewaxing of Raman Spectral Images of Paraffin-Embedded Human Skin Biopsies Based on Ica and Ncls. 1-5 (2008).

74. Miloudi, L. et al. Confocal Raman spectroscopic imaging for in vitro monitoring of active ingredient penetration and distribution in reconstructed human epidermis model. J. Biophotonics 11, 1-12 (2018).

75. Felten, J. et al. Vibrational spectroscopic image analysis of biological material using multivariate curve resolution-alternating least squares (MCR-ALS). Nat. Protoc. 10, 217-240 (2015).

76. Albro, M. B. et al. Raman spectroscopic imaging for quantification of depth-dependent and local heterogeneities in native and engineered cartilage. npj Regen. Med. 3, 1-11 (2018).

77. Brunelle, A., Touboul, D. \& Laprévote, O. Biological tissue imaging with time-of-flight secondary ion mass spectrometry and cluster ion sources. J. Mass Spectrom. 40, 985-999 (2005).

78. Gilmore, I. S., Heiles, S. \& Pieterse, C. L. Metabolic Imaging at the Single-Cell Scale: Recent Advances in Mass Spectrometry Imaging. Annu. Rev. Anal. Chem. 12, 201-224 (2019).

79. Vickerman, J. C. \& Briggs, D. ToF-SIMS: Materials Analysis by Mass Spectrometry. (SurfaceSpectra, 2013).

80. Touboul, D., Kollmer, F., Niehuis, E., Brunelle, A. \& Laprévote, O. Improvement of biological time-of-flight-secondary ion mass spectrometry imaging with a bismuth cluster ion source. J. Am. Soc. Mass Spectrom. 16, 1608-1618 (2005).

81. Benninghoven, A. Surface analysis by Secondary Ion Mass Spectrometry (SIMS). Surf. Sci. 299-300, 246-260 (1994).

82. Van Vaeck, L., Adriaens, A. \& Gijbels, R. Static secondary ion mass spectrometry: (S-SIMS) part 1. Methodology and structural interpretation. Mass Spectrom. Rev. 18, 1-47 (1999).

83. Fletcher, J. S. et al. A new dynamic in mass spectral imaging of single biological cells. Anal. Chem. 80, 9058-9064 (2008).

84. Cooks, R. G. \& Busch, K. L. Matrix effects, internal energies and MS/MS spectra of molecular ions sputtered from surfaces. Int. J. Mass Spectrom. Ion Phys. 53, 111-124 (1983).

85. Massonnet, P. \& Heeren, R. M. A. A concise tutorial review of TOF-SIMS based molecular and cellular imaging. J. Anal. At. Spectrom. 34, 2217-2228 (2019).

86. Steere, R. L. ELECTRON MICROSCOPY OF STRUCTURAL DETAIL IN FROZEN BIOLOGICAL SPECIMENS. J. Biophys. Biochem. Cytol. 3, 45-60 (1957).

87. Linner, J. G., Livesey, S. A., Harrison, D. S. \& Steiner, A. L. A new technique for removal of amorphous phase tissue water without ice crystal damage: a preparative method for ultrastructural analysis and immunoelectron microscopy. J. Histochem. Cytochem. 34, 1123-1135 (1986).

88. Piwowar, A. M. et al. Effects of Cryogenic Sample Analysis on Molecular Depth Profiles with TOF-Secondary lon Mass Spectrometry. Anal. Chem. 82, 8291-8299 (2010).

89. Altelaar, A. F. M. \& Piersma, S. R. Cellular Imaging Using Matrix-Enhanced and Metal-Assisted SIMS BT - Mass Spectrometry Imaging: Principles and Protocols. in (eds. Rubakhin, S. S. \& Sweedler, J. V) 197-208 (Humana Press, 2010). doi:10.1007/9781-60761-746-4_11.

90. Dowlatshahi Pour, M., Malmberg, P. \& Ewing, A. An investigation on the mechanism of sublimed DHB matrix on molecular ion yields in SIMS imaging of brain tissue. Anal. Bioanal. Chem. 408, 3071-3081 (2016).

91. Körsgen, M. et al. Changes in the molecular ion yield and fragmentation of peptides under various primary ions in ToF-SIMS and matrix-enhanced ToF-SIMS. Biointerphases 11, 02A318 (2016).

92. Angerer, T. B., Dowlatshahi Pour, M., Malmberg, P. \& Fletcher, J. S. Improved Molecular Imaging in Rodent Brain with Timeof-Flight-Secondary Ion Mass Spectrometry Using Gas Cluster Ion Beams and Reactive Vapor Exposure. Anal. Chem. 87, 43054313 (2015).

93. Rabbani, S., Barber, A. M., Fletcher, J. S., Lockyer, N. P. \& Vickerman, J. C. TOF-SIMS with Argon Gas Cluster lon Beams: A Comparison with C60+. Anal. Chem. 83, 3793-3800 (2011).

94. Seah, M. P., Spencer, S. J., Havelund, R., Gilmore, I. S. \& Shard, A. G. Depth resolution at organic interfaces sputtered by argon gas cluster ions: the effect of energy, angle and cluster size. Analyst 140, 6508-6516 (2015).

95. Passarelli, M. K. et al. Single-Cell Analysis: Visualizing Pharmaceutical and Metabolite Uptake in Cells with Label-Free 3D Mass Spectrometry Imaging. Anal. Chem. 87, 6696-6702 (2015).

96. Van Nuffel, S. et al. Insights into the MALDI Process after Matrix Deposition by Sublimation Using 3D ToF-SIMS Imaging. Anal. Chem. 90, 1907-1914 (2018). 
97. Starr, N. J. et al. Enhanced vitamin C skin permeation from supramolecular hydrogels, illustrated using in situ ToF-SIMS 3D chemical profiling. Int. J. Pharm. 563, 21-29 (2019).

98. Benettoni, P. et al. Identification of nanoparticles and their localization in algal biofilm by 3D-imaging secondary ion mass spectrometry. J. Anal. At. Spectrom. 34, 1098-1108 (2019).

99. Vestal, M. L., Juhasz, P. \& Martin, S. A. Delayed extraction matrix-assisted laser desorption time-of-flight mass spectrometry. Rapid Commun. Mass Spectrom. 9, 1044-1050 (1995).

100. Vanbellingen, Q. P. et al. Time-of-flight secondary ion mass spectrometry imaging of biological samples with delayed extraction for high mass and high spatial resolutions. Rapid Commun. Mass Spectrom. 29, 1187-1195 (2015).

101. Vanbellingen, Q. P. et al. Mapping Dicorynia guianensis Amsh. wood constituents by submicron resolution cluster-TOF-SIMS imaging. J. Mass Spectrom. 51, 412-423 (2016).

102. Blockhuys, S., Malmberg, P. \& Wittung-Stafshede, P. Copper distribution in breast cancer cells detected by time-of-flight secondary ion mass spectrometry with delayed extraction methodology. Biointerphases 13, $06 \mathrm{E} 412$ (2018).

103. Henss, A. et al. High resolution imaging and 3D analysis of Ag nanoparticles in cells with ToF-SIMS and delayed extraction. Biointerphases 13, 03B410 (2018).

104. Touboul, D., Brunelle, A. \& Laprévote, O. Structural analysis of secondary ions by post-source decay in time-of-flight secondary ion mass spectrometry. Rapid Commun. Mass Spectrom. 20, 703-709 (2006).

105. Fletcher, J. S., Kotze, H. L., Armitage, E. G., Lockyer, N. P. \& Vickerman, J. C. Evaluating the challenges associated with time-offight secondary ion mass spectrometry for metabolomics using pure and mixed metabolites. Metabolomics 9, 535-544 (2013).

106. Fisher, G. L. et al. A New Method and Mass Spectrometer Design for TOF-SIMS Parallel Imaging MS/MS. Anal. Chem. 88, 64336440 (2016).

107. Passarelli, M. K. et al. The 3D OrbiSIMS-label-free metabolic imaging with subcellular lateral resolution and high massresolving power. Nat. Methods 14, 1175-1183 (2017).

108. Bruinen, A. L. et al. Identification and High-Resolution Imaging of $\alpha$-Tocopherol from Human Cells to Whole Animals by TOFSIMS Tandem Mass Spectrometry. J. Am. Soc. Mass Spectrom. 29, 1571-1581 (2018).

109. Fu, T. et al. Tandem Mass Spectrometry Imaging and in Situ Characterization of Bioactive Wood Metabolites in Amazonian Tree Species Sextonia rubra. Anal. Chem. 90, 7535-7543 (2018).

110. Shi, Y. et al. Mass Spectrometric Imaging Reveals Temporal and Spatial Dynamics of Bioactive Lipids in Arteries Undergoing Restenosis. J. Proteome Res. 18, 1669-1678 (2019).

111. Aoyagi, S. et al. OrbiSIMS Imaging Identifies Molecular Constituents of the Perialgal Vacuole Membrane of Paramecium bursaria with Symbiotic Chlorella variabilis. Anal. Chem. 91, 14545-14551 (2019).

112. Zhang, J. et al. Cryo-OrbiSIMS for 3D Molecular Imaging of a Bacterial Biofilm in Its Native State. Anal. Chem. 92, 9008-9015 (2020).

113. Newell, C. L., Vorng, J.-L., MacRae, J. I., Gilmore, I. S. \& Gould, A. P. Cryogenic OrbiSIMS Localizes Semi-Volatile Molecules in Biological Tissues. Angew. Chemie Int. Ed. n/a, (2020).

114. Schaepe, K. et al. Imaging of Lipids in Native Human Bone Sections Using TOF-Secondary lon Mass Spectrometry, Atmospheric Pressure Scanning Microprobe Matrix-Assisted Laser Desorption/Ionization Orbitrap Mass Spectrometry, and OrbitrapSecondary Ion Mass Spectrometry. Anal. Chem. 90, 8856-8864 (2018).

115. Van Nuffel, S. et al. Multimodal Imaging Mass Spectrometry to Identify Markers of Pulmonary Arterial Hypertension in Human Lung Tissue Using MALDI-ToF, ToF-SIMS, and Hybrid SIMS. Anal. Chem. 53, 19 (2020).

116. Muramoto, S., Forbes, T. P., van Asten, A. C. \& Gillen, G. Test Sample for the Spatially Resolved Quantification of Illicit Drugs on Fingerprints Using Imaging Mass Spectrometry. Anal. Chem. 87, 5444-5450 (2015).

117. Bich, C., Touboul, D. \& Brunelle, A. Study of experimental variability in TOF-SIMS mass spectrometry imaging of biological samples. Int. J. Mass Spectrom. 337, 43-49 (2013).

118. Desbenoit, N. et al. Localisation and quantification of benzalkonium chloride in eye tissue by TOF-SIMS imaging and liquid chromatography mass spectrometry. Anal. Bioanal. Chem. 405, 4039-4049 (2013).

119. Philipsen, M. H., Sämfors, S., Malmberg, P. \& Ewing, A. G. Relative quantification of deuterated omega-3 and -6 fatty acids and their lipid turnover in PC12 cell membranes using TOF-SIMS. J. Lipid Res. 59, 2098-2107 (2018).

120. Najafinobar, N. et al. ToF-SIMS mediated analysis of human lung tissue reveals increased iron deposition in COPD (GOLD IV) patients. Sci. Rep. 9, 10060 (2019).

121. Kern, C. et al. Investigation of strontium transport and strontium quantification in cortical rat bone by time-of-flight secondary ion mass spectrometry. J. R. Soc. Interface 16, 20180638 (2019).

122. Smith, S. C., Zhou, C., Stevie, F. A. \& Garcia, R. Imaging and quantitative analysis of insecticide in mosquito net fibers using Time-of-Flight Secondary lon Mass Spectrometry (ToF-SIMS). PLoS One 13, 1-19 (2018).

123. Lin, H.-Y., Dyakov, Y. A., Lee, Y. T. \& Ni, C.-K. Temperature Dependence of Desorbed lons and Neutrals and lonization Mechanism of Matrix-Assisted Laser Desorption/Ionization. J. Am. Soc. Mass Spectrom. (2020) doi:10.1021/jasms.0c00101.

124. Calvano, C. D., Monopoli, A., Cataldi, T. R. I. \& Palmisano, F. MALDI matrices for low molecular weight compounds: an endless story? Anal. Bioanal. Chem. 410, 4015-4038 (2018).

125. Perry, W. J. et al. Uncovering matrix effects on lipid analyses in MALDI imaging mass spectrometry experiments. J. Mass Spectrom. 55, e4491 (2020).

126. Trimpin, S., Räder, H. J. \& Müllen, K. Investigations of theoretical principles for MALDI-MS derived from solvent-free sample preparation. Int. J. Mass Spectrom. 253, 13-21 (2006)

127. Yang, J. \& Caprioli, R. M. Matrix sublimation/recrystallization for imaging proteins by mass spectrometry at high spatial resolution. Anal. Chem. 83, 5728-5734 (2011). 
128. Gemperline, E., Rawson, S. \& Li, L. Optimization and Comparison of Multiple MALDI Matrix Application Methods for Small Molecule Mass Spectrometric Imaging. Anal. Chem. 86, 10030-10035 (2014).

129. Warren, A. D., Mitchell, D. J. \& Gates, P. J. Methodologies for the airbrush application of MALDI matrices. Eur. J. Mass Spectrom. 24, 89-95 (2018).

130. Bouschen, W., Schulz, O., Eikel, D. \& Spengler, B. Matrix vapor deposition/recrystallization and dedicated spray preparation for high-resolution scanning microprobe matrix-assisted laser desorption/ionization imaging mass spectrometry (SMALDI-MS) of tissue and single cells. Rapid Commun. Mass Spectrom. 24, 355-364 (2010).

131. Stoeckli, M. \& Staab, D. Reproducible Matrix Deposition for MALDI MSI Based on Open-Source Software and Hardware. J. Am. Soc. Mass Spectrom. 26, 911-914 (2015).

132. Yang, J., Norris, J. L. \& Caprioli, R. Novel vacuum stable ketone-based matrices for high spatial resolution MALDI imaging mass spectrometry. J. Mass Spectrom. 53, 1005-1012 (2018).

133. Spraggins, J. M. et al. MALDI FTICR IMS of intact proteins: Using mass accuracy to link protein images with proteomics data. J. Am. Soc. Mass Spectrom. 26, 947-985 (2015).

134. Prentice, B. M., Ryan, D. J., Van De Plas, R., Caprioli, R. M. \& Spraggins, J. M. Enhanced Ion Transmission Efficiency up to m / z 24000 for MALDI Protein Imaging Mass Spectrometry. Anal. Chem. 90, 5090-5099 (2018).

135. Van de Plas, R., Yang, J., Spraggins, J. \& Caprioli, R. M. Image fusion of mass spectrometry and microscopy: a multimodality paradigm for molecular tissue mapping. Nat. Methods 12, 366-372 (2015).

136. Longuespée, R. et al. Tissue Proteomics for the Next Decade? Towards a Molecular Dimension in Histology. Omi. A J. Integr. Biol. 18, 539-552 (2014).

137. Groseclose, M. R., Andersson, M., Hardesty, W. M. \& Caprioli, R. M. Identification of proteins directly from tissue: In situ tryptic digestions coupled with imaging mass spectrometry. J. Mass Spectrom. 42, 254-262 (2007).

138. Judd, A. M. et al. A recommended and verified procedure for in situ tryptic digestion of formalin-fixed paraffin-embedded tissues for analysis by matrix-assisted laser desorption/ionization imaging mass spectrometry. J. Mass Spectrom. 54, 716-727 (2019).

139. Huber, K. et al. Approaching cellular resolution and reliable identification in mass spectrometry imaging of tryptic peptides. Anal. Bioanal. Chem. 410, 5825-5837 (2018).

140. Briggs, M. T. et al. MALDI Mass Spectrometry Imaging of Early- and Late-Stage Serous Ovarian Cancer Tissue Reveals StageSpecific N- Glycans. Proteomics 19, 1800482 (2019).

141. Holst, S. et al. Linkage-Specific in Situ Sialic Acid Derivatization for N-Glycan Mass Spectrometry Imaging of Formalin-Fixed Paraffin-Embedded Tissues. Anal. Chem. 88, 5904-5913 (2016).

142. Carter, C. L. et al. MALDI-MSI spatially maps N-glycan alterations to histologically distinct pulmonary pathologies following irradiation. Sci. Rep. 10, 11559 (2020).

143. Heijs, B. et al. Multimodal Mass Spectrometry Imaging of N-Glycans and Proteins from the Same Tissue Section. (2016) doi:10.1021/acs.analchem.6b01739.

144. Arnaud, B. et al. Imaging Study by Mass Spectrometry of the Spatial Variation of Cellulose and Hemicellulose Structures in Corn Stalks. J. Agric. Food Chem. 68, 4042-4050 (2020).

145. Fernández-Vega, A. et al. Optimization of a MALDI-Imaging protocol for studying adipose tissue-associated disorders. Talanto 219, 121184 (2020).

146. Rzagalinski, I. \& Volmer, D. A. Quantification of low molecular weight compounds by MALDI imaging mass spectrometry - A tutorial review. Biochim. Biophys. Acta - Proteins Proteomics 1865, 726-739 (2017).

147. Tobias, F. \& Hummon, A. B. Considerations for MALDI-based Quantitative Mass Spectrometry Imaging Studies The Ohio State University, Columbus, Ohio 43210-1132, United States of America Corresponding author: Fernando Tobias. (2020) doi:10.1021/acs.jproteome.0c00443.

148. Blanc, L. et al. High-resolution mapping of fluoroquinolones in TB rabbit lesions reveals specific distribution in immune cell types. Elife (2018) doi:10.7554/eLife.41115.

149. Deininger, S.-O. et al. Normalization in MALDI-TOF imaging datasets of proteins: practical considerations. Anal. Bioanal. Chem. 401, 167-181 (2011).

150. Fonville, J. M. et al. Robust Data Processing and Normalization Strategy for MALDI Mass Spectrometric Imaging. Anal. Chem. 84, 1310-1319 (2012).

151. Bokhart, M. T. et al. Quantitative mass spectrometry imaging of emtricitabine in cervical tissue model using infrared matrixassisted laser desorption electrospray ionization. Anal. Bioanal. Chem. 407, 2073-2084 (2015).

152. Hamm, G. et al. Quantitative mass spectrometry imaging of propranolol and olanzapine using tissue extinction calculation as normalization factor. J. Proteomics 75, 4952-4961 (2012).

153. Prideaux, B. et al. The association between sterilizing activity and drug distribution into tuberculosis lesions. Nat. Med. 21 1223-1227 (2015).

154. Schulz, S., Becker, M., Groseclose, M. R., Schadt, S. \& Hopf, C. Advanced MALDI mass spectrometry imaging in pharmaceutical research and drug development. Curr. Opin. Biotechnol. 55, 51-59 (2019).

155. Signor, L. et al. Analysis of erlotinib and its metabolites in rat tissue sections by MALDI quadrupole time-of-flight mass spectrometry. J. Mass Spectrom. 42, 900-909 (2007).

156. Chumbley, C. W. et al. Absolute Quantitative MALDI Imaging Mass Spectrometry: A Case of Rifampicin in Liver Tissues. Anal. Chem. 88, 2392-2398 (2016).

157. Jadoul, L., Longuespée, R., Noël, A. \& De Pauw, E. A spiked tissue-based approach for quantification of phosphatidylcholines in brain section by MALDI mass spectrometry imaging. Anal. Bioanal. Chem. 407, 2095-2106 (2015). 
158. Barry, J. A., Groseclose, M. R. \& Castellino, S. Quantification and assessment of detection capability in imaging mass spectrometry using a revised mimetic tissue model. Bioanalysis 11, 1099-1116 (2019).

159. Hansen, H. T. \& Janfelt, C. Aspects of Quantitation in Mass Spectrometry Imaging Investigated on Cryo-Sections of Spiked Tissue Homogenates. Anal. Chem. 88, 11513-11520 (2016).

160. Zimmerman, M., Blanc, L., Chen, P.-Y., Dartois, V. \& Prideaux, B. Spatial Quantification of Drugs in Pulmonary Tuberculosis Lesions by Laser Capture Microdissection Liquid Chromatography Mass Spectrometry (LCM-LC/MS). J. Vis. Exp. (2018) doi:10.3791/57402.

161. Dilillo, M. et al. Mass Spectrometry Imaging, Laser Capture Microdissection, and LC-MS/MS of the Same Tissue Section. J. Proteome Res. 16, 2993-3001 (2017).

162. Heeren, R. M. A. Getting the picture: The coming of age of imaging MS. Int. J. Mass Spectrom. 377, $672-680$ (2015).

163. Spengler, B., Hubert, M. \& Kaufmann, R. MALDI Ion Imaing and Biological Ion Imaging with a new Scanning UV-Laser Microprobe. Proc. 42nd ASMS Conf. Mass Spectrom. Allied Top. p 1041 (1994).

164. Caprioli, R. M., Farmer, T. B. \& Gile, J. Molecular Imaging of Biological Samples: Localization of Peptides and Proteins Using MALDI-TOF MS. Anal. Chem. 69, 4751-4760 (1997).

165. Zavalin, A. et al. Direct imaging of single cells and tissue at sub-cellular spatial resolution using transmission geometry MALDI MS. Journal of Mass Spectrometry vol. 47 1473-1481 (2012).

166. Kompauer, M., Heiles, S. \& Spengler, B. Atmospheric pressure MALDI mass spectrometry imaging of tissues and cells at 1.4 $\mu \mathrm{m}$ lateral resolution. Nat. Methods 14, 90-96 (2016).

167. Heiles, S., Kompauer, M., Müller, M. A. \& Spengler, B. Atmospheric-Pressure MALDI Mass Spectrometry Imaging at $213 \mathrm{~nm}$ Laser Wavelength. J. Am. Soc. Mass Spectrom. 31, 326-335 (2020).

168. Niehaus, M., Soltwisch, J., Belov, M. E. \& Dreisewerd, K. Transmission-mode MALDI-2 mass spectrometry imaging of cells and tissues at subcellular resolution. Nat. Methods 16, 925-931 (2019).

169. Laiko, V. V., Baldwin, M. A. \& Burlingame, A. L. Atmospheric Pressure Matrix-Assisted Laser Desorption/lonization Mass Spectrometry. Anal. Chem. 72, 652-657 (2000).

170. Römpp, A. \& Spengler, B. Mass spectrometry imaging with high resolution in mass and space. Histochem. Cell Biol. 139, 759$783(2013)$.

171. Steven, R. T. et al. Construction and testing of an atmospheric-pressure transmission-mode matrix assisted laser desorption ionisation mass spectrometry imaging ion source with plasma ionisation enhancement. Anal. Chim. Acta 1051, 110-119 (2019).

172. Soltwisch, J. et al. Mass spectrometry imaging with laser-induced postionization. Science (80-. ). 348, $211-215$ (2015).

173. Spraggins, J. M. et al. High-Performance Molecular Imaging with MALDI Trapped Ion-Mobility Time-of-Flight (timsTOF) Mass Spectrometry. Anal. Chem. 91, 14552-14560 (2019).

174. Barré, F. P. Y. Y. et al. Enhanced sensitivity using maldi imaging coupled with laser postionization (maldi-2) for pharmaceutical research. Anal. Chem. 91, 10840-10848 (2019).

175. Spivey, E. C., McMillen, J. C., Ryan, D. J., Spraggins, J. M. \& Caprioli, R. M. Combining MALDI-2 and transmission geometry laser optics to achieve high sensitivity for ultra-high spatial resolution surface analysis. J. Mass Spectrom. 54, 366-370 (2019).

176. Boskamp, M. S. \& Soltwisch, J. Charge Distribution between Different Classes of Glycerophospolipids in MALDI-MS Imaging. Anal. Chem. 92, 5222-5230 (2020).

177. Eiersbrock, F. B., Orthen, J. M. \& Soltwisch, J. Validation of MALDI-MS imaging data of selected membrane lipids in murine brain with and without laser postionization by quantitative nano-HPLC-MS using laser microdissection. Anal. Bioanal. Chem. (2020) doi:10.1007/s00216-020-02818-y.

178. Hulme, H. et al. Simultaneous mass spectrometry imaging of multiple neuropeptides in the brain and alterations induced by experimental parkinsonism and L-DOPA therapy. Neurobiol. Dis. 137, 104738 (2020).

179. Ogrinc Potočnik, N., Fisher, G. L., Prop, A. \& Heeren, R. M. A. A. Sequencing and Identification of Endogenous Neuropeptides with Matrix-Enhanced Secondary Ion Mass Spectrometry Tandem Mass Spectrometry. Anal. Chem. 89, 8223-8227 (2017).

180. Shariatgorji, M. et al. Direct Targeted Quantitative Molecular Imaging of Neurotransmitters in Brain Tissue Sections. Neuron 84, 697-707 (2014).

181. Shariatgorji, M. et al. Comprehensive mapping of neurotransmitter networks by MALDI-MS imaging. Nat. Methods 16, 10211028 (2019).

182. Fülöp, A. et al. New Derivatization Reagent for Detection of free Thiol-groups in Metabolites and Proteins in Matrix-Assisted Laser Desorption/Ionization Mass Spectrometry Imaging. Anal. Chem. 92, 6224-6228 (2020).

183. Manier, M. L. et al. Reagent precoated targets for rapid in-tissue derivatization of the anti-tuberculosis drug isoniazid followed by MALDI imaging mass spectrometry. J. Am. Soc. Mass Spectrom. 22, 1409-1419 (2011).

184. Prentice, B. et al. Dynamic Range Expansion by Gas-Phase Ion Fractionation and Enrichment for Imaging Mass Spectrometry. (2020) doi:10.26434/CHEMRXIV.12318926.V1.

185. Soltwisch, J. et al. MALDI-2 on a Trapped Ion Mobility Quadrupole Time-of-Flight Instrument for Rapid Mass Spectrometry Imaging and Ion Mobility Separation of Complex Lipid Profiles. Anal. Chem. acs.analchem.0c01747 (2020) doi:10.1021/acs.analchem.0c01747.

186. Gabelica, V. et al. Recommendations for reporting ion mobility Mass Spectrometry measurements. Mass Spectrom. Rev. 38, 291-320 (2019).

187. Djambazova, K. et al. Resolving the Complexity of Spatial Lipidomics Using MALDI TIMS Imaging Mass Spectrometry. doi:10.26434/chemrxiv.12331652.v2.

188. Prentice, B. M. \& Caprioli, R. M. The Need for Speed in Matrix-Assisted Laser Desorption/Ionization Imaging Mass 
Spectrometry. Postdoc J. a J. Postdr. Res. Postdr. Aff. 4, 3-13 (2016).

189. Spraggins, J. M. et al. Next-generation technologies for spatial proteomics: Integrating ultra-high speed MALDI-TOF and high mass resolution MALDI FTICR imaging mass spectrometry for protein analysis. Proteomics 16, 1678-1689 (2016).

190. Ogrinc Potočnik, N., Porta, T., Becker, M., Heeren, R. M. A. \& Ellis, S. R. Use of advantageous, volatile matrices enabled by next-generation high-speed matrix-assisted laser desorption/ionization time-of-flight imaging employing a scanning laser beam. Rapid Commun. Mass Spectrom. 29, 2195-2203 (2015).

191. Barré, F. et al. Faster raster matrix-assisted laser desorption/ionization mass spectrometry imaging of lipids at high lateral resolution. Int. J. Mass Spectrom. 437, 38-48 (2019).

192. Alexandrov, T. Spatial Metabolomics and Imaging Mass Spectrometry in the Age of Artificial Intelligence. Annu. Rev. Biomed. Data Sci. 3, 61-87 (2020).

193. Verbeeck, N., Caprioli, R. M. \& Van de Plas, R. Unsupervised machine learning for exploratory data analysis in imaging mass spectrometry. Mass Spectrom. Rev. 39, 245-291 (2020).

194. Norris, J. L. et al. Processing MALDI mass spectra to improve mass spectral direct tissue analysis. Int. J. Mass Spectrom. 260, 212-221 (2007).

195. Olsen, J. V. et al. Parts per Million Mass Accuracy on an Orbitrap Mass Spectrometer via Lock Mass Injection into a C-trap. Mol. Cell. Proteomics 4, 2010-2021 (2005).

196. Brochu, F. et al. Mass spectra alignment using virtual lock-masses. Sci. Rep. 9, 8469 (2019).

197. Taylor, A. J., Dexter, A. \& Bunch, J. Exploring Ion Suppression in Mass Spectrometry Imaging of a Heterogeneous Tissue. Anal. Chem. 90, 5637-5645 (2018).

198. Alexandrov, T. MALDI imaging mass spectrometry: statistical data analysis and current computational challenges. $B M C$ Bioinformatics 13, S11 (2012).

199. Race, A. M., Steven, R. T., Palmer, A. D., Styles, I. B. \& Bunch, J. Memory Efficient Principal Component Analysis for the Dimensionality Reduction of Large Mass Spectrometry Imaging Data Sets. Anal. Chem. 85, 3071-3078 (2013).

200. Martens, L. et al. mzML-a Community Standard for Mass Spectrometry Data. Mol. Cell. Proteomics 10, R110.000133 (2011).

201. Römpp, A. et al. imzML: Imaging Mass Spectrometry Markup Language: A Common Data Format for Mass Spectrometry Imaging. in 205-224 (2011). doi:10.1007/978-1-60761-987-1_12.

202. Schramm, T. et al. imzML - A common data format for the flexible exchange and processing of mass spectrometry imaging data. J. Proteomics 75, 5106-5110 (2012).

203. Race, A. M., Styles, I. B. \& Bunch, J. Inclusive sharing of mass spectrometry imaging data requires a converter for all. J. Proteomics 75, 5111-5112 (2012).

204. PREMIERBiosoft. 'MALDIVision.' Retrieved 3 July 2012, from. http://www.premierbiosoft.com/maldi-tissueimaging/index.html. (2012).

205. Daltonics, B. 'SCiLS product information' Retrieved the 12 October 2020, from. https://scils.de/products/. (2020).

206. Race, A. M. \& Römpp, A. Error-Free Data Visualization and Processing through imzML and mzML Validation. Anal. Chem. 90, 13378-13384 (2018).

207. McDonnell, L. A. et al. Discussion point: reporting guidelines for mass spectrometry imaging. Anal. Bioanal. Chem. 407, 20352045 (2015).

208. Römpp, A. et al. Mass spectrometry imaging of biological tissue: an approach for multicenter studies. Anal. Bioanal. Chem. 407, 2329-2335 (2015).

209. Robichaud, G., Garrard, K. P., Barry, J. A. \& Muddiman, D. C. MSiReader: An Open-Source Interface to View and Analyze High Resolving Power MS Imaging Files on Matlab Platform. J. Am. Soc. Mass Spectrom. 24, 718-721 (2013).

210. Römpp, A. et al. A public repository for mass spectrometry imaging data. Anal. Bioanal. Chem. 407, $2027-2033$ (2015).

211. Palmer, A. et al. FDR-controlled metabolite annotation for high-resolution imaging mass spectrometry. Nat. Methods 14, 5760 (2017).

212. Desbenoit, N., Walch, A., Spengler, B., Brunelle, A. \& Römpp, A. Correlative mass spectrometry imaging, applying time-offlight secondary ion mass spectrometry and atmospheric pressure matrix-assisted laser desorption/ionization to a single tissue section. Rapid Commun. Mass Spectrom. 32, 159-166 (2018).

213. lakab, S. A., Sementé, L., García-Altares, M., Correig, X. \& Ràfols, P. Raman2imzML converts Raman imaging data into the standard mass spectrometry imaging format. BMC Bioinformatics 21, 448 (2020).

214. Weiskirchen, R., Weiskirchen, S., Kim, P. \& Winkler, R. Software solutions for evaluation and visualization of laser ablation inductively coupled plasma mass spectrometry imaging (LA-ICP-MSI) data: a short overview. J. Cheminform. 11, 16 (2019).

215. Race, A. M. \& Bunch, J. Optimisation of colour schemes to accurately display mass spectrometry imaging data based on human colour perception. Anal. Bioanal. Chem. 407, 2047-2054 (2015).

216. Bokhart, M. T., Nazari, M., Garrard, K. P. \& Muddiman, D. C. MSiReader v1.0: Evolving Open-Source Mass Spectrometry Imaging Software for Targeted and Untargeted Analyses. J. Am. Soc. Mass Spectrom. 29, 8-16 (2018).

217. Race, A. M. et al. SpectralAnalysis: Software for the Masses. Anal. Chem. 88, 9451-9458 (2016).

218. Bemis, K. D. et al. Cardinal : an R package for statistical analysis of mass spectrometry-based imaging experiments: Fig. 1. Bioinformatics 31, 2418-2420 (2015).

219. Bond, N. J., Koulman, A., Griffin, J. L. \& Hall, Z. massPix: an R package for annotation and interpretation of mass spectrometry imaging data for lipidomics. Metabolomics 13, 128 (2017).

220. Ovchinnikova, K., Stuart, L., Rakhlin, A., Nikolenko, S. \& Alexandrov, T. ColocML: machine learning quantifies co-localization between mass spectrometry images. Bioinformatics 36, 3215-3224 (2020).

221. Ovchinnikova, K., Kovalev, V., Stuart, L. \& Alexandrov, T. OffsampleAl: artificial intelligence approach to recognize off-sample 
mass spectrometry images. BMC Bioinformatics 21, 129 (2020).

222. Källback, P., Nilsson, A., Shariatgorji, M. \& Andrén, P. E. msIQuant - Quantitation Software for Mass Spectrometry Imaging Enabling Fast Access, Visualization, and Analysis of Large Data Sets. Anal. Chem. 88, 4346-4353 (2016).

223. Yajima, Y. et al. Region of Interest analysis using mass spectrometry imaging of mitochondrial and sarcomeric proteins in acute cardiac infarction tissue. Sci. Rep. 8, 7493 (2018).

224. Guo, S. et al. Tissue imaging and serum lipidomic profiling for screening potential biomarkers of thyroid tumors by matrixassisted laser desorption/ionization-Fourier transform ion cyclotron resonance mass spectrometry. Anal. Bioanal. Chem. 406, 4357-4370 (2014).

225. Hoo, Z. H., Candlish, J. \& Teare, D. What is an ROC curve? Emerg. Med. J. 34, 357-359 (2017).

226. Wang, M.-C. \& Li, S. ROC analysis for multiple markers with tree-based classification. Lifetime Data Anal. 19, $257-277$ (2013).

227. Trindade, G. F., Abel, M. L. \& Watts, J. F. simsMVA: A tool for multivariate analysis of ToF-SIMS datasets. Chemom. Intell. Lab. Syst. 182, 180-187 (2018).

228. Van Nuffel, S., Parmenter, C., Scurr, D. J., Russell, N. A. \& Zelzer, M. Multivariate analysis of 3D ToF-SIMS images: method validation and application to cultured neuronal networks. Analyst 141, 90-95 (2016).

229. Graham, D. J. \& Castner, D. G. Multivariate Analysis of ToF-SIMS Data from Multicomponent Systems: The Why, When, and How. Biointerphases 7, 49 (2012).

230. Hook, A. L., Williams, P. M., Alexander, M. R. \& Scurr, D. J. Multivariate ToF-SIMS image analysis of polymer microarrays and protein adsorption. Biointerphases 10, 19005 (2015)

231. Brulet, M. et al. Lipid mapping of colonic mucosa by cluster TOF-SIMS imaging and multivariate analysis in cftr knockout mice. J. Lipid Res. 51, 3034-3045 (2010).

232. Trindade, G. F., Abel, M.-L., Lowe, C., Tshulu, R. \& Watts, J. F. A Time-of-Flight Secondary lon Mass Spectrometry/Multivariate Analysis (ToF-SIMS/MVA) Approach To Identify Phase Segregation in Blends of Incompatible but Extremely Similar Resins. Anal. Chem. 90, 3936-3941 (2018).

233. Jaumot, J. \& Tauler, R. Potential use of multivariate curve resolution for the analysis of mass spectrometry images. Analyst 140, 837-846 (2015).

234. Henderson, A., Fletcher, J. S. \& Vickerman, J. C. A comparison of PCA and MAF for ToF-SIMS image interpretation. Surf. Interface Anal. 41, 666-674 (2009).

235. Palmer, A. D., Bunch, J. \& Styles, I. B. The Use of Random Projections for the Analysis of Mass Spectrometry Imaging Data. J. Am. Soc. Mass Spectrom. 26, 315-322 (2015).

236. Urbini, M. et al. ToF-SIMS and principal component analysis of lipids and amino acids from inflamed and dysplastic human colonic mucosa. Anal. Bioanal. Chem. 409, 6097-6111 (2017).

237. Gelb, L. D. \& Walker, A. V. Toward understanding weak matrix effects in TOF SIMS. J. Vac. Sci. Technol. B 36, 03F127 (2018).

238. Madiona, R. M. T. et al. Multivariate analysis of ToF-SIMS data using mass segmented peak lists. Surf. Interface Anal. 50, 713728 (2018).

239. Matsuda, K. \& Aoyagi, S. Time-of-flight secondary ion mass spectrometry analysis of hair samples using unsupervised artificial neural network. Biointerphases 15, 21013 (2020).

240. Gardner, W. et al. ToF-SIMS and Machine Learning for Single-Pixel Molecular Discrimination of an Acrylate Polymer Microarray. Anal. Chem. 92, 6587-6597 (2020).

241. Dexter, A. et al. Training a neural network to learn other dimensionality reduction removes data size restrictions in bioinformatics and provides a new route to exploring data representations. bioRxiv 2020.09.03.269555 (2020) doi:10.1101/2020.09.03.269555.

242. Schrimpe-Rutledge, A. C., Codreanu, S. G., Sherrod, S. D. \& McLean, J. A. Untargeted Metabolomics Strategies-Challenges and Emerging Directions. J. Am. Soc. Mass Spectrom. 27, 1897-1905 (2016)

243. Sud, M. et al. LMSD: LIPID MAPS structure database. Nucleic Acids Res. 35, D527-D532 (2007).

244. Aimo, L. et al. The SwissLipids knowledgebase for lipid biology. Bioinformatics 31, 2860-2866 (2015).

245. Wishart, D. S. et al. HMDB 4.0: the human metabolome database for 2018. Nucleic Acids Res. 46, D608-D617 (2018).

246. Guijas, C. et al. METLIN: A Technology Platform for Identifying Knowns and Unknowns. Anal. Chem. 90, 3156-3164 (2018).

247. Caspi, R. et al. The MetaCyc database of metabolic pathways and enzymes and the BioCyc collection of pathway/genome databases. Nucleic Acids Res. 44, D471-D480 (2016).

248. Mekecha, T. T., Amunugama, R. \& McLuckey, S. A. Ion Trap Collision-Induced Dissociation of Human Hemoglobin $\alpha$-Chain Cations. J. Am. Soc. Mass Spectrom. 17, 923-931 (2006).

249. Zhang, Y., Fonslow, B. R., Shan, B., Baek, M. C. \& Yates, J. R. Protein analysis by shotgun/bottom-up proteomics. Chemical Reviews vol. 113 2343-2394 (2013).

250. Heaven, M. R. et al. Micro-Data-Independent Acquisition for High-Throughput Proteomics and Sensitive Peptide Mass Spectrum Identification. Anal. Chem. 90, 8905-8911 (2018).

251. Perkins, D. N., Pappin, D. J. C., Creasy, D. M. \& Cottrell, J. S. Probability-based protein identification by searching sequence databases using mass spectrometry data. in Electrophoresis vol. 20 3551-3567 (Wiley-VCH Verlag, 1999).

252. UniProt: a worldwide hub of protein knowledge. Nucleic Acids Res. 47, D506-D515 (2019).

253. Wisztorski, M. et al. Spatially-resolved protein surface microsampling from tissue sections using liquid extraction surface analysis. Proteomics 16, 1622-1632 (2016).

254. Ahmed, E. M. Hydrogel: Preparation, characterization, and applications: A review. Journal of Advanced Research vol. 6 105$121(2015)$

255. Datta, S. et al. Laser capture microdissection: Big data from small samples. Histology and Histopathology vol. $301255-1269$ 
(2015).

256. Ryan, D. J., Spraggins, J. M. \& Caprioli, R. M. Protein identification strategies in MALDI imaging mass spectrometry: a brief review. Current Opinion in Chemical Biology vol. 48 64-72 (2019).

257. Patterson, N. H. et al. Next Generation Histology-Directed Imaging Mass Spectrometry Driven by Autofluorescence Microscopy. Anal. Chem. 90, 12404-12413 (2018).

258. Rabe, J.-H. H. et al. Fourier Transform Infrared Microscopy Enables Guidance of Automated Mass Spectrometry Imaging to Predefined Tissue Morphologies. Sci. Rep. 8, 1-11 (2018).

259. Neumann, E. K. et al. Multimodal Chemical Analysis of the Brain by High Mass Resolution Mass Spectrometry and Infrared Spectroscopic Imaging. Anal. Chem. 90, 11572-11580 (2018).

260. Patterson, N. H., Tuck, M., Van De Plas, R. \& Caprioli, R. M. Advanced Registration and Analysis of MALDI Imaging Mass Spectrometry Measurements through Autofluorescence Microscopy. Anal. Chem. 90, 12395-12403 (2018).

261. Rappez, L. et al. Spatial single-cell profiling of intracellular metabolomes in situ: Supplementary Data S1. bioRxiv 510222 (2019) doi:10.1101/510222.

262. Blanc, L., Lenaerts, A., Dartois, V. \& Prideaux, B. Visualization of Mycobacterial Biomarkers and Tuberculosis Drugs in Infected Tissue by MALDI-MS Imaging. Anal. Chem. (2018) doi:10.1021/acs.analchem.8b00985.

263. Lotz, J. M. J. J. M. et al. Integration of 3D multimodal imaging data of a head and neck cancer and advanced feature recognition. Biochim. Biophys. Acta - Proteins Proteomics 1865, 946-956 (2017).

264. Viergever, M. A. et al. A survey of medical image registration - under review. Medical Image Analysis vol. 33 140-144 (2016).

265. Song, G., Han, J., Zhao, Y., Wang, Z. \& Du, H. A Review on Medical Image Registration as an Optimization Problem. Curr. Med. Imaging Rev. 13, (2017).

266. Abdelmoula, W. M. et al. Automatic generic registration of mass spectrometry imaging data to histology using nonlinear stochastic embedding. Anal. Chem. 86, 9204-9211 (2014).

267. Kong, K. et al. Diagnosis of tumors during tissue-conserving surgery with integrated autofluorescence and Raman scattering microscopy. Proc. Natl. Acad. Sci. U. S. A. 110, 15189-15194 (2013).

268. Thomas, A. et al. Histology-driven data mining of lipid signatures from multiple imaging mass spectrometry analyses: Application to human colorectal cancer liver metastasis biopsies. Anal. Chem. 85, 2860-2866 (2013).

269. Deutskens, F., Yang, J. \& Caprioli, R. M. High spatial resolution imaging mass spectrometry and classical histology on a single tissue section. J. Mass Spectrom. 46, 568-571 (2011).

270. Chaurand, P. et al. Integrating Histology and Imaging Mass Spectrometry. Anal. Chem. 76, 1145-1155 (2004).

271. Bemis, K. D. et al. Probabilistic segmentation of mass spectrometry (MS) images helps select important ions and characterize confidence in the resulting segments. Mol. Cell. Proteomics 15, 1761-1772 (2016).

272. Guo, D. et al. Deep multiple instance learning classifies subtissue locations in mass spectrometry images from tissue-level annotations. Bioinformatics 36, i300-i308 (2020).

273. Holzlechner, M., Bonta, M., Lohninger, H., Limbeck, A. \& Marchetti-Deschmann, M. Multisensor Imaging-From Sample Preparation to Integrated Multimodal Interpretation of LA-ICPMS and MALDI MS Imaging Data. Anal. Chem. 90, 8831-8837 (2018).

274. Guo, A., Chen, Z., Li, F., Li, W. \& Luo, Q. Towards More Reliable Unsupervised Tissue Segmentation Via Integrating Mass Spectrometry Imaging and Hematoxylin-Erosin Stained Histopathological Image. bioRxiv 2020.07.17.208025 (2020) doi:10.1101/2020.07.17.208025.

275. Race, A. M. et al. Correlative Hyperspectral Imaging Using a Dimensionality-Reduction-Based Image Fusion Method. Anal. Chem. 92, 10979-10988 (2020).

276. Picard de Muller, G., Ait-Belkacem, R., Bonnel, D., Longuespée, R. \& Stauber, J. Automated Morphological and Morphometric Analysis of Mass Spectrometry Imaging Data: Application to Biomarker Discovery. J. Am. Soc. Mass Spectrom. 28, 2635-2645 (2017).

277. Castellanos-García, L. J., Gokhan Elci, S. \& Vachet, R. W. Reconstruction, analysis, and segmentation of LA-ICP-MS imaging data using Python for the identification of sub-organ regions in tissues. Analyst 145, 3705-3712 (2020).

278. Baig, N. F. et al. Multimodal chemical imaging of molecular messengers in emerging Pseudomonas aeruginosa bacterial communities. Analyst 140, 6544-6552 (2015).

279. Petit, V. W. et al. Multimodal Spectroscopy Combining Time-of-Flight-Secondary lon Mass Spectrometry, Synchrotron-FT-IR, and Synchrotron-UV Microspectroscopies on the Same Tissue Section. Anal. Chem. 82, 3963-3968 (2010).

280. Bocklitz, T. W. et al. Deeper Understanding of Biological Tissue: Quantitative Correlation of MALDI-TOF and Raman Imaging. Anal. Chem. 85, 10829-10834 (2013).

281. Bocklitz, T. et al. Novel workflow for combining Raman spectroscopy and MALDI-MSI for tissue based studies. Anal. Bioanal. Chem. 407, 7865-7873 (2015).

282. Ahlf, D. R., Masyuko, R. N., Hummon, A. B. \& Bohn, P. W. Correlated mass spectrometry imaging and confocal Raman microscopy for studies of three-dimensional cell culture sections. Analyst 139, 4578-4585 (2014).

283. Bergholt, M. S. et al. Correlated Heterospectral Lipidomics for Biomolecular Profiling of Remyelination in Multiple Sclerosis. ACS Cent. Sci. 4, 39-51 (2018).

284. Lanni, E. J. et al. Correlated Imaging with C 60 -SIMS and Confocal Raman Microscopy: Visualization of Cell-Scale Molecular Distributions in Bacterial Biofilms. Anal. Chem. 86, 10885-10891 (2014).

285. Ryabchykov, O., Popp, J. \& Bocklitz, T. Fusion of MALDI Spectrometric Imaging and Raman Spectroscopic Data for the Analysis of Biological Samples. Front. Chem. 6, (2018).

286. Lasch, P. \& Noda, I. Two-Dimensional Correlation Spectroscopy for Multimodal Analysis of FT-IR, Raman, and MALDI-TOF MS 
Hyperspectral Images with Hamster Brain Tissue. Anal. Chem. 89, 5008-5016 (2017).

287. Thomas, A., Charbonneau, J. L., Fournaise, E. \& Chaurand, P. Sublimation of new matrix candidates for high spatial resolution imaging mass spectrometry of lipids: Enhanced information in both positive and negative polarities after 1,5diaminonapthalene deposition. Anal. Chem. 84, 2048-2054 (2012).

288. Korte, A. R. \& Lee, Y. J. Multiplex mass spectrometric imaging with polarity switching for concurrent acquisition of positive and negative ion images. J. Am. Soc. Mass Spectrom. 24, 949-955 (2013).

289. Nazari, M. \& Muddiman, D. C. Polarity switching mass spectrometry imaging of healthy and cancerous hen ovarian tissue sections by infrared matrix-assisted laser desorption electrospray ionization (IR-MALDESI). Analyst 141, 595-605 (2016).

290. Kaya, I. et al. Novel Trimodal MALDI Imaging Mass Spectrometry (IMS3) at $10 \mu \mathrm{m}$ Reveals Spatial Lipid and Peptide Correlates Implicated in Aß Plaque Pathology in Alzheimer's Disease. ACS Chem. Neurosci. 8, 2778-2790 (2017).

291. Kaya, I., Samfors, S., Levin, M., Boren, J. \& Fletcher, J. S. Multimodal MALDI Imaging Mass Spectrometry Reveals Spatially Correlated Lipid and Protein Changes in Mouse Heart with Acute Myocardial Infarction. J. Am. Soc. Mass Spectrom. (2020) doi:10.1021/jasms.0c00245.

292. Fincher, J. A., Korte, A. R., Yadavilli, S., Morris, N. J. \& Vertes, A. Multimodal imaging of biological tissues using combined MALDI and NAPA-LDI mass spectrometry for enhanced molecular coverage. Analyst (2020) doi:10.1039/d0an00836b.

293. Urbanek, A., Hölzer, S., Knop, K., Schubert, U. S. \& von Eggeling, F. Multigrid MALDI mass spectrometry imaging (mMALDI MSI). Anal. Bioanal. Chem. 408, 3769-3781 (2016).

294. Steven, R. T. \& Bunch, J. Repeat MALDI MS imaging of a single tissue section using multiple matrices and tissue washes. Anal. Bioanal. Chem. 405, 4719-4728 (2013).

295. Patterson, N. H., Yang, E., Kranjec, E.-A. A. \& Chaurand, P. Co-registration and analysis of multiple imaging mass spectrometry datasets targeting different analytes. Bioinformatics 35, 1261-1262 (2019).

296. Eijkel, G. B. et al. Correlating MALDI and SIMS imaging mass spectrometric datasets of biological tissue surfaces. Surf. Interface Anal. 41, 675-685 (2009).

297. Ogrinc Potočnik, N., Škrášková, K., Flinders, B., Pelicon, P. \& Heeren, R. M. A. Gold sputtered fiducial markers for combined secondary ion mass spectrometry and MALDI imaging of tissue samples. Anal. Chem. 86, 6781-6785 (2014).

298. Borodinov, N., Lorenz, M., King, S. T., levlev, A. V. \& Ovchinnikova, O. S. Toward nanoscale molecular mass spectrometry imaging via physically constrained machine learning on co-registered multimodal data. npj Comput. Mater. 6, 1-8 (2020).

299. Fu, T. et al. Biosynthetic investigation of $\psi$-lactones in Sextonia rubra wood using in situ TOF-SIMS MS/MS imaging to localize and characterize biosynthetic intermediates. Sci. Rep. 9, 1928 (2019).

300. Jones, M. A. et al. Discovering new lipidomic features using cell type specific fluorophore expression to provide spatial and biological specificity in a multimodal workflow with MALDI Imaging Mass Spectrometry. Anal. Chem. (2020) doi:10.1021/acs.analchem.0c00446.

301. Jiang, H., Kilburn, M. R., Decelle, J. \& Musat, N. NanoSIMS chemical imaging combined with correlative microscopy for biological sample analysis. Curr. Opin. Biotechnol. 41, 130-135 (2016).

302. Liu, W. et al. Correlative surface imaging reveals chemical signatures for bacterial hotspots on plant roots. Analyst 145, 393$401(2020)$

303. Perry, W. J. et al. Integrated molecular imaging technologies for investigation of metals in biological systems: A brief review. Curr. Opin. Chem. Biol. 55, 127-135 (2020).

304. Ryan, D. J. et al. MicroLESA: Integrating Autofluorescence Microscopy, In Situ Micro-Digestions, and Liquid Extraction Surface Analysis for High Spatial Resolution Targeted Proteomic Studies. (2019) doi:10.1021/acs.analchem.8b05889.

305. Wu, M. \& Shu, J. Multimodal Molecular Imaging: Current Status and Future Directions. Contrast Media and Molecular Imaging vol. 2018 (2018).

306. Amstalden Van Hove, E. R. et al. Multi-modal mass spectrometric imaging of small molecules reveals distinct spatio-molecular signatures in differentially metastatic breast tumor models HHS Public Access. doi:10.1158/0008-5472.CAN-10-0360.

307. Kananenka, A. A., Yao, K., Corcelli, S. A. \& Skinner, J. L. Machine Learning for Vibrational Spectroscopic Maps. J. Chem. Theory Comput. 15, 6850-6858 (2019).

308. Rodner, E. et al. Fully convolutional networks in multimodal nonlinear microscopy images for automated detection of head and neck carcinoma: A pilot study. Head Neck hed.25489 (2018) doi:10.1002/hed.25489.

309. Pradhan, P. et al. Semantic Segmentation of Non-linear Multimodal Images for Disease Grading of Inflammatory Bowel Disease: A SegNet-based Application. in Proceedings of the 8th International Conference on Pattern Recognition Applications and Methods 396-405 (SCITEPRESS - Science and Technology Publications, 2019). doi:10.5220/0007314003960405.

310. Madabhushi, A. \& Lee, G. Image analysis and machine learning in digital pathology: Challenges and opportunities. Medical Image Analysis vol. 33 170-175 (2016). 


\section{Figures and legends}

Figure 1: Vibrational and Mass Spectrometry Multimodal Imaging Publication Trend. The graph shows the numbers of PubMed-indexed publications in a given year. Blue dashed line: ("Mass spectrometry"); Blue line: ("Mass spectrometry imaging" or "Imaging mass spectrometry"); Red dashed line: ("vibrational spectroscopy" or Raman or "Fourier transform infrared" Red line: ("vibrational spectroscopy" or RAMAN or "Fourier transform infrared" and Imaging; Purple line: ("mass spectrometry" or "vibrational spectroscopy" or Raman or "Fourier transform infrared" and "multimodal imaging").

\section{Popularity of different technologies}

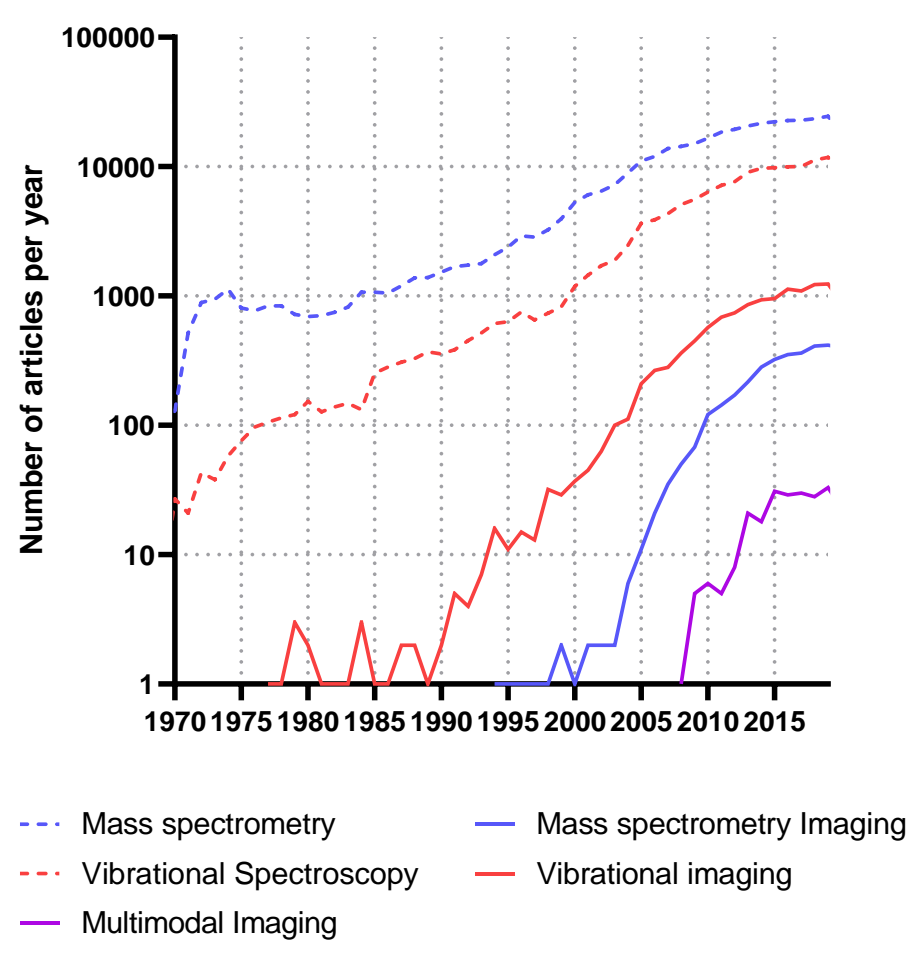


Figure 2: Schematic Confocal Raman workflow, from the sample preparation to data processing. The sample is deposited without any preparation on the glass slide. The analyzed area (yellow rectangle) are chosen on the optical image (red square). Single spectrum can be recorded (yellow cross) or set of spectra can be recorded (on $x$ and $y$ lines) and imaging will be generated using different data processing. For the univariate analysis: left image is based obtained by the integration of the amide I band (indicative of the protein) $1700-1600 \mathrm{~cm}^{-1}$, right image is based on the intensity of a deuterated chain of lipids $\left(2000-2300 \mathrm{~cm}^{-1}\right)$. The K-means analysis allows to determine the areas with the similar Raman spectra (similar compositions), each color correspond to a specific Raman spectrum. In this example, it reveals the different composition of the stratum corneum, epidermis and dermis of the skin.

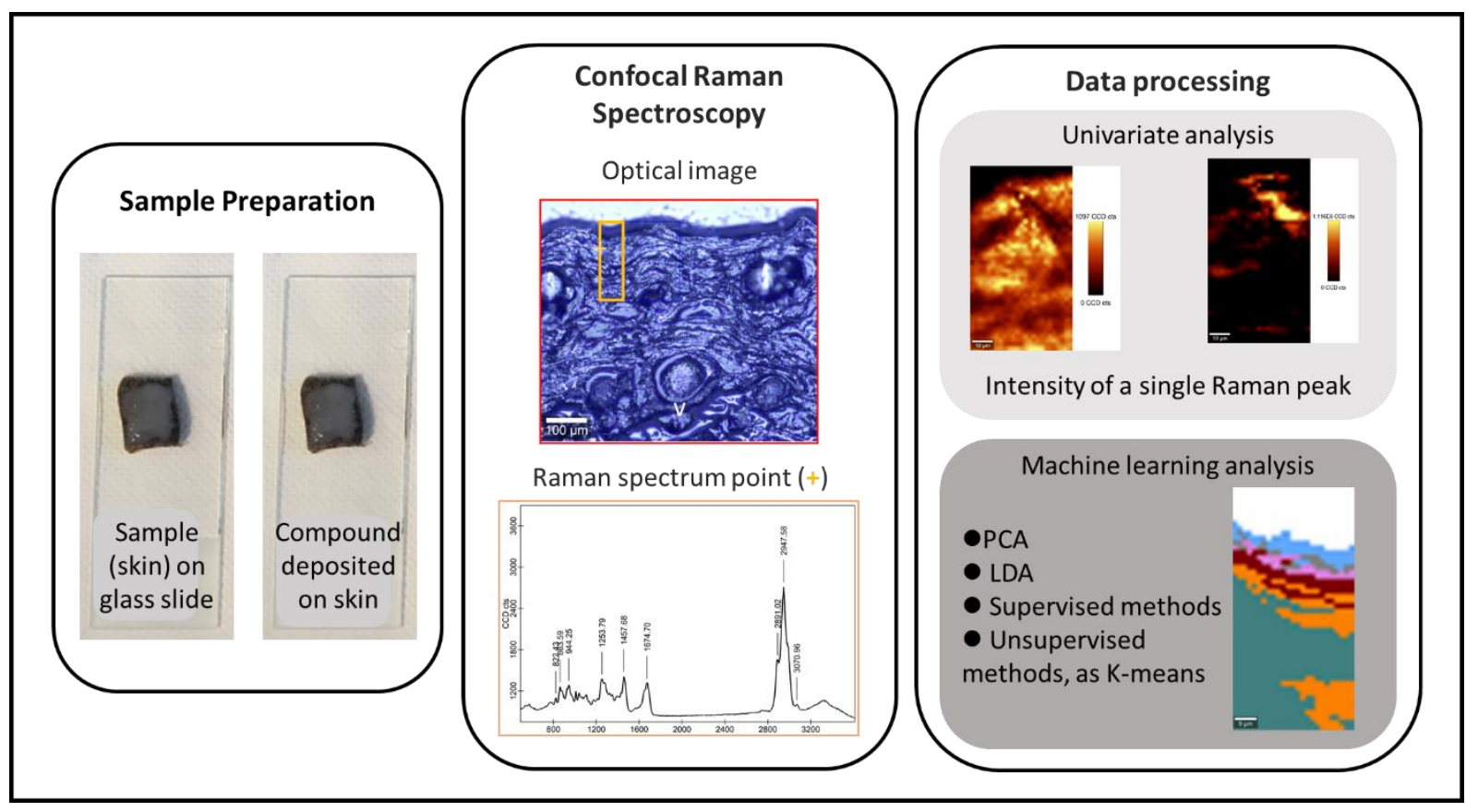


Figure 3: Ion images and MS/MS spectrum recorded at the surface of a wood cross section. (a) A combined MS $\left(M S^{1}\right)$ and $M S / M S\left(M S^{2}\right)$ false color overlay of aromatic fragment ions $\left(M S^{1}, m / z 77.04\right.$ and 91.06 , red), the total ions of $\mathrm{m} / \mathrm{z} 421\left[\mathrm{M}^{\mathrm{P3}}+\mathrm{K}\right]^{+}\left(\mathrm{MS}^{2}\right.$, green), and lignin fragment ions $\left(\mathrm{MS}^{1}, \mathrm{~m} / \mathrm{z} 137.06,151.08,167.07\right.$ and 181.05 , blue). The field-of-view is $300 \mu \mathrm{m} \times 300 \mu \mathrm{m}$ acquired at $512 \times 512$ image pixels. The blue square indicates the $70 \mu \mathrm{m} \times 70 \mu \mathrm{m}$ area expanded in the gray-scale images of panels (b) through (i). (b) MS $\mathrm{S}^{1}$ image of ${ }^{41} \mathrm{~K}^{+}$ions. (c) $M S^{1}$ image of the aromatic ion sum. (d) $M S^{1}$ image of the lignin ion sum. (e) $M S^{1}$ image of the rubrynolide metabolite ions, compressed to $128 \times 128$ pixels. (f) $\mathrm{MS}^{1}$ image of the suspected biosynthetic $\left[\mathrm{M}^{\mathrm{P3}}+\mathrm{H}\right]^{+}$ions, compressed to $128 \times 128$ pixels. $(\mathrm{g})$ Tandem $\mathrm{MS}\left(\mathrm{MS}^{2}\right)$ image of $\mathrm{m} / \mathrm{z} 421\left[\mathrm{M}^{\mathrm{P3}}+\mathrm{K}\right]^{+}$ions (i.e. the sum of all product ions and unfragmented precursor ions). (h) $\mathrm{MS}^{2}$ image of the product ions (i.e. $\mathrm{m} / \mathrm{z} 303,225$, 125 and 43) arising from the $\left[\mathrm{M}^{P 3}+K\right]^{+}$ions, compressed to $128 \times 128$ pixels. (i) $\mathrm{MS}^{2}$ image of the $\mathrm{K}^{+}$product ions $\left(\mathrm{m} / \mathrm{z}\right.$ 39) arising from the $\left[\mathrm{M}^{P 3}+K\right]^{+}$ions. (j) $M S^{2}$ spectrum arising from $\mathrm{CID}$ fragmentation of $\left[\mathrm{M}^{p 3}+K\right]^{+}(\mathrm{m} / \mathrm{z}$ 421) ions. The proposed $\gamma$-lactone molecular structure for $M^{P 3}$ is shown in the inset. Reproduced from $F u, T$;; Touboul, D.; Della-Negra, S.; Houël, E.; Amusant, N.; Duplais, C.; Fisher, G.L.; Brunelle, A. Anal. Chem. 2018, 90, 7535-7543 (ref 109). Copyright 2018 American Chemical Society.
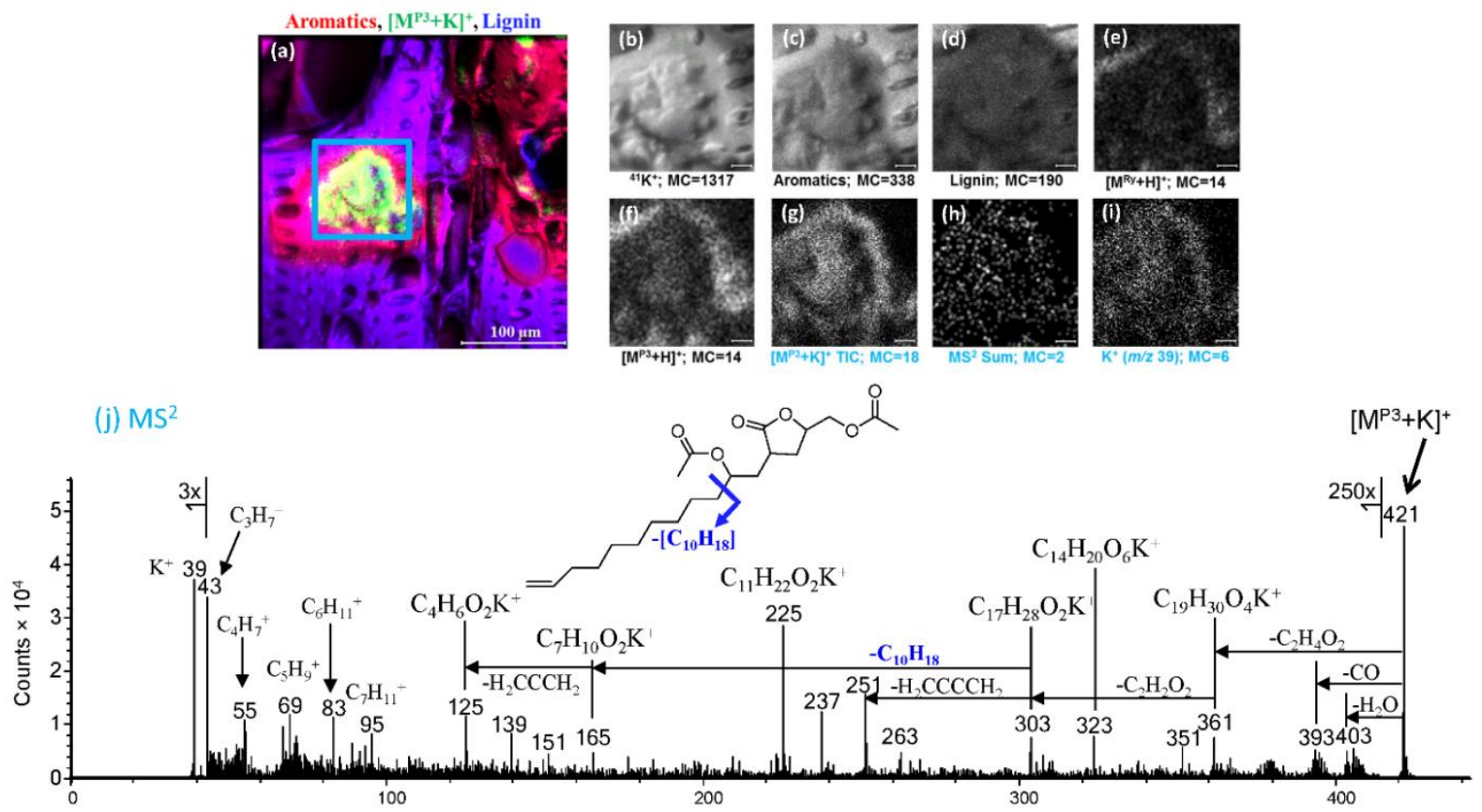
Figure 4: Schematic MALDI MSI workflow. In extension of simple qualitative MSI experiments, qMSI requires calibration against a reference standard on the same slide and deposition of an internal (often isotope-labeled) standard on the sample tissue. Adapted from Schulz, S.; Becker, M.; Groseclose, M. R.; Schadt, S.; Hopf, C. Advanced MALDI mass spectrometry imaging in pharmaceutical research and drug development. Curr. Opin. Biotechnol. 2019, 55, 51-59 (ref 154) under the Creative Commons Attribution 4.0 license (https://creativecommons.org/licenses/by/4.0/).

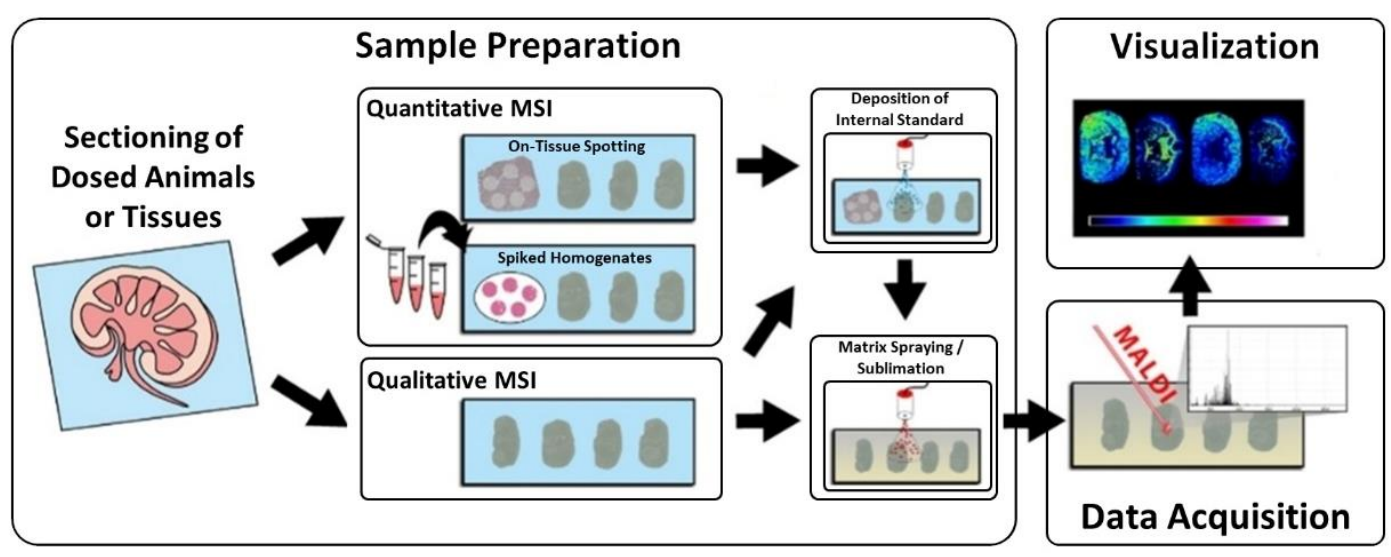


Figure 5: ImzML versatility. Spatial distribution of selected ion images as acquired with different MSI platforms: (a) MALDI-ToF (AMOLF), m/z 14,114; (b) MALDI-QStar (Novartis), m/z 810.6; (c) MALDI-FTICR (PNNL), $m / z$ 810.5983; (d) AP-MALDI-Orbitrap, m/z 848.638; and (e) cluster ToF-SIMS (CNRS), $m / z$ 369.35. All datasets were converted to imzML and displayed in the open-source software (MSIReader) with identical setting for color maps, spatial interpolation, and intensity normalization. (f) H\&E staining of coronal mouse brain section. Colored rectangles indicate analyzed area of measurements shown in $d$ and e. Reprinted by permission from Springer Nature, Römpp, A.; Both, J.P.; Brunelle, A.; Heeren, R.; Laprévote, O.; Prideaux, B.; Seyer, A.; Spengler, B.; Stoeckli, M.; Smith, D., Analytical Bioanalytical Chemistry 2015, 407, 2329-2335 (ref 208). Copyright 2015.

B) MALDI-QStar

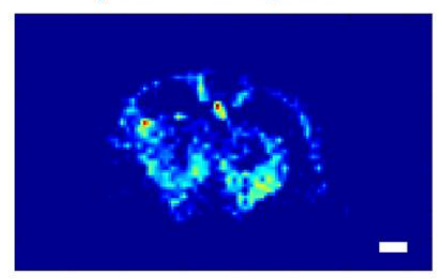

C) MALDI-FTICR

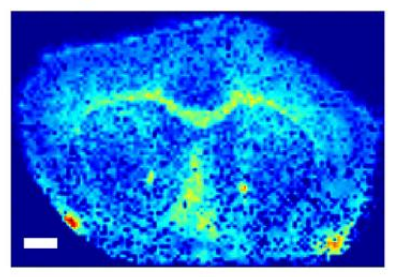

D) AP-MALDI-Orbitrap

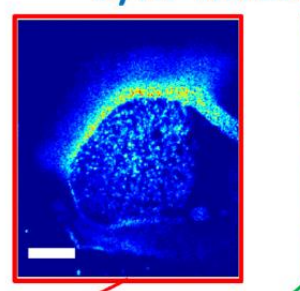

E) Cluster TOF-SIMS

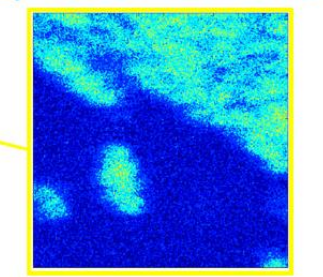


Figure 6: General workflow for multimodal imaging. Red box is defined as the experimental design including the sample preparation as well as the strategies to conduct properly multimodal imaging. The green box corresponds to the sequential data processing related to the processing of individual modality as described previously and whose desire is to achieve the well-established image registration for data correlation. The blue box corresponds to the multimodal data integration in which data integration step is required to achieved advanced data processing. The aim of multimodal imaging is to improve quality of interpretation / discussion of the results (orange box).

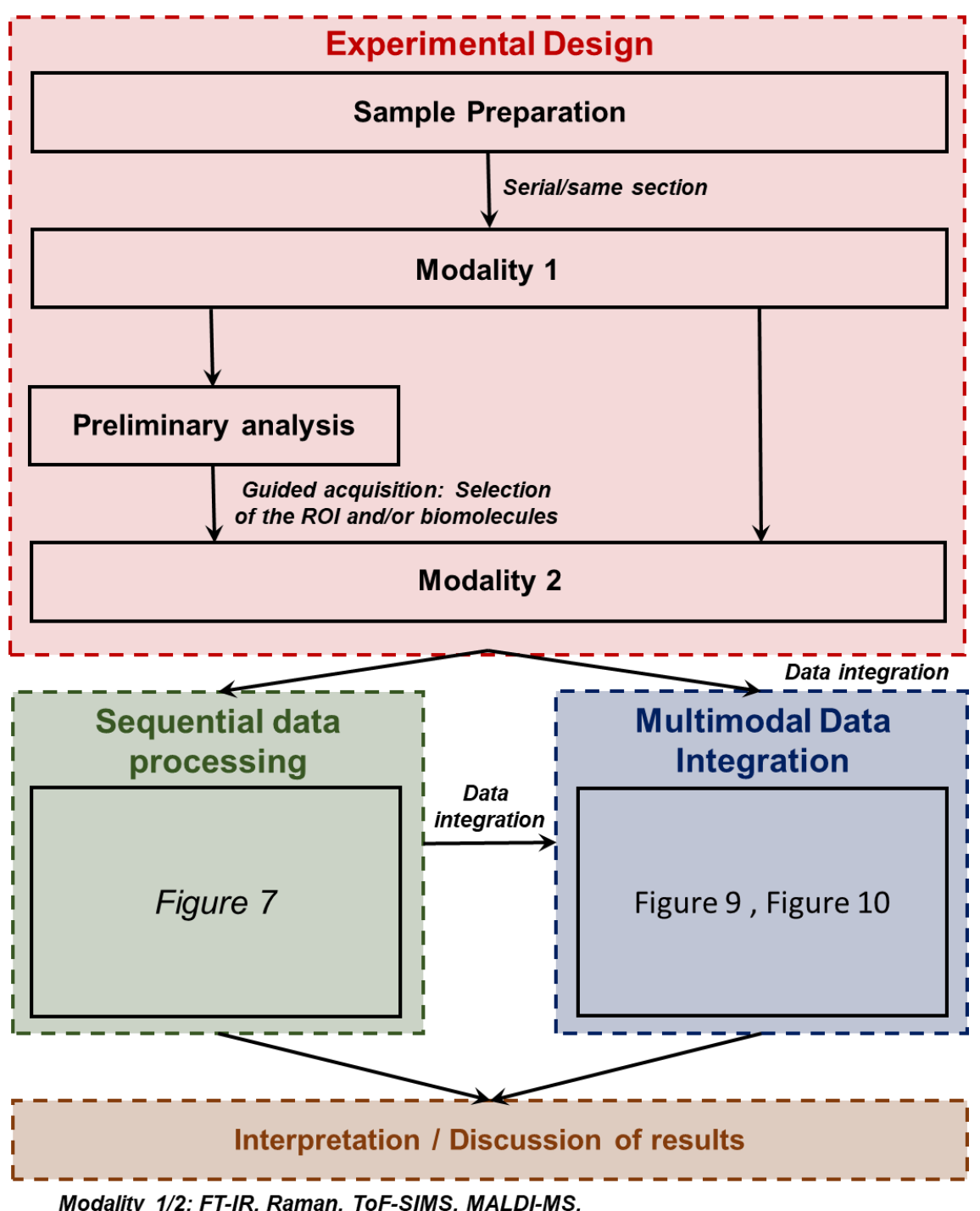


Figure 7: 1) FTIR Segmentation to spatially guide data acquisition by high-resolution MALDI-FTICR-MSI. (a) Multimodal workflow for the consecutive FTIR and MALDI-FTICR-MSI acquisition of a single slide. (b) Based on the FTIR image, segments are calculated based on k-means++ cluster analysis and used for later MSI-guidance. (c) Predefined MALDI-FTICR-MSI acquisition of the granular cell layer significantly decreases data load (97.9\%) and acquisition time (97.8\%). 2) FTIR generated segmentation to guide MALDI MSI profiling (a) FTIR images (absorption band at $2922 \mathrm{~cm}-1$ ) were divided into four segments (I-IV) of maximum data similarity by means of $k$-means++ clustering. The spatial contours reflecting brain morphology were transferred to MS images, in order to be use as masks to guide lipid profiles generation. (b-e) Ten distinct $\mathrm{m} / \mathrm{z}$ features allowing discrimination between healthy and diseased mice were identified using t-test based feature extraction revealing segment and disease-selective changes. For both panels 1) and 2), adapted from Rabe, J.-H.; Sammour, D.A.; Schulz, S.; Munteanu, B.; Ott, M.; Ochs, K.; Hohenberger, P.; Marx, A.; Platten, M.; Opitz, C.A.; Ory, D.S.; Hopf, C. Advanced MALDI mass spectrometry imaging in pharmaceutical research and drug development. Curr. Opin. Biotechnol. 2019, 55, 51-59 (ref 258) under the Creative Commons Attribution 4.0 license (https://creativecommons.org/licenses/by/4.0/).

1) FTIR segmentation to guide MALDI MSI acquisition

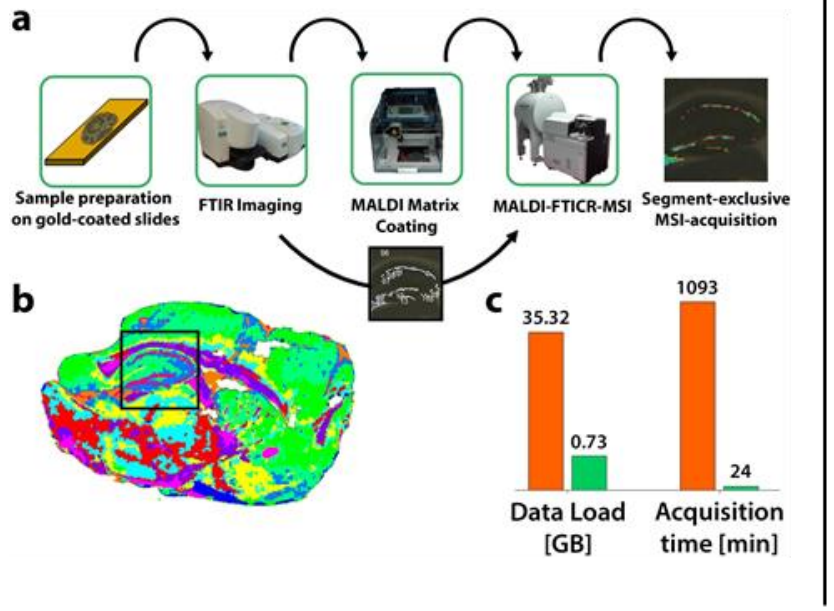

\section{2) FTIR segmentation to guide MALDI MSI processing}

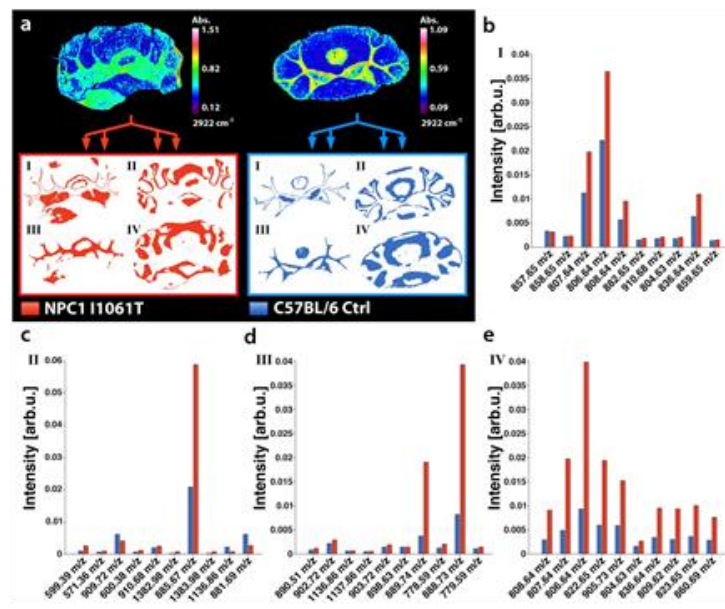


Figure 8: Schematic of some of the challenges of multimodal image registration using a lipid MALDI MSI and H\&E microscopy example. (A) Registration of data with different spatial specificity is a challenge because a representative image must be derived from both datasets. (B) Multi-scale data is common in chemical imaging and the content under a single pixel can be greatly variable.
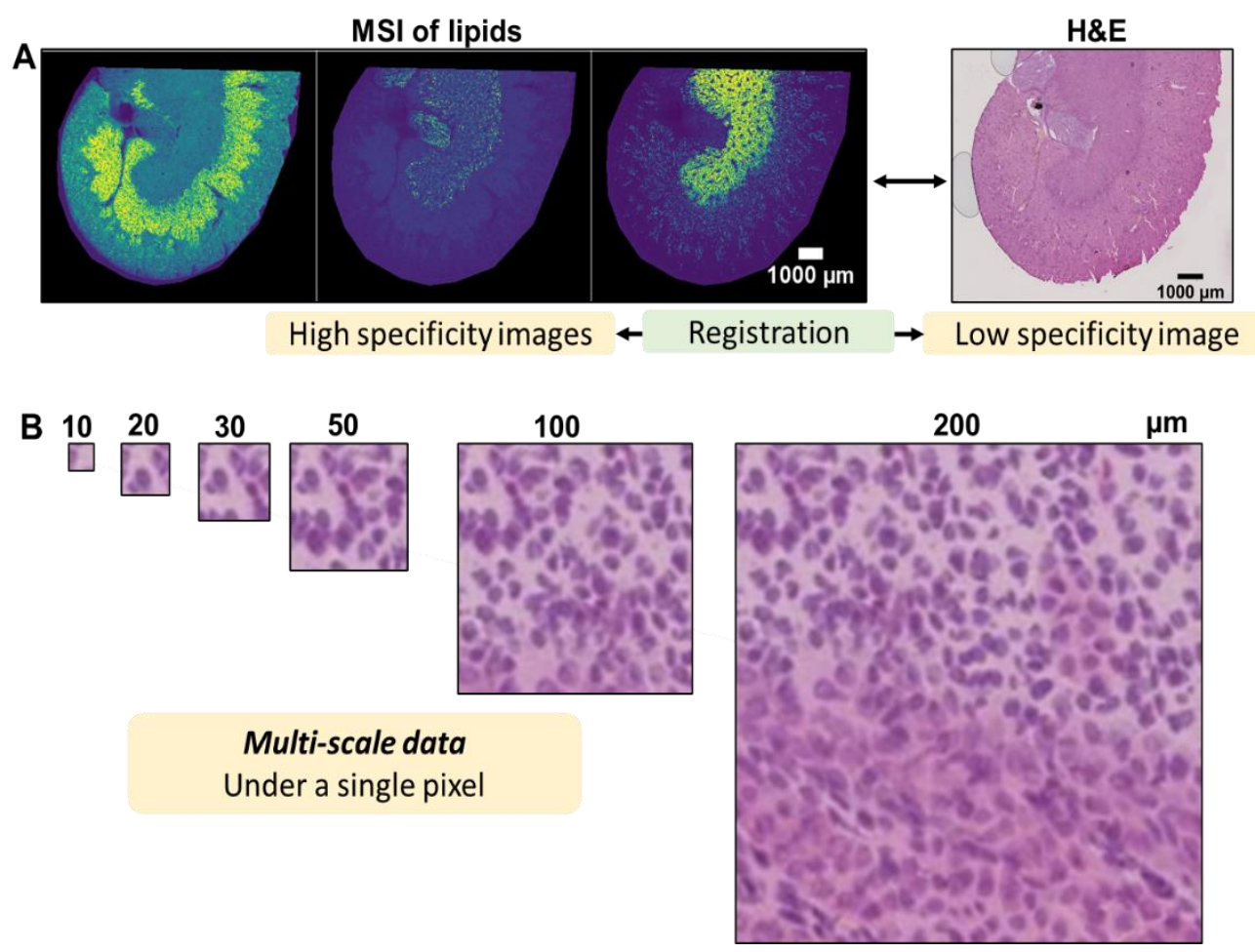

Multi-scale data

Under a single pixel 
Figure 9: 1) Correlation between Raman spectroscopic and MALDI mass spectrometric data. Correlation of two data types after being preprocessed is depicted in yellow (positive values), red (zero), and violet (negative values) colors. 2) Multimodal PCA: first three PCs calculated for MALDI spectra (A), Raman spectra (B), combined Raman-MALDI data (E, F) and their false-color score composites $(C, D, G)$. Red, green, and blue colors indicate the first, second and third PCs, respectively. The PCs composite image of the combined data (G) shows a smoother appearance, and the loadings after data fusion (E, F) are easier to interpret. For both panel 1) and 2), adapted from Ryabchykov, O.; Popp, J.; Bocklitz, T. Fusion of MALDI Spectrometric Imaging and Raman Spectroscopic Data for the Analysis of Biological Samples. Front. Chem. 2018, 6, 1-10 (ref 285) under the Creative Commons Attribution 4.0 license (https://creativecommons.org/licenses/by/4.0/).

1) Multimodal 2D Correlation

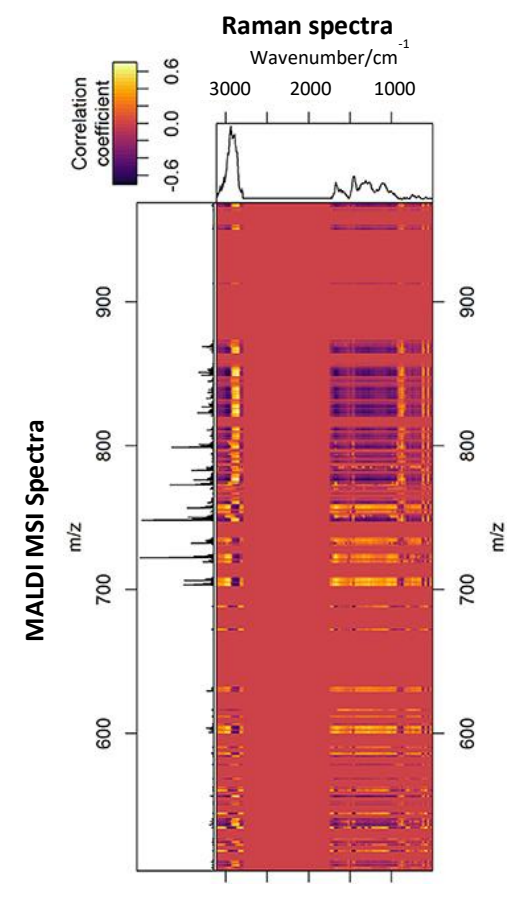

2) Multimodal Principal Component Analysis

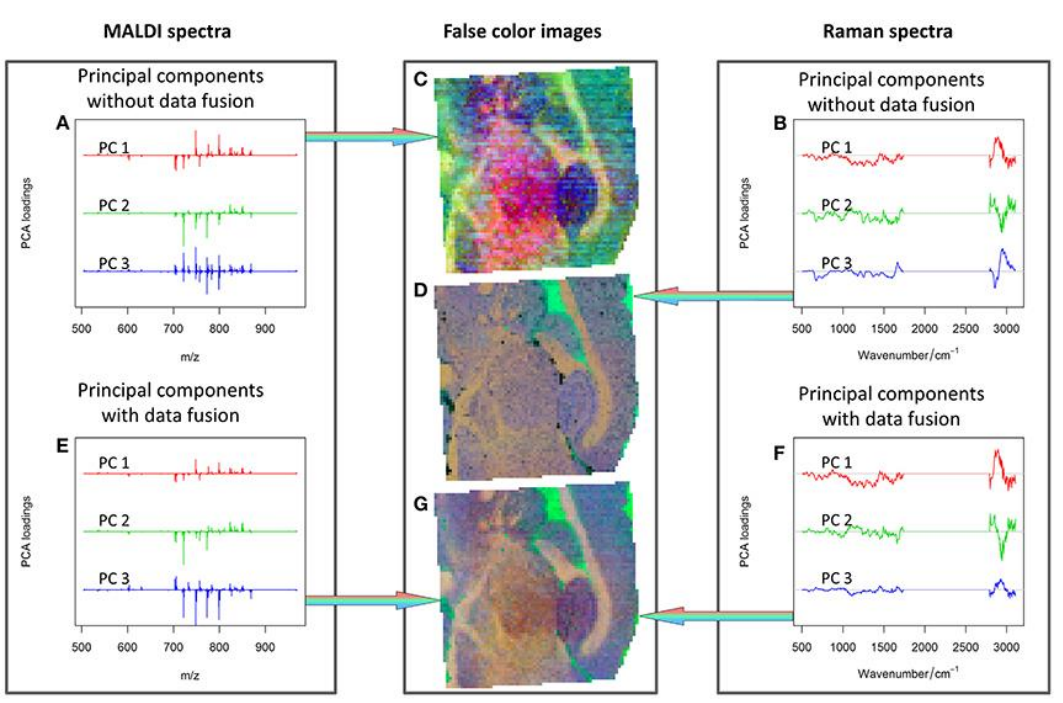


Figure 10: Predictive image sharpening or image fusion. 1) Each data set (represented as a matrix, pixels $x$ $\mathrm{m} / \mathrm{z}$ ) is decomposed by NMF, the factors (spatial factors, pixels $\times k$, where $k$ is the number of factors and spectral factors, $k \times m / z)$ of each data set are then matched and scaled and the fused data is then constructed. Highlighted regions indicate where the displayed example data was extracted from, with the second spatial factor decomposed from the SIMS data, the first spectral factor decomposed from the MALDI data, and the ion image $m / z$ 826.6, both raw and after the fusion process shown. Reproduced from Race, A.M.; Rae, A.; Vorng, J.-L.; Havelund, R.; Dexter, A.; Kumar, N.; Steven, R.T.; Passarelli, M.K.; Tyler, B.J.; Bunch, J.; Gilmore, I. Anal. Chem. 2020, 92, 10979-10988 (ref 275). Copyright 2020 American Chemical Society. 2) a) A MALDI-MSI image of the hippocampus. b) a zoom-in of the MALDI-MSI image. c) a zoom-in of MSI image in panel (a) sharpened with QCL-IR. Adapted from Neumann, E.K.; Comi, T.J.; Spegazzini, N.; Mitchell, J.W.; Rubakhin, S.S.; Gilette, M.U.; Bhargava, R.; Sweedler, J.V. Anal. Chem. 2018, 90, 11572-11580 (ref 259). Copyright 2020 American Chemical Society. 3) A reconstructed image of cholesterol in the hippocampus from an NMF informed workflow from MALDI-MSI and ToF-SIMS. Adapted from Borodinov, N.; Lorenz, M.; King, S.T.; levlev, A.V.; Ovchinnikova, O.S. Toward nanoscale molecular mass spectrometry imaging via physically constrained machine learning on co-registered multimodal data. npj Comput. Mater. 2020, 6, 1-8 (ref 298) under the Creative Commons Attribution 4.0 license (https://creativecommons.org/licenses/by/4.0/).

1) Pictorial description of data fusion process

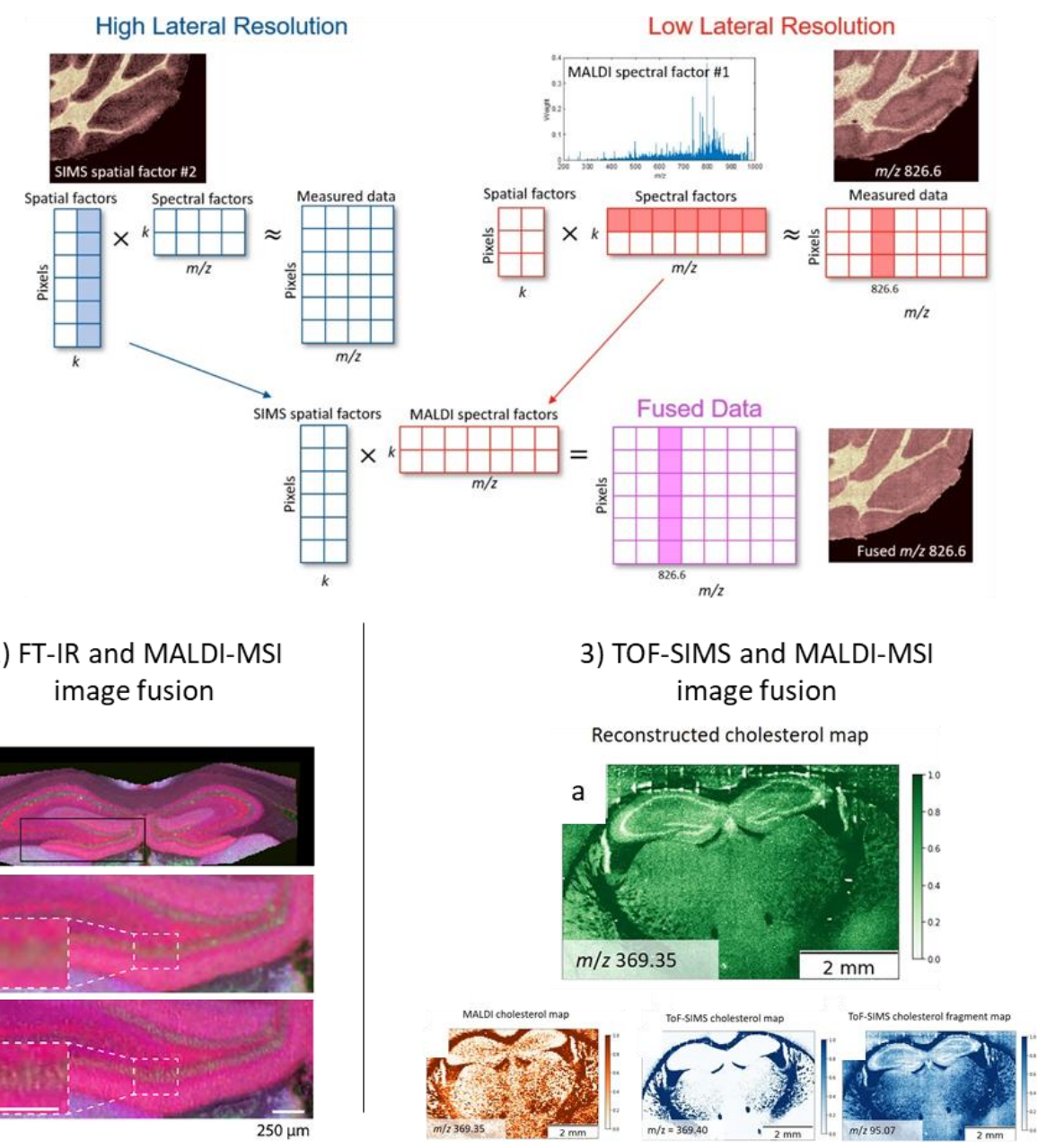


TOC Graphic: Multimodal Imaging, the Next-Gen of Analytical Chemistry Strategy.

Multimodal Imaging:

The Next-Gen of Analytical Chemistry Strategy

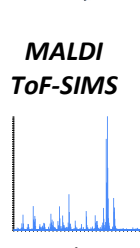

$m / z$

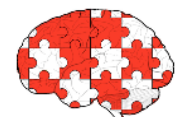

Raman

FTIR

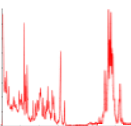

$\mathrm{cm}^{-1}$ 